\title{
PROACTIVE CONTROL OF EMOTIONAL DISTRACTION: AN ERP INVESTIGATION
}

BY

\section{LAURA SARAH KRANZ}

\begin{abstract}
A thesis
submitted to the Victoria University of Wellington

in fulfillment of the requirements for the degree of

Master of Science in
\end{abstract}

Cognitive and Behavioural Neuroscience

Victoria University of Wellington

2015 



\begin{abstract}
According to the Dual Mechanisms of Control (DMC) framework (Braver, 2012) distraction can be controlled either proactively (i.e., before the onset of a distractor) or reactively (i.e., after the onset of a distractor). Research clearly indicates that, when distractors are emotionally neutral, proactive mechanisms are more effective at controlling distraction than reactive mechanisms. However, whether proactive control mechanisms can control irrelevant emotional distractions as effectively as neutral distraction is not known. In the current thesis I examined cognitive control over emotional distraction. In Experiment 1, I tested whether proactive mechanisms can control emotional distraction as effectively as neutral distraction. Participants completed a distraction task. On each trial, they determined whether a centrally presented target letter (embedded amongst a circle of 'o's) was an ' $\mathrm{X}$ ' or an ' $\mathrm{N}$ ', while ignoring peripheral distractors (negative, neutral, or positive images).

Distractors were presented on either a low proportion (25\%) or a high proportion $(75 \%)$ of trials, to evoke reactive and proactive cognitive control strategies, respectively. Emotional images (both positive and negative) produced more distraction than neutral images in the low distractor frequency (i.e., reactive control) condition. Critically, emotional distraction was almost abolished in the high distractor frequency condition; emotional images were only slightly more distracting than neutral images, suggesting that proactive mechanisms can control emotional distraction almost as effectively as neutral distraction. In Experiment 2, I replicated and extended Experiment 1. ERPs were recorded while participants completed the distraction task. An early index (the early posterior negativity; EPN) and a late index (the late positive potential; LPP) of emotional processing were examined to investigate the mechanisms by which proactive control minimises emotional distraction. The behavioural results of Experiment 2 replicated Experiment 1, providing further support for the hypothesis that proactive mechanisms can control emotional distractions as effectively as neutral distractions. While proactive control was found to eliminate early emotional processing of positive distractors, it paradoxically did not attenuate late emotional processing of positive distractors. On the other hand, proactive control eliminated late emotional processing of negative distractors. However, the early index of emotional processing was not a reliable index of negative distractor processing under either reactive or proactive conditions. Taken together, my findings show that proactive mechanisms can effectively control emotional distraction, but do not clearly establish the mechanisms by which this occurs.
\end{abstract}




\section{Acknowledgements}

First, I would like to thank my supervisor, Dr. Gina Grimshaw. You have been an amazing supervisor and mentor. I feel like I have learnt so much from you since I joined the lab. You have always been supportive, provided guidance when I needed it, and have inspired me to be the best scientist I can be. I also want to thank you for fostering a wonderful lab environment to conduct science in. Thank you for the things I have listed, and for the other giant list of things that I should thank you for which won't fit in this acknowledgements section. I feel lucky to have had you as my supervisor. I also owe thanks to Dr. David Carmel who has provided valuable input into this project.

I also owe a huge thanks to my family. Mum and Dad, thanks for being the most supportive parents I could ask for. You are both so encouraging and have always helped me to follow my passions. I cannot imagine making it to this point without you both. Joel and Sophie, thanks for being awesome siblings - I know you are both there for me when I need it. I also want to thank my Gran. Gran, you are truly a role model to me and I am lucky to have you in my life. You always take an active interest in what I do, and support and inspire me. I appreciate all of your support and guidance (and also your help on crossword puzzles).

I would also like to thank all the members of the CANlab for being so supportive, always willing to help out, and just a really fun bunch of people to do science with. I would like to particularly thank Hazel and Rosie. Hazel - you are the best desk-buddy and flat mate, and your support and encouragement has made my Masters journey that much easier (and more enjoyable). Rosie - thank you for all of your support and help. And thanks for being my adventure buddy and making me enjoy extracurricular activities during my Masters. With it all over and done with I hope we can spend more time diving!

I also have incredibly supportive friends outside of Uni. I would like to particularly thank Vicky, Hayley and Liora. You are all wonderful friends, and have all helped get me through my Masters. A special thanks to Vic for always listening to me moan (no matter what time of day it was) when I needed it! I am so lucky to have such good friends in my life.

One of the people I owe the biggest thanks to is my boyfriend Michael. You have been the most supportive, helpful and wonderful person ever throughout this whole process. Without you I don't know how I would have reached this point. You made me take breaks when I needed them and helped motivate me to work when I couldn't motivate myself. You have also put up with watching Friends on a constant loop. Thank you for everything. I cannot express how lucky I feel to have had you by my side. 
Finally, I was lucky enough to receive a Victoria Master's (by thesis) Scholarship, and a Freemasons Postgraduate Scholarship. I truly appreciate this financial support, and it has made a huge difference to my Masters experience. 


\section{Table of Contents}

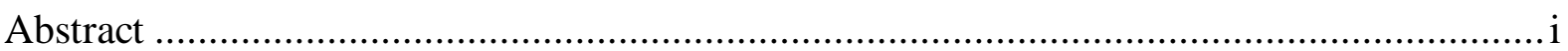

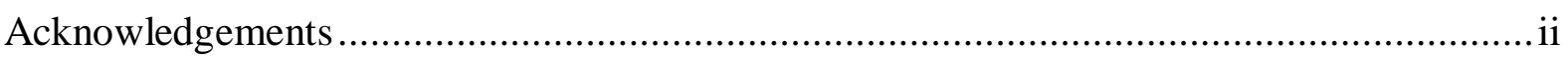

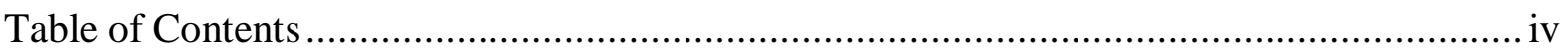

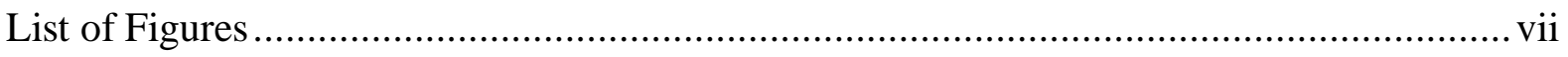

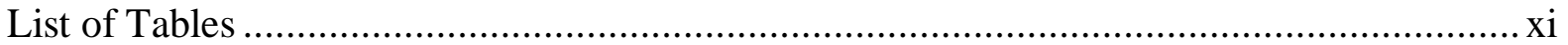

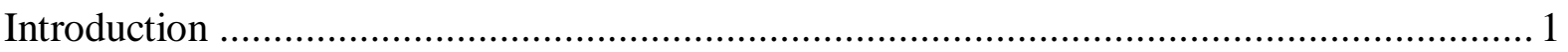

Controlling Distraction: Proactive and Reactive Cognitive Control................................. 1

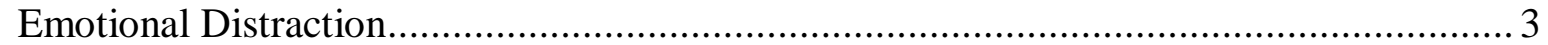

Task-irrelevant Emotional Distraction ................................................................... 3

Evidence against Task-Irrelevant Emotional Distraction. ............................................ 3

Evidence for Task-Irrelevant Emotional Distraction. ................................................ 5

Control of Emotional Distraction ............................................................................... 7

Empirical Evidence: Can Emotional Stimuli be Effectively Controlled? ......................... 10

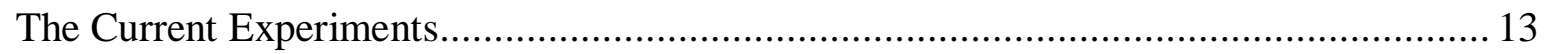

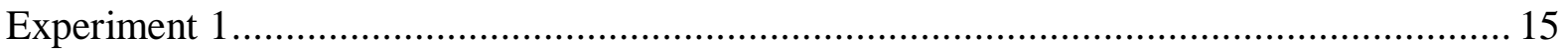

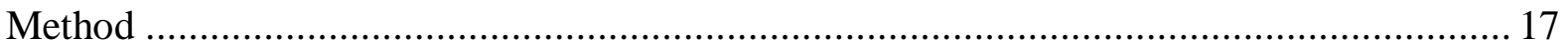

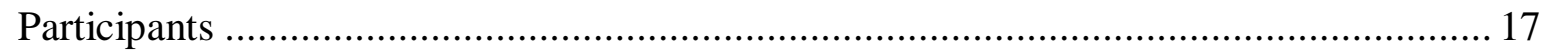

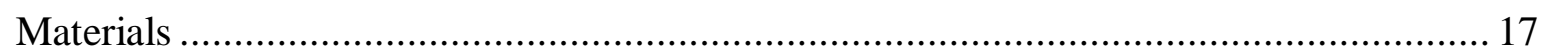

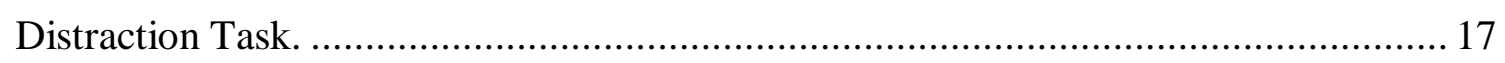

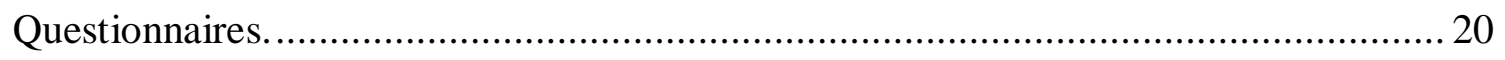

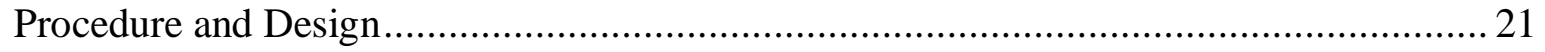

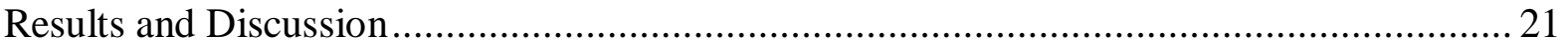

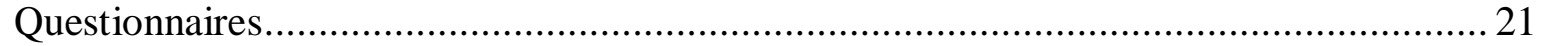

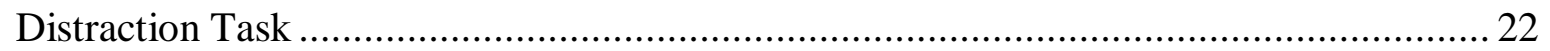

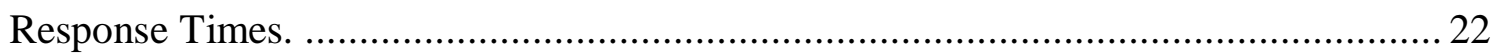

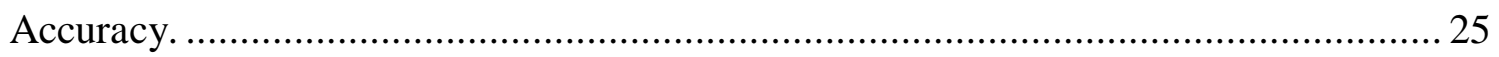

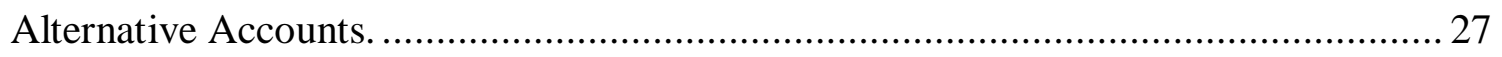

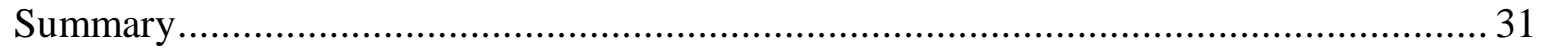

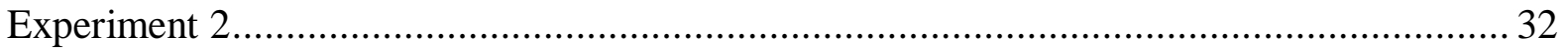

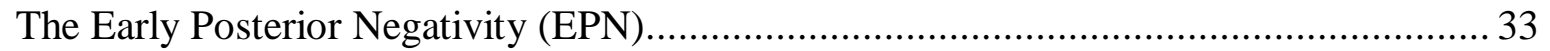




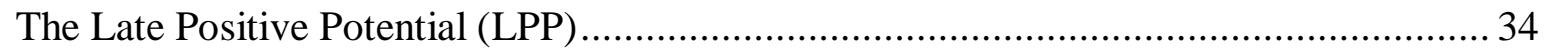

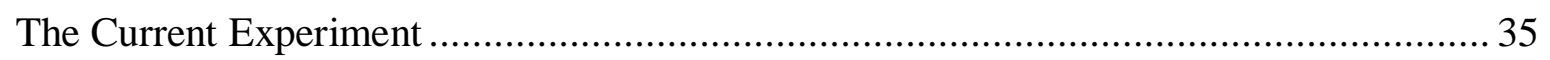

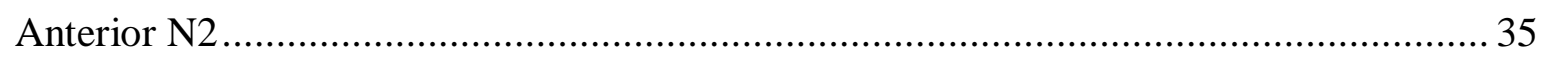

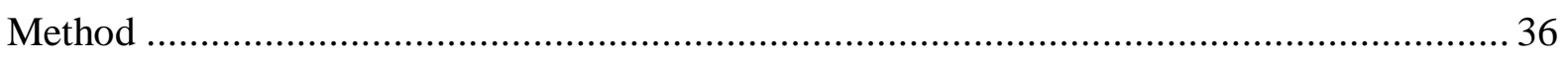

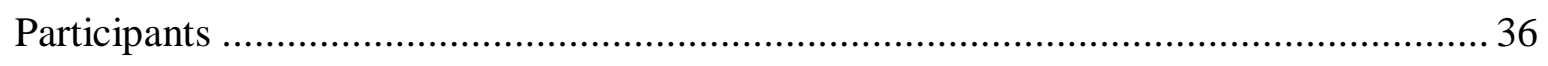

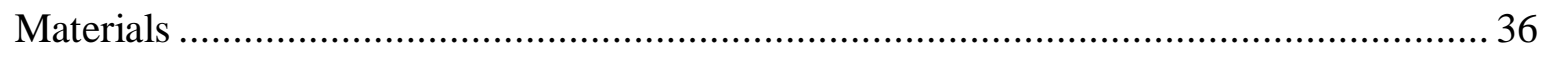

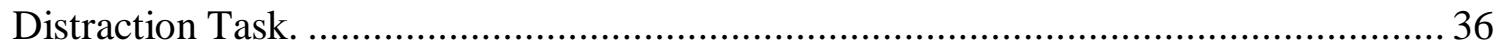

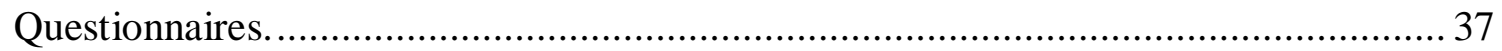

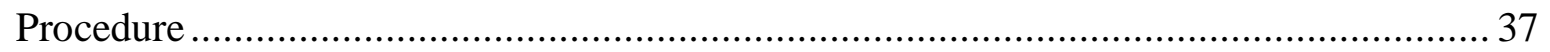

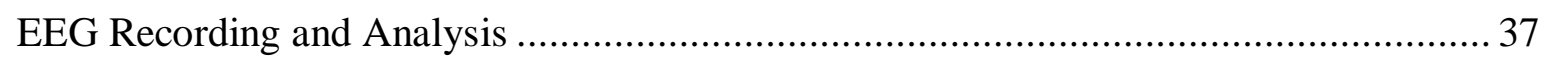

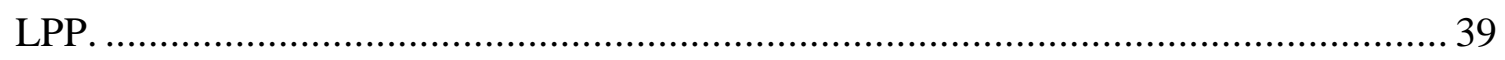

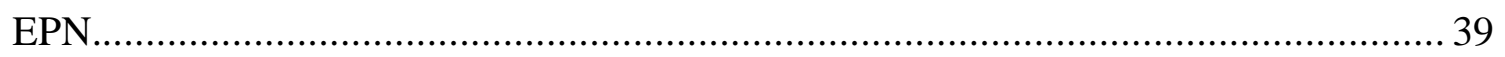

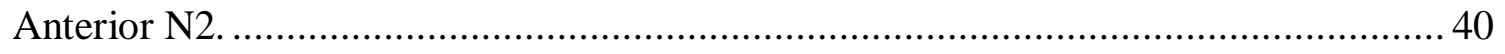

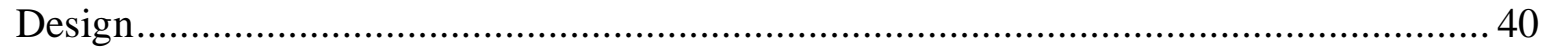

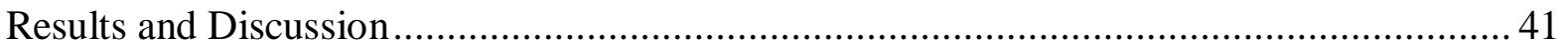

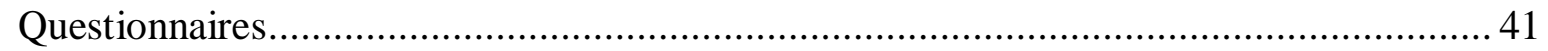

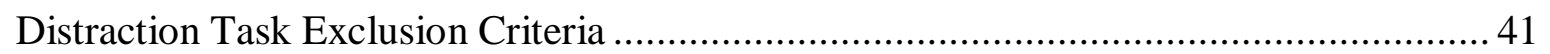

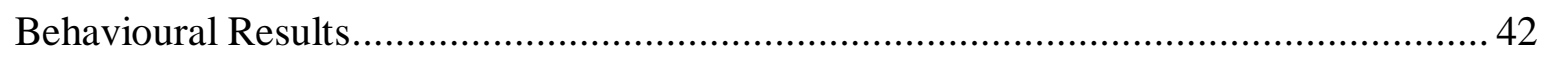

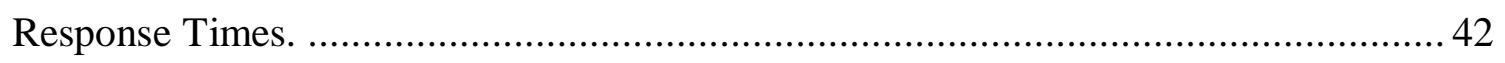

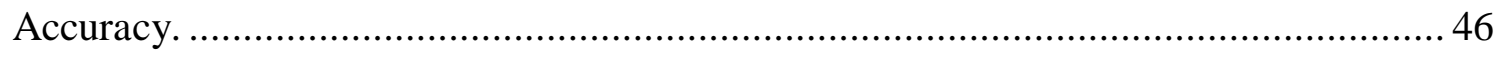

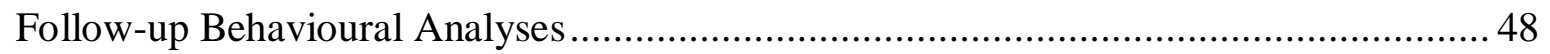

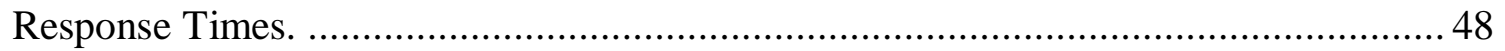

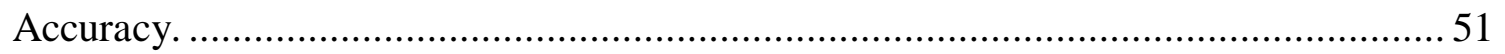

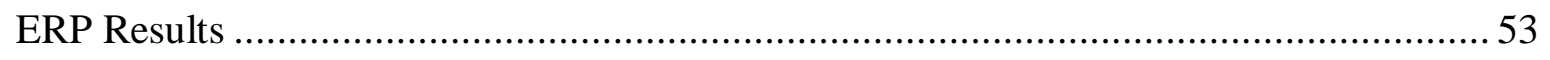

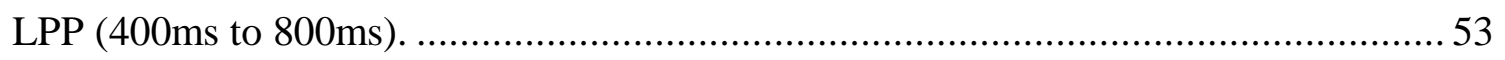

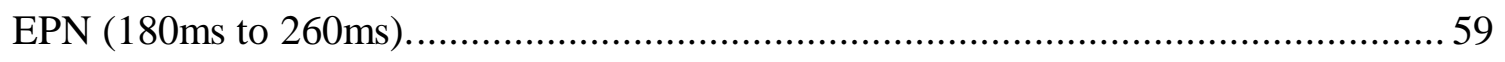

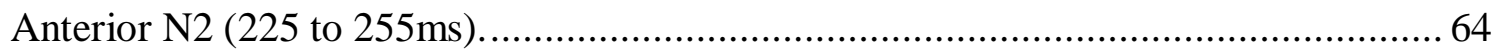

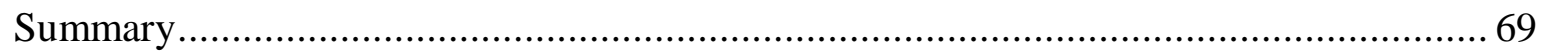

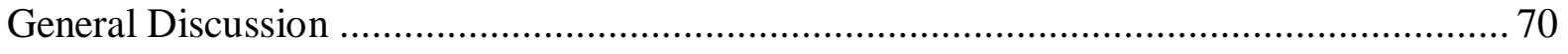

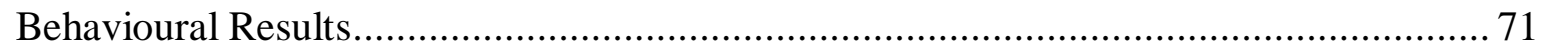

Differences in Behavioural Results across Experiments. .......................................... 73

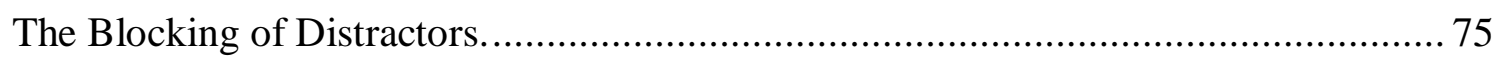

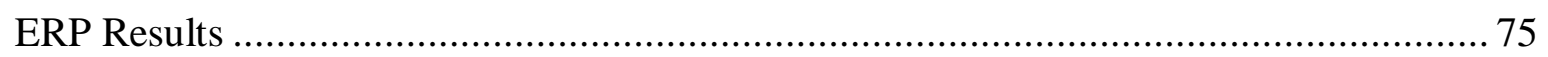




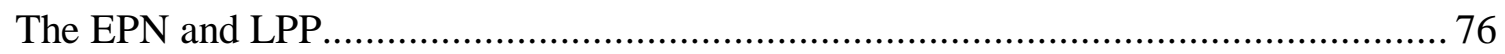

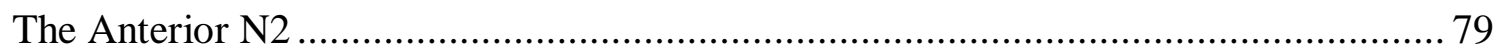

Similarities across ERP Components: The Positivity Bias...................................... 81

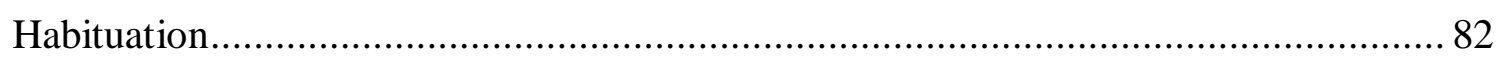

Future Directions: Extending our Understanding of how Proactive Mechanisms Control

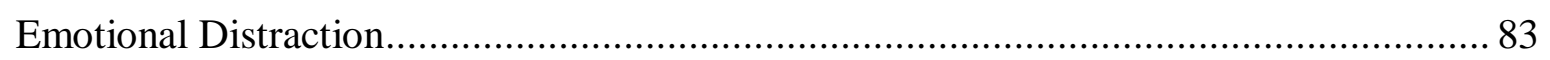

Finding a reliable index of negative distractor processing. .................................. 83

Is anticipating the valence or emotionality of an upcoming distractor necessary to engage proactive control mechanisms?

Is proactive control of neutral distraction easier than proactive control of emotional

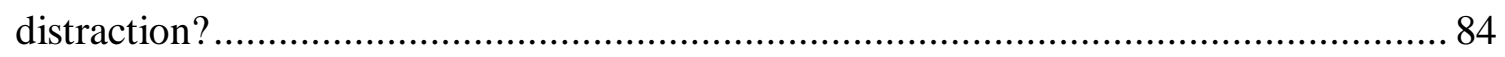

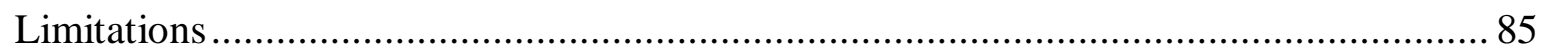

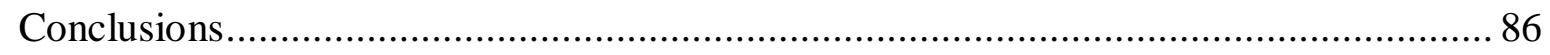

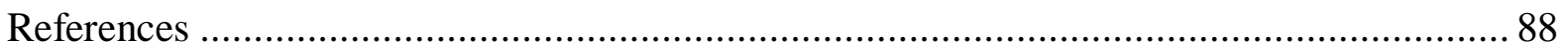

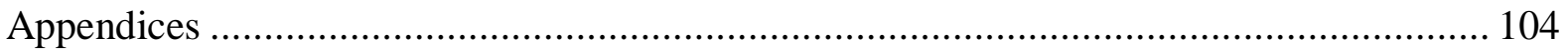




\section{List of Figures}

Figure 1. An example of what experimental data might look like if emotional attention is independent of top-down control..

Figure 2. An example of what experimental data might look like if emotional attention can be modulated by top-down control..

Figure 3. An example of a typical trial procedure in the distraction task.

Figure 4. Schematic representation of the blocking procedure and counterbalances for the distraction task. Within each block, participants saw a mixture of distractor-present and distractor-absent trials, as determined by their distractor probability condition.

Figure 5. Mean RTs on the distraction task by distractor frequency, emotionality and distractor presence in Experiment 1. Pronounced emotional (compared to neutral) distraction was observed in the low distractor frequency condition. In the high distractor frequency condition emotional distraction was markedly reduced.

Figure 6. Mean accuracy (\%) on the distraction task. Accuracy was lower on negative distractor-present, than negative distractor-absent trials in the low distractor frequency condition in Experiment 1. In the high distractor frequency condition accuracy was higher on distractor-present, than distractor-absent negative trials. . 27

Figure 7. Mean RTs on the distraction task for emotional distractors, using only the first 24 distractor-present/distractor-absent trials, by distractor frequency condition in Experiment 1.

Figure 8. Mean distraction index (RT on distractor-present - RT on distractor-absent trials) by experiment half in Experiment 1 .....

Figure 9. Scalp location of the electrodes of interest.

Figure 10. Mean RTs on the distraction task, for participants who met the behavioural inclusion criteria, by distractor frequency, valence and distractor presence in Experiment 2.

Figure 11. Mean distraction Index (RT on distractor-present trials - RT on distractor-absent trials), for participants who met the behavioural inclusion criteria, by distractor frequency and valence in Experiment 2. Pronounced emotional (compared to neutral) distraction was observed in the low distractor frequency condition. No difference between emotional and neutral distraction was observed in the high distractor frequency condition. 
Figure 12. Mean accuracy (\%) on the distraction task, for participants who met the behavioural inclusion criteria, by distractor frequency, valence and distractor presence in Experiment 2.

Figure 13. Mean RTs on the distraction task, for participants who met the ERP inclusion criteria, by distractor frequency, valence and distractor presence in Experiment 2.

Figure 14. Mean distraction Index (RT on distractor-present trials - RT on distractor-absent trials), for participants who met the ERP inclusion criteria, by distractor frequency and valence in Experiment 2. Pronounced positive, but not negative, (compared to neutral) distraction was observed in the low distractor frequency condition. No difference between emotional and neutral distraction was observed in the high distractor frequency condition.

Figure 15. Mean accuracy (\%) on the distraction task, for participants who met the ERP inclusion criteria, by distractor frequency, valence and distractor presence in Experiment 2.

Figure 16. LPP amplitudes in the low distractor frequency condition collapsed across electrodes (PZ, CP3, CP4, P3, P4) by valence. Positive and negative images elicited a larger (i.e., more positive) LPP than neutral images. There was no difference in LPP amplitudes elicited by positive and negative images. 56

Figure 17. LPP amplitudes in the high distractor frequency condition by valence. Positive images elicited a larger (i.e., more positive) LPP than neutral and negative images. There was no difference in LPP amplitudes elicited by neutral and negative images.

Figure 18. (A) Difference waves (positive - neutral) for LPPs elicited by positive and neutral images, collapsed across electrodes (PZ, CP3, CP4, P3, P4), by distractor frequency condition. More positive values reflect a larger LPP for positive, compared to neutral, images. Scalp distribution for the positive-neutral difference are depicted for the low distractor frequency condition (B) and high distractor frequency condition (C).

Figure 19. (A) Difference waves (negative - neutral) for LPPs elicited by negative and neutral images, collapsed across electrodes (PZ, CP3, CP4, P3, P4), by distractor frequency condition. More positive values reflect a larger LPP for negative, compared to neutral, images. Scalp distribution for the negative-neutral difference 
are depicted for the low distractor frequency condition (B) and high distractor frequency condition $(\mathrm{C})$.

Figure 20. EPN amplitudes in the low distractor frequency condition collapsed across electrodes $(\mathrm{O} 1, \mathrm{OZ}, \mathrm{O} 2)$, by valence. Positive images elicited a larger (i.e., more negative) EPN than neutral and negative images. There was no difference in EPN amplitudes elicited by neutral and negative images.

Figure 21. EPN amplitudes in the high distractor frequency condition collapsed across electrodes $(\mathrm{O} 1, \mathrm{OZ}, \mathrm{O} 2)$, by valence. There was no difference in EPN amplitudes elicited by neutral, positive and negative images.

Figure 22. (A) Difference waves (positive - neutral) for EPNs elicited by positive and neutral images, collapsed across electrodes $(\mathrm{O} 1, \mathrm{OZ}, \mathrm{O} 2)$, by distractor frequency condition. More negative values reflect a larger EPN for positive, compared to neutral, images. Scalp distribution for the positive-neutral difference are depicted for the low distractor frequency condition (B) and high distractor frequency condition $(\mathrm{C})$.

Figure 23. (A) Difference waves (negative - neutral) for EPNs elicited by negative and neutral images, collapsed across electrodes $(\mathrm{O} 1, \mathrm{OZ}, \mathrm{O} 2)$, by distractor frequency condition. More negative values reflect a larger EPN for negative, compared to neutral, images. Scalp distribution for the negative-neutral difference are depicted for the low distractor frequency condition (B) and high distractor frequency condition (C).

Figure 24. Anterior N2 amplitudes in the low distractor frequency condition, collapsed across electrodes (F3, FZ, F4), by valence. Neutral images elicited a larger (i.e., more negative) N2 than positive images. Negative images elicited a marginally larger anterior N2 than positive images. There was no difference in anterior N2 amplitudes elicited by neutral and negative images.

Figure 25. Anterior N2 amplitudes for in the high distractor frequency condition, collapsed across electrodes (F3, FZ, F4), by valence. There was no difference in anterior N2 amplitudes elicited by neutral, positive and negative images.

Figure 26. (A) Difference waves (positive - neutral) for anterior N2s elicited by positive and neutral images, collapsed across electrodes (F3, FZ, F4), by distractor frequency condition. More positive values reflect a smaller anterior N2 for positive, compared to neutral, images. Scalp distribution for the positive-neutral difference 
are depicted for the low distractor frequency condition (B) and high distractor frequency condition $(\mathrm{C})$.

Figure 27. (A) Difference waves (negative - neutral) for anterior N2s elicited by negative and neutral images, collapsed across electrodes (F3, FZ, F4), by distractor frequency condition. More positive values reflect a smaller anterior N2 for negative, compared to neutral, images. Scalp distribution for the negative-neutral difference are depicted for the low distractor frequency condition (B) and high distractor frequency condition (C). 


\section{List of Tables}

Table 1. Mean (SD) valence and arousal ratings for the picture stimuli used in the distraction task.

Table 2. Mean (SD) questionnaire scores by distractor frequency condition in Experiment 1

Table 3. Mean (SD) RTs (ms) for the distraction task by distractor frequency, valence, emotionality, and distractor presence in Experiment 1

Table 4. Mean (SD) accuracy (\%) for the distraction task by distractor frequency, valence, emotionality, and distractor presence in Experiment 1.

Table 5. Mean (SD) RTs (ms) for the first 24 distractor-present and distractor-absent trials in blocks with emotional distractors by distractor frequency, and distractor presence in Experiment 1

Table 6. Mean (SD) distraction indices (RT on distractor-present trials - RT on distractorabsent trials) by distractor frequency, experiment half, valence order, and emotionality in Experiment 1.

Table 7. Mean (SD) questionnaire scores by distractor frequency in Experiment 2

Table 8. Mean (SD) RTs (ms) for the distraction task, for participants who met the behavioural inclusion criteria, by distractor frequency, valence and distractor presence in Experiment 2

Table 9. Mean (SD) accuracy (\%) for the distraction task, for participants who met the behavioural inclusion criteria, by distractor frequency, valence and distractor presence in Experiment 2

Table 10. Mean (SD) RTs (ms) for the distraction task, for participants who met the behavioural inclusion criteria, by distractor frequency, valence and distractor presence in Experiment 2 .....

Table 11. Mean (SD) accuracy (\%) for the distraction task, for participants who met the behavioural inclusion criteria, by distractor frequency, valence and distractor presence

Table 12. Mean (SD) LPP amplitudes ( $\mu \mathrm{V})$, collapsed across electrode, by distractor frequency and valence

Table 13. Mean (SD) EPN amplitudes $(\mu \mathrm{V})$, collapsed across electrode, by distractor frequency and valence

Table 14. Mean (SD) anterior N2 amplitudes $(\mu \mathrm{V})$, collapsed across electrode, by distractor frequency and valence 


\section{Proactive Control of Emotional Distraction: An ERP Investigation}

The visual world around us is complex. Attentional and perceptual systems do not process all of the visual information in the environment to the same degree (Corbetta $\&$ Shulman, 2002; Desimone \& Duncan, 1995). Therefore, a subset of information must get selected for further processing. Emotional information tends to be selected for further processing at the expense of neutral information - that is, it is preferentially attended to and receives prioritised perceptual processing (Pourtois, Schettino \& Vuilleumier, 2013; Vuilleumier, 2005). From an evolutionary perspective this makes sense because emotional stimuli signal information potentially important for our survival such as the possibility of threat or reward, and thus facilitate adaptive behaviours (LeDoux, 1996). However, emotional stimuli are often irrelevant to our primary goals. In order to achieve our goals, attention to irrelevant emotional stimuli must be controlled. Limited research has investigated whether or how we are able to control attention to emotional distractors. Broadly, research suggests that neutral distraction can be effectively controlled when it is anticipated (Braver, 2012; Chao, 2011, Lee \& Chao, 2012; Munneke, Van der Stigchel \& Theeuwes, 2008; Padmala \& Pessoa, 2011; Van der Stigchel \& Theeuwes, 2006). When distractors are anticipated, proactive control processes can be recruited to minimise distraction before the onset of the distractor (Braver, 2012). However, whether irrelevant emotional distractions can be similarly controlled is not clear. Understanding how we control attention to emotional distractors is important, not only because they are prevalent in everyday life, but also because failure to control emotional distraction is associated with a range of clinical disorders - e.g., depression and anxiety (see Williams, Mathews \& MacLeod, 1996), and addiction (see Field \& Cox, 2008).

In this thesis I present two experiments that examined control of irrelevant emotional distractions. In Experiment 1, I determined whether attention to emotional distractors can be as effectively controlled as attention to neutral distractors when they are anticipated. In Experiment 2, I used event related potentials (ERPs) to assess the time-course of emotional processing of distractors, both when anticipated and not anticipated. This was done to examine the mechanisms by which cognitive control reduces distraction from irrelevant emotional information.

\section{Controlling Distraction: Proactive and Reactive Cognitive Control}

Achieving day-to-day goals often depends on our ability to control distraction by irrelevant information. To control distraction, and focus on goal-relevant information, cognitive control processes are recruited. Cognitive control comprises top-down processes 
that regulate attention, perceptual processing, and behaviour to facilitate our current goals (Braver, 2012). According to the Dual Mechanisms of Control framework (DMC; Braver, 2012; Braver, Paxton, Locke \& Barch, 2009) there are two mechanisms by which cognitive control is exerted in order to prioritise goal-relevant information and reduce distraction from irrelevant information: proactive control and reactive control. Proactive control is engaged before the onset of a distractor, and directs attentional resources in a top-down goal-driven manner away from expected distractors, and/or towards goal-relevant information. Processing systems are proactively altered to devote resources to relevant information and minimise processing of irrelevant information. On the other hand, reactive control is triggered after the onset of a distractor and allows us to direct our attention away from potential distractors to re-focus on goal-relevant information.

The relative reliance on proactive or reactive control in a given situation can be seen as the outcome of a cost-benefit analysis; while proactive control is more effective at controlling distraction than reactive control, it consumes more cognitive resources as it relies upon the active maintenance of a goal (Braver, 2012). If the task at hand is particularly important, or distractors are frequent, it may be worth the effort to maintain proactive control. According to the DMC framework (Braver, 2012), individual differences may also affect the relative use of proactive or reactive cognitive control. In fact, some researchers argue that a number of psychopathologies are underpinned by failure to successfully exert cognitive (likely proactive) control over distraction (Braver, 2012; Burgess et al., 2010; Edwards, Barch \& Braver, 2010; Eysenck, Derakshan, Santos \& Calvo, 2007; Mayberg, 1997; West, Choi \& Travers, 2010).

For the most part, cognitive control over distraction has been examined when distractors are neutral. However, many of the distractions we experience in everyday life have emotional value attached to them (e.g., attractive classmates, drivers with road-rage, spiders, etc.). Research clearly indicates that neutral distraction is more effectively controlled by proactive than reactive strategies (e.g., Chao, 2011, Lee \& Chao, 2012; Munneke et al., 2008; Padmala \& Pessoa, 2011; Van der Stigchel \& Theeuwes, 2006). However, it is unclear whether proactive mechanisms are able to control irrelevant emotional distractions as effectively. Emotional stimuli are argued to be particularly potent distractors (Okon-Singer, Lichtenstein-Vidne \& Cohen, 2013; Pourtois et al., 2013; Yiend, 2010) and so may place different demands on cognitive control. 


\section{Emotional Distraction}

Emotional stimuli are important and relevant for survival (LeDoux, 1996). As such, it makes sense that irrelevant emotional stimuli distract us from primary tasks to a greater extent than irrelevant neutral stimuli. Indeed, a snake that slithers past you in the grass is not relevant to your goal of mowing the lawns, but is relevant to your goal of surviving. A number of empirical studies have examined whether emotional distractors are more potent than neutral distractors. However, very few studies use paradigms that examine truly taskirrelevant emotional distraction. In many cases emotion is an irrelevant property of an attended object either because it is the target (as, for example, in the emotional Stroop task; see Phaf \& Kan, 2007 for a meta-analysis; see Williams et al., 1996 for a review), or because it could be the target (as, for example, in variants of the visual search task in which the emotionality of distractors in the array is manipulated; e.g., Hodsoll, Viding \& Lavie, 2011; Lipp \& Waters, 2007; Miltner, Krieschel, Hecht, Trippe \& Weiss, 2004). In other studies, emotional distractors are distinct from the target stimulus, but are presented in attended spatial locations - either because they are presented in the same location as targets (e.g., Attar \& Müller, 2012; Haas, Omura, Constable \& Canli, 2006, 2007; Sussman, Heller, Miller \& Mohanty, 2013), or at fixation (e.g., Augst, Kleinsorge \& Kunde, 2014; Erthal et al., 2005; Fernandes, Koji, Dixon \& Aquino, 2011) which is an inherently attended location (see Beck $\&$ Lavie, 2005). When distractors are relevant, or share properties with relevant information (i.e., are a property of an attended object, or are presented in an attended location), there is robust evidence that emotional information is more distracting than neutral information. However, whether entirely task-irrelevant distractors are more potent than neutral distractors is more controversial.

\section{Task-irrelevant Emotional Distraction}

Few studies have examined emotional distraction under truly task-irrelevant conditions - that is, when emotional distractors are non-attended objects, presented in nonattended locations. From the limited research that has been conducted, evidence for pronounced emotional (compared to neutral) distraction is mixed.

\section{Evidence against Task-Irrelevant Emotional Distraction.}

Three studies have found that irrelevant emotional stimuli are no more potent than irrelevant neutral stimuli (Barratt \& Bundesen, 2012; Lichtenstein-Vidne, Henik \& Safadi, 2012; Okon-singer, Tzelgov \& Henik, 2007). Lichtenstein-Vidne and colleagues (2012) used an emotional flanker task to examine distraction. In a typical flanker task, participants perform a primary task at fixation, while ignoring peripheral distractors (Eriksen \& Eriksen, 
1974). Flankers that are somehow inconsistent with the target stimulus impair performance as long as the primary task is perceptually simple (that is, a 'low' perceptual load task). If the primary task is perceptually complex (a 'high' perceptual load task), flanker effects can be eliminated (Lavie, 1995). In their first experiment, Lichtenstein-Vidne et al. (2012) had participants complete a flanker task under low perceptual load; they detected the location of neutral pictures presented directly above or below fixation (i.e., in the 'middle' column of the screen). On every trial, irrelevant flankers (positive, negative or neutral images) were presented in the two columns either side of fixation (spatial areas where targets never appeared). They found that the valence of the distractor did not affect response times (RTs) for location judgment - i.e., no evidence for an emotional distraction effect. Experiment 2 used the same design as Experiment 1, but emotion was made relevant by changing the targets to emotional pictures - if participants are monitoring for emotional targets, they may be sensitive to emotion in the distractors. In Experiment 2, a negative emotional distraction effect was observed; location judgment was slower when distractors were negative relative to neutral or positive. Taken together, the results from this study suggest that emotional distraction is contingent on distractors sharing emotional features of targets.

Similarly, Barratt and Bundesen (2012) examined whether manipulating the relevance of emotion affected distraction in an emotional flanker task. In Experiment 1, participants determined whether a central face was positive or negative (meaning emotion was relevant), while ignoring distracting flankers on either side of the target. Distractors were presented on $75 \%$ of trials, and comprised positive (happy), negative (angry or sad) or neutral faces. Evidence for a negative emotional distraction effect was found; target discrimination was slower in the presence of negative, than positive, distractors. They did not statistically compare RTs in the presence of emotional distractors to neutral distractors, but visual inspection of data suggests that RTs for neutral distractors were similar to that of positive distractors. Experiment 2 used the same design as Experiment 1, but emotion was made taskirrelevant; rather than judging emotional expressions, participants discriminated whether a target letter was an 'S' or 'N'. Critically, in Experiment 2, they found no evidence for an emotional distraction effect - RTs did not differ in the presence of negative, neutral and positive distractors. This study also suggests that emotional distraction only emerges when distractors share emotional features of targets.

Finally, Okon-Singer and colleagues (2007) investigated emotional distraction using a spatial cueing task in which a predictive peripheral cue preceded a lateral target letter. At the same time as the target appeared, a distracting picture (negative, neutral or positive) was 
presented for $100 \mathrm{~ms}$ in the opposite location. Positive distractors were not analysed, but were included to reduce possible mood effects induced by repeated viewing of negative images. When the target appeared in the cued (i.e., attended) location and the image in the uncued (i.e., unattended) location, negative distractors had no effect on responses to the target relative to neutral distractors. However, when the target appeared in the uncued (i.e., unattended) location and the image appeared in the cued (i.e., attended) location, negative distractors slowed target responses relative to neutral distractors. This suggests that emotional distractors are only more potent than neutral distractors when presented in an attended, and thus relevant, location. In accordance with Lichtenstein-Vidne (2012), and Barratt \& Bundesen (2012) this study suggests that truly irrelevant emotional distractors are no more potent than irrelevant neutral distractors.

\section{Evidence for Task-Irrelevant Emotional Distraction.}

In contrast to the findings reported above, two studies have found behavioural evidence for task-irrelevant emotional distraction (Fox, Yates \& Ashwin, 2012; Yates, Ashwin \& Fox, 2010). Yates and colleagues (2010) examined distraction in a flanker task. In this task, participants discriminated whether a target letter was an ' $\mathrm{X}$ ' or a ' $\mathrm{Z}$ ' under low (target letter embedded in homogenous letters - 'o's) or high (target letter embedded in heterogeneous letters - ' $\mathrm{k}$ ', 's', 'm', and 'v') perceptual load conditions, while ignoring flankers presented above or below the letter display. Flankers (i.e., distractors) were angry faces previously conditioned with an aversive shock (high emotionality), non-conditioned angry faces (relatively lower emotionality), and neutral faces. An emotional distraction effect was observed; responses were slowest in the presence of conditioned angry faces, and were slower in the presence of non-conditioned angry faces than neutral faces. This effect was specific to low perceptual load conditions, suggesting that irrelevant emotional distraction only emerges when sufficient perceptual resources are available to process the distractors (see Lavie, 1995, 2005). Fox et al. (2012) replicated and extended the study by Yates et al. (2010). First, they compared groups of low and high anxious participants. They found that only low-anxious individuals showed this irrelevant emotional distraction effect. Curiously, high anxious individuals were more distracted by neutral, than emotional, distractors suggesting they adopt a strong avoidance strategy to deal with irrelevant aversive stimuli (consistent with findings linking anxiety to threat avoidance; e.g., see Koster, Crombez, Verschuere, Van Damme \& Wiersema, 2006). Second, they included happy faces (in Experiment 2) as distractors. They found that irrelevant emotional distraction was limited to negative distractors - RTs did not differ in the presence of positive (happy) and neutral 
distractors. Taken together, these studies suggest that entirely task-irrelevant negative distractors are more potent than positive or neutral distractors.

While the studies by Yates et al. (2010) and Fox et al. (2012) are the only studies that provide behavioural evidence for task-irrelevant emotional distraction, there is electrophysiological evidence that irrelevant emotional distractors are processed to a greater extent than neutral distractors (Ikeda, Sugiura \& Hasegawa, 2013). Ikeda et al. (2013) measured electroencephalography (EEG) while participants completed an emotional flanker task. In this task, participants searched for a letter at fixation (under low and high perceptual load conditions) and were told to ignore flanking faces that appeared on either side of the letter display. Flankers were either neutral-neutral face pairs, or neutral-angry face pairs. Event related potentials (ERPs) were extracted from the EEG data in order to examine neural responses to emotional and neutral distractors. There was no difference between RTs in the presence of angry and neutral distractors. However, ERP measures revealed that attention was biased toward emotional distractors (as measured by the N2pc), compared to neutral distractors, under both low and high perceptual load conditions. Further, attention was sustained to emotional distractors (as measured by the late positive potential) compared to neutral distractors under low load perceptual load conditions. Although this study provides evidence that emotional characteristics of distractors are being processed, results cannot be interpreted as a distraction effect as no behavioural distraction was observed. In fact, the behavioural data of this study are in in line with studies that find irrelevant emotional distractors are no more potent than neutral distractors.

In sum, there is limited evidence for emotional distraction effects when distractors are truly irrelevant. Only two studies have found emotional distraction effects from truly irrelevant distractors (Fox et al., 2012; Yates et al., 2010) - and one study found that this effect was specific to negative emotional distractors (Fox et al., 2012). However, clear evidence for task-irrelevant emotional (both positive and negative) distraction was recently attained in our lab (Grimshaw, Kranz, Carmel, Moody \& Devue, in prep.). To determine whether task-irrelevant emotional stimuli are more potent distractors than irrelevant neutral stimuli, a flanker paradigm was adapted from Forster and Lavie (2008a, 2008b) who used the task to show attentional capture by task-irrelevant neutral distractors. On each trial, participants discriminated whether a target letter embedded in a circle of 'o's (i.e., under low perceptual load conditions) was an X or an N. On 50\% of trials an irrelevant distractor was presented either above or below the letter display. Including distractor-absent trials enabled us to calculate a baseline measure of RT in the absence of a distractor. Previous studies of 
emotional distraction typically do not include distractor-absent trials, and measure emotional distraction by determining whether RTs are slower on emotional-distractor trials than on neutral-distractor trials. Incorporating distractor-absent trials allows for the magnitude of neutral distraction and emotional distraction to be directly compared (i.e., the change in RT between distractor-absent and present trials). The lower distractor frequency rate of $50 \%$ was chosen to increase levels of distraction. Under high distractor frequency conditions proactive control is known to be more readily engaged, which could lead to decreased distraction overall (Braver, 2012). In fact, one possible reason previous studies have not reliably found emotional distraction effects is because distractors are present on every (or a high proportion of) trial(s), potentially allowing for proactive control of both neutral and emotional distraction.

In our study (Grimshaw et al., in prep), distractors were neutral (scenes depicting people), positive (erotic scenes involving heterosexual couples) and negative (mutilated bodies) images taken from the International Affective Picture System (IAPS; Lang, Bradley \& Cuthbert, 2008). Emotionality (emotional vs. neutral) of distractors was blocked and manipulated within subjects, and the valence (positive vs. negative) of the emotional distractors was manipulated between subjects. Positive and negative stimuli were matched for arousal (based on ratings reported in Lang et al., 2008). Participants completed two sets of blocks; in one set, distractors were intact images, and in the other set distractors were pixelscrambled versions of the intact images so that the low-level perceptual features of the picture remained, but the content of the image was not discernable. Results showed that irrelevant flankers were distracting - RTs were slower on distractor-present, relative to distractor-absent trials. Importantly, emotional images (both positive and negative) were significantly more distracting than neutral images. Further, this emotional distraction effect only held when the pictures were intact; scrambled images were all equally as distracting as neutral images. Taken together, results suggest that emotional stimuli - both positive and negative - are more distracting than neutral stimuli when they are completely task-irrelevant. Further, this emotional distraction effect was due to the meaningful content of the pictures, rather than low-level perceptual features.

\section{Control of Emotional Distraction}

An established paradigm that successfully produces emotional distraction effects from entirely task-irrelevant distractors allows me to examine how we are able to control emotional distraction - are we doomed to always experience emotional distraction, or can we control it? Indeed, the absence of task-irrelevant emotional distraction in some of the 
paradigms described above suggests that emotional distraction can be successfully controlled under certain conditions. Previous research indicates that neutral distraction can be effectively controlled under conditions that evoke proactive, over reactive, control (Braver, 2012; Chao, 2011, Lee \& Chao, 2012; Munneke et al., 2008; Padmala \& Pessoa, 2011; Van der Stigchel \& Theeuwes, 2006). However, it is not known whether we can proactively control emotional distraction as effectively as neutral distraction.

It is theorised that emotional attention - the attentional benefit that emotional information receives over neutral information - is underpinned by emotion-specific processing systems (Pourtois et al.,, 2013; Vuilleumier, 2005). Emotion is argued to trigger early amygdala responses that enhance neural representation of the emotional stimulus, leading to its attentional prioritisation (commonly referred to as attentional 'gain'). The amygdala is ideally placed to be a hub for emotional processing; it is a well-connected region with wide-reaching connections across the brain (LeDoux, 2003). Evidence for the primary role of the amygdala in emotional processing (compared to non-emotional processing) comes from both neuroimaging studies (e.g., Morris et al., 1998; Sander, Grafman \& Zalla, 2003; see Phan, Wager, Taylor \& Liberzon, 2002 for a meta-analysis) and lesion studies (e.g., Adolphs, Tranel, Damasio \& Damasio, 1994,1995; Anderson \& Phelps, 2001; Vuilleumier, Richardson, Armony, Driver \& Dolan, 2004). Biased competition models argue that the amygdala biases competition for neural representation towards emotional (over neutral) information through feedback pathways to sensory processing areas (e.g., Pessoa, 2009; Vuilleumier, 2005).

Some researchers argue that amygdala-driven emotional attention is independent of top-down control (e.g., Brosch, Pourtois, Sander \& Vuilleumier, 2011; Viviani, 2013; Vuilleumier, 2005). If so, then proactive mechanisms should not be able to control irrelevant emotional distraction as effectively as irrelevant neutral distraction. Proactive control reduces distraction in a top-down manner by enhancing goal-relevant target processing and/or suppressing distractor processing (Braver, 2012). However, if emotional attention is independent of top-down control, then processing of emotional distractors should still be enhanced relative to neutral distractors, despite an overall reduction in distraction when proactive strategies are engaged (see Figure 1 for a visual depiction of this prediction).

Other researchers argue that emotional attention is not independent of top-down control systems (e.g., Pessoa, 2009; Pessoa, Kastner \& Ungerleider, 2002; Pourtois et al., 2013). If so, then proactive mechanisms might be able to control irrelevant emotional distraction as effectively as irrelevant neutral distractions. If emotional attention can be 
modulated by top-down systems, both emotional and neutral distraction would be equally low (or eliminated) when proactive strategies are engaged. Looked at another way, the attentional gain associated with emotion (i.e., an emotional distraction effect) should only emerge under conditions in which reactive control is engaged (see Figure 2 for a visual depiction of this prediction).

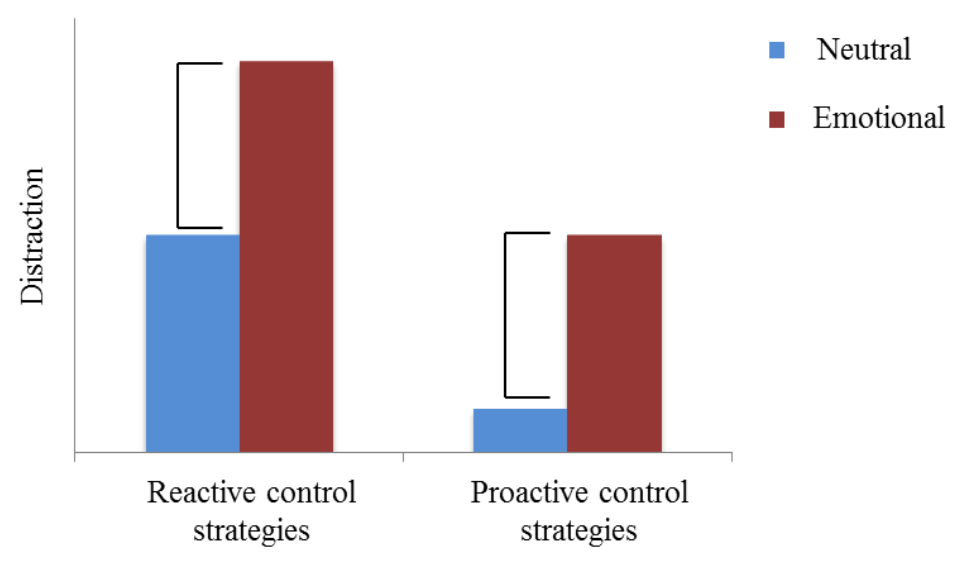

Figure 1. An example of what experimental data might look like if emotional attention is independent of top-down control. As depicted, a shift towards proactive, and away from reactive, cognitive control strategies should decrease overall levels of distraction. However, if the attentional gain that emotional information receives via the emotional attention system cannot be modulated by top-down control mechanisms, then emotional stimuli should be equally more distracting than neutral stimuli regardless of whether proactive or reactive control is engaged.

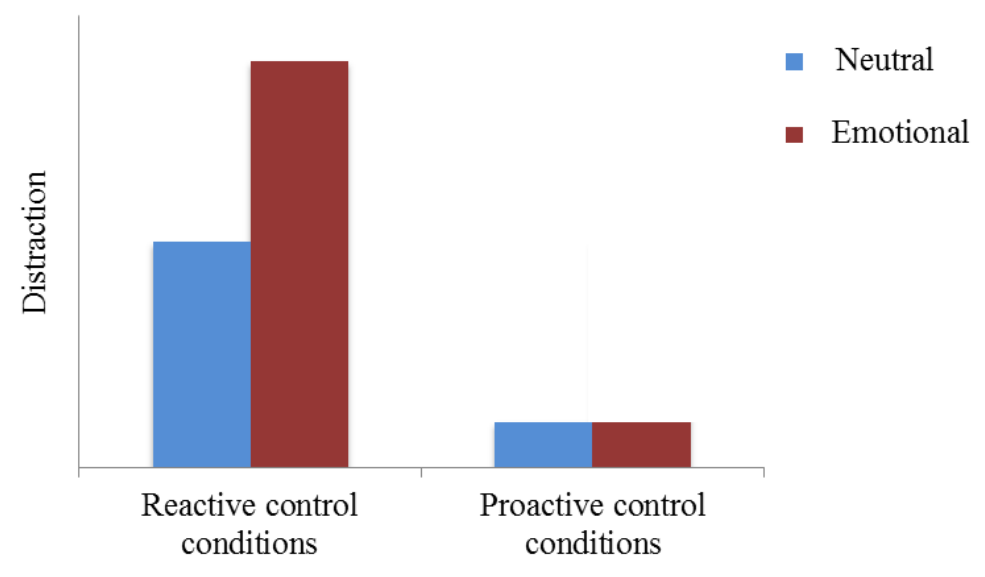

Figure 2. An example of what experimental data might look like if emotional attention can be modulated by top-down control. Critically, pronounced attentional gain by emotional stimuli (i.e., an emotional distraction effect) should be observed when reactive, but not proactive, cognitive control is engaged; when proactive mechanisms are engaged, emotional distraction should be as effectively controlled as neutral distractions because they should not receive attentional gain via the emotional attention system. 


\section{Empirical Evidence: Can Emotional Stimuli be Effectively Controlled?}

No studies have directly compared the effects of top-down control on emotional and neutral distractions in a task-irrelevant distraction paradigm - which is not surprising given the lack of research on irrelevant emotional distraction. However, there is some suggestion that emotional distractions can be proactively controlled when distractors share properties with targets, or are presented in attended locations. Blagrove and Watson $(2010,2014)$ found that previewing emotional distractors facilitated visual search time relative to a no-preview condition. This suggests that emotional distractors become less distracting when they are anticipated. Also in a visual search task, Berggren, Koster and Derakshan (2012) found that impairing participants' ability to engage in proactive control (by taxing cognitive control resources with a concurrent working memory task) increased emotional distraction. This suggests that emotional distraction was more effectively controlled under conditions in which participants were able to engage in proactive control. Similarly, Holmes, Mogg, de Fockert, Nielsen and Bradley (2014) reported increased neural processing of emotional distractors (as indexed by the N2pc and late positive potential ERP components) when cognitive control resources were taxed with a concurrent working memory task. While these studies suggest that emotional distraction can be affected by top-down control processes, none of these studies directly compared the effect of top-down control on emotional and neutral distraction. Despite demonstrating reduced emotional distraction under proactive control conditions, they did not address the question of whether emotional distractors are equally more distracting than neutral distractors under both proactive and reactive control conditions.

A handful of studies have directly compared the effect of top-down control on emotional and neutral distraction - although again not in paradigms in which distractors are entirely irrelevant. In the spatial cueing task described above, Okon-Singer et al. (2007) examined the effect of location cueing on emotional distraction. Announcing the location of an upcoming distractor allows participants to anticipate the distractor location, encouraging proactive control strategies. Indeed, location cueing has been found to reduce distraction in neutral distraction tasks (e.g., Lee \& Chao, 2012; Munneke et al, 2008). Okon-Singer and colleagues (2007) indirectly indicated where upcoming distractors (emotional or neutral) would be on most trials, by predictively indicating the location of an upcoming target. Emotional images were more distracting than neutral images when the predictive cue was invalid - i.e., when the distractor location was invalidly indicated. However, on validly cued trials, when the distractor location was correctly indicated and could thus be anticipated, there was no difference between RTs in the presence of emotional and neutral distractors (i.e., no 
emotional distraction effect). This suggests that proactive control mechanisms may control emotional distraction as effectively as neutral distraction, indicating that top-down control systems can modulate emotional attention. However, an alternative explanation of results is that emotional distraction was simply more pronounced in attended (cued) as opposed to unattended (non-cued) locations.

In four experiments, Augst and colleagues (2014) attempted to manipulate proactive and reactive control processes to examine top-down control of emotional distraction. Participants judged whether the orientation of two bars presented either side of central distractor (negative, positive and neutral (scrambled) images) was the same or different. In this task, distractors are not truly irrelevant to the participant, as they are presented at fixation (an attentionally prioritised location; Beck \& Lavie, 2005), but they are irrelevant to the task. A typical negative emotional distraction effect was observed across all experiments; target detection was slower when distractors were negative, as compared to positive and neutral. The absence of a positive emotional distraction effect is not entirely surprising as low arousal positive images were used. In each experiment they manipulated proactive control in a different manner. In Experiment 1 they examined distraction on a trial-by-trial basis to assess whether encountering a distractor on one trial engages proactive mechanisms to better control distractors of the same valence on subsequent trials. In experiments 2 and 3 they cued the valence of the upcoming distractor. Anticipating the valence of a distractor may allow one to proactively control it. Indeed, cueing the identity of distractors has been found to reduce distraction (Blagrove \& Watson, 2010, 2014), suggesting that it engages proactive control mechanisms. Valence-cues (e.g., the word positive) were compared to non-word cues (e.g., puzkuri). Finally, in experiment 4, they manipulated distractor proportions; in a given block, $80 \%$ of trials consisted of one distractor valence (e.g., negative), and $20 \%$ of the other valences (e.g., positive and neutral). Increasing the frequency of a distractor type (e.g., negative distractors) should evoke proactive control as it becomes more beneficial to exert resources to control attention to frequent distractors (Braver, 2012).

Across experiments Augst et al. (2014) found that negative emotional distractors were more potent than neutral and positive distractors, even in the proactive control conditions. However, this cannot be interpreted as evidence that emotional attention is independent of top-down control because the results also suggest that they did not successfully engage proactive control with their manipulations. In Experiment 1, emotional distractors increased negative distraction (RT) on the following trial. This suggests that, rather than engaging proactive control, a sensitisation effect occurred whereby encountering an emotional stimulus 
increased sensitivity to emotional distraction (see Kunde \& Mauer, 2008 for a similar effect). In experiments 2 and 3 overall distraction (as measured by RTs) was not affected by announcing the valence cue of the upcoming distractor. If proactive control was effectively evoked, then overall levels of distraction should have decreased. Indeed, previous research suggests that announcing the valence of a distractor does not effectively evoke proactive control - sometimes it even has a paradoxical effect whereby it increases distraction (Kleinsorge, 2007, 2009), possibly by priming participants to monitor for emotion. Finally, in experiment 4, they found that presenting a high proportion of negative (as compared to positive or neutral) distractors reduced the difference between RTs in the presence of negative and neutral/positive distractors. At face value, this suggests that under proactive control conditions (i.e., when there was a high probability of the distractor being negative) emotional distraction was controlled as effectively as neutral distraction. However, visual inspection of RTs shows that this interaction was not due to reduced negative distraction, but rather increased RTs on neutral and positive trials embedded within the "high negative" block, leading to equal slowing for all distractor types. Taken together, the results of the experiments conducted by Augst et al. (2014) are far from conclusive. As their manipulations of proactive control were not successful, results do not indicate whether emotional distraction (even if the distractors are not truly irrelevant) can be modulated by top-down control systems.

Padmala and Pessoa (2014) also investigated whether proactive mechanisms can control emotional distraction as effectively as neutral distraction. They used a distraction task similar to that used by Augst et al. (2014). Participants judged whether the orientation of two bars presented either side of a central distractor was the same or different. Distractors were negative (bodily mutilations) or neutral images. Proactive control was evoked on some trials by rewarding good performance on the target orientation task. Reward incentive is thought to encourage the use of proactive control (Locke \& Braver, 2008; Pessoa \& Engelmann, 2010), and has been found to reduce distraction from irrelevant neutral stimuli (e.g., Padmala \& Pessoa, 2011; Van den Berg, Krebs, Lorist \& Woldorff, 2014). Each trial began with a cue that indicated whether a monetary reward was available for that trial. On reward trials participants could earn 20 cents for fast and accurate responses. A typical emotional distraction effect was observed in the no-reward condition; responses on the bar orientation task were slower in the presence of negative than neutral distractors. Critically, this emotional distraction effect was abolished in the reward condition; RT did not differ in the presence of emotional and neutral images. This suggests that negative distraction can be proactively 
controlled as effectively as neutral distraction. Indeed, this study provides perhaps the most compelling evidence so far that emotional attention is not independent of top-down control. However, Padmala \& Pessoa (2014) did not include a distractor-absent baseline condition so could not directly compare changes in emotional distraction to changes in neutral distraction. Further, because positive distractors were not used, this study does not tell us whether proactive mechanisms can effectively control positive distraction. Finally, it should be noted that this study did not examine truly irrelevant distraction; distractors were presented in an attended location - fixation (see Beck \& Lavie, 2005). The study did not address the question of whether proactive mechanisms can control irrelevant emotional distraction as effectively as neutral distraction.

\section{The Current Experiments}

To summarise, no studies have assessed whether irrelevant emotional distraction can be controlled as effectively as neutral distraction. It is not known whether emotional attention to irrelevant distractors is independent of top-down proactive control, or whether emotional distraction can be modulated by proactive mechanisms as neutral distraction can - although recent evidence from a paradigm in which distractors were presented in an attended location supports the latter (Padmala \& Pessoa, 2014). Furthermore, because most research has focused on negative (threat-related) distractors, it is not clear whether positive information of equivalent arousal (which produce similar distraction effects; Grimshaw et al., in prep) can be controlled through similar mechanisms. To address these gaps in the literature, I present two experiments that examined how irrelevant emotional distraction can be controlled.

Both experiments used the same basic emotional distraction paradigm (adapted from Forster \& Lavie, 2008a, 2008b) that has previously been used in our lab to show that emotional distractors are more potent than neutral distractors when present on half of trials. Under low perceptual load conditions, participants distinguished whether an $\mathrm{X}$ or an $\mathrm{N}$ was present amongst a circle of 'o's presented briefly at fixation (100ms). Distractors were randomly presented at the same time, either above or below the letter display - locations that were never relevant to the task. Distractors were negative (bodily mutilations), positive (erotic images involving heterosexual couples) and neutral (scenes including people) images taken from the IAPS (Lang et al., 2008). Negative and positive images were high arousal, and matched for arousal based on normative ratings reported in Lang et al. (2008). This paradigm has several advantages over others that have been used to study emotional distraction: 1) the distractors are completely task-irrelevant; 2) a neutral distraction condition is included, enabling comparison of emotional and neutral distraction; 3) distractor-absent trials are 
included, which enables calculation of distraction from both neutral and emotional distractors (distractor-present compared to distractor-absent trials); and 4) positive distractors have similar subjective arousal ratings to negative distractors.

Distractor frequency was manipulated to evoke reactive and proactive control (see Augst et al., 2014; Braver, 2012; Burgess \& Braver, 2010; Ridderinkhof, 2002). Increasing distractor frequency is argued to encourage proactive control strategies, leading to decreased distraction (Braver, 2012; Burgess \& Braver, 2010). Indeed, one previous study has shown that decreasing distractor frequency increases distraction using a neutral version of the current paradigm (Forster \& Lavie, 2008a). In my experiments, distractors were presented on either a low proportion (25\%) or a high proportion (75\%) of trials to evoke reactive and proactive cognitive control strategies, respectively. Based on our previous research (Grimshaw et al. in prep), I expected more distraction from emotional than neutral distractors in the reactive control condition. The critical issue was how the shift to proactive control affected emotional distraction. If emotional attention and top-down control are independent processes, then I would expect distractor probability and emotionality to be additive, with greater emotional than neutral distraction in both high and low distractor probability conditions. However, if emotional attention can be modulated by top-down processes (i.e., proactive processes can control both neutral and emotional distraction), then I expect distractor probability and emotionality to interact to produce equivalently low levels of distraction from emotional and neutral distractors under low distractor probability conditions.

Experiment 1 was a behavioural experiment that tested whether proactive mechanisms can control irrelevant emotional distractors as effectively as neutral distractors. Experiment 2 was an ERP study that extended Experiment 1 to examine the neural correlates of emotional processing under reactive and proactive conditions. EEG was measured while participants performed the behavioural distraction task. Two emotion-sensitive ERP components were examined: 1) the early posterior negativity (EPN), which is thought to index early recruitment of attentional resources for perceptual processing of a stimulus, and 2) the late positive potential (LPP), which is thought to reflect sustained attention to stimuli (Hajcak, Weinberg, MacNamara \& Foti, 2012). Both the EPN and the LPP are potentiated for emotional, compared to neutral, stimuli (Hajcak et al., 2012). If proactive mechanisms control emotional distraction by preventing early perceptual processing of emotional distractors, then evoking proactive control (i.e., increasing distractor frequency) should attenuate emotional modulation of both the EPN and the LPP. Alternatively, if proactive control does not affect early perceptual processing of emotional distractors, but rather 
prevents sustained attention to emotional distractors, then emotional modulation of the LPP should be attenuated by the use of proactive control strategies (i.e., under high distractor frequency conditions) but emotional modulation of the EPN should be unaffected. An additional component - the anterior $\mathrm{N} 2$-, which is thought to index cognitive control processes (Nieuwenhuis, Yeung, van den Wildenberg \& Ridderinkhof, 2003), was also examined in an exploratory manner to see if it reliably distinguished engagement of proactive compared to reactive control processes.

Across studies, only women were recruited to keep the sample as homogenous as possible; significant sex differences in emotional processing have been reported (Bradley, Codispoti, Sabatinelli \& Lang, 2001; McRae, Ochsner, Mauss, Gabrieli \& Gross, 2008; Syrjänen \& Wiens, 2013). Further, as depression and anxiety are characterised by alterations in emotional processing and attentional control (MacLeod, Mathews, \& Tata, 1986; Mogg \& Bradley, 2005; Mogg, Bradley \& Williams, 1995), only participants with no current or previous history of depression or anxiety were included.

\section{Experiment 1}

The aim of Experiment 1 was to determine whether proactive control mechanisms can control irrelevant emotional - both positive and negative - distraction as effectively as neutral distraction. The few studies that have examined control over emotional distraction have not adequately addressed this question, either because they did not compare emotional distraction to neutral distraction (e.g., Blagrove \& Watson 2010, 2014; Berggren et al., 2012; Holmes et al., 2014), did not clearly manipulate cognitive control (e.g., Augst et al., 2014), or used a paradigm in which distractors were task-relevant or in attended locations (Okon-singer et al., 2007; Padmala \& Pessoa, 2014). It is not clear whether irrelevant emotional distraction is independent of, or can be modulated by, top-down proactive control systems. To that end, in Experiment 1 a task-irrelevant distraction paradigm was used to determine whether proactive mechanisms can effectively control attention to irrelevant emotional distractors. Experiment 1 further compared control of both positive and negative distraction.

Participants completed an emotional distraction task adapted from Forster and Lavie (2008a, 2008b). On each trial participants determined whether a target letter was an X or an $\mathrm{N}$ among an array of o's, while ignoring peripheral distractors. Participants were randomly assigned to two conditions; low distractor frequency (distractors presented on $25 \%$ of trials), or high distractor frequency (distractors presented on $75 \%$ of trials) - designed to evoke reactive and proactive cognitive control strategies, respectively. Distractors included negative 
(bodily mutilations), positive (erotic scenes involving heterosexual couples) and neutral (scenes with people) images taken from the IAPS (Lang et al., 2008).

Two predictions were made. First, if the distractor frequency manipulation effectively evokes proactive and reactive control, then more distraction (i.e., more slowing on distractorpresent compared to distractor-absent trials) should be observed under low distractor frequency conditions (i.e., when reactive control dominates) than under high distractor frequency conditions (i.e., when proactive control is engaged; Braver, 2012). Second, based on previous findings from emotional distraction paradigms (Fox et al., 2012; Grimshaw et al., in prep; Y Yates et al., 2010), I predicted that emotional stimuli (both positive and negative) would cause more distraction than neutral stimuli, at least in the reactive condition. Importantly, if emotional attention is independent of top-down control (Brosch et al., 2011; Viviani, 2013; Vuilleumier, 2005), then cognitive control and emotion should produce additive effects on distraction - that is, emotional distractors should be equally more distracting than neutral distractors under both high and low distractor frequency conditions. The logic of this prediction stems from the hypothesis that emotion biases attentional selection of the image, making it a potent distractor (Pessoa, 2009; Pourtois et al., 2013) Proactive control can reduce that distraction through enhancing goal-relevant target processing and/or suppressing distractor processing (Braver, 2012). However, if emotional attention is independent of top-down control, then the attentional gain emotional distractors receive over neutral distractors (i.e., emotional distraction) should remain constant, despite an overall reduction in distraction under proactive control conditions. Alternatively, if emotional attention can be modulated by top-down control (Pessoa, 2009; Pessoa et al., 2002; Pourtois et al., 2013) - i.e., emotional distractors can be as effectively controlled proactively as neutral distractors -, then both emotional and neutral distraction should be equally low under high distractor frequency conditions.

To ensure that participants across conditions were equated on symptoms of depression and anxiety (which is known to affect distraction; MacLeod et al., 1986; Mogg \& Bradley, 2005; Mogg et al., 1995), the Mini Mood and Anxiety Symptoms Questionnaire (MiniMASQ; Clarke \& Watson, 1995) was administered. Further, to ensure that attentional control ability was equated across conditions, participants completed the Attentional Control Scale (Derryberry \& Reed, 2002). 


\section{Method}

\section{Participants}

Sixty-four women (9 left-handed) ranging from 18 to 23 years of age $(M=18.66, S D$ $=1.01$ ) with normal or corrected-to-normal vision were recruited from the undergraduate psychology pool at Victoria University of Wellington. Participants reported not receiving treatment (past or current) for depression or anxiety. Participants were randomly assigned to the high distractor frequency or low distractor frequency condition. They received course credit for their participation, and provided written informed consent prior to participation. This study was approved by the Human Ethics Committee of the School of Psychology, Victoria University of Wellington (Wellington, New Zealand).

\section{Materials}

\section{Distraction Task.}

Task Procedure. The distraction task was adapted from Forster and Lavie (2008a, 2008b). Participants discriminated whether a capitalised target letter was an ' $\mathrm{X}$ ' or an 'N'. The target letter (font: Arial; font size: 24 ; subtending $0.67^{\circ}$ of visual angle) was randomly presented in one of six possible positions arranged in a circle around fixation, with a radius of $1.75^{\circ}$ eccentricity. Lowercase 'o's (font: Arial; font size: 8 ; subtending $0.22^{\circ}$ of visual angle) appeared in the five non-target positions on each trial. See Figure 3 for a visual depiction of the trial procedure. Each trial began with a central fixation cross of a random duration between 416 and $834 \mathrm{~ms}$. Following fixation, the visual letter display was presented for $100 \mathrm{~ms}$. Subjects were required to press 1 if the target was an ' $\mathrm{X}$ ' and 2 if the target was an ' $N$ ' with the index and middle finger of their right hand, respectively. On a certain proportion of trials a distractor image (positive, neutral or negative) was simultaneously presented above or below the visual letter display, such that the nearest edge of the picture was $3.34^{\circ}$ above or below fixation. Combinations of the target location, target letter $(\mathrm{X}$ or $\mathrm{N})$ and distractor location were randomized. A 2000ms fixed response window (from onset of the letter display) was used, after which feedback (100ms duration) was displayed: 'correct' written in green or 'incorrect' written in red (font: Arial, font size: 18). A jittered inter-trial-interval was used, ranging between $416 \mathrm{~ms}$ and $834 \mathrm{~ms}$. 


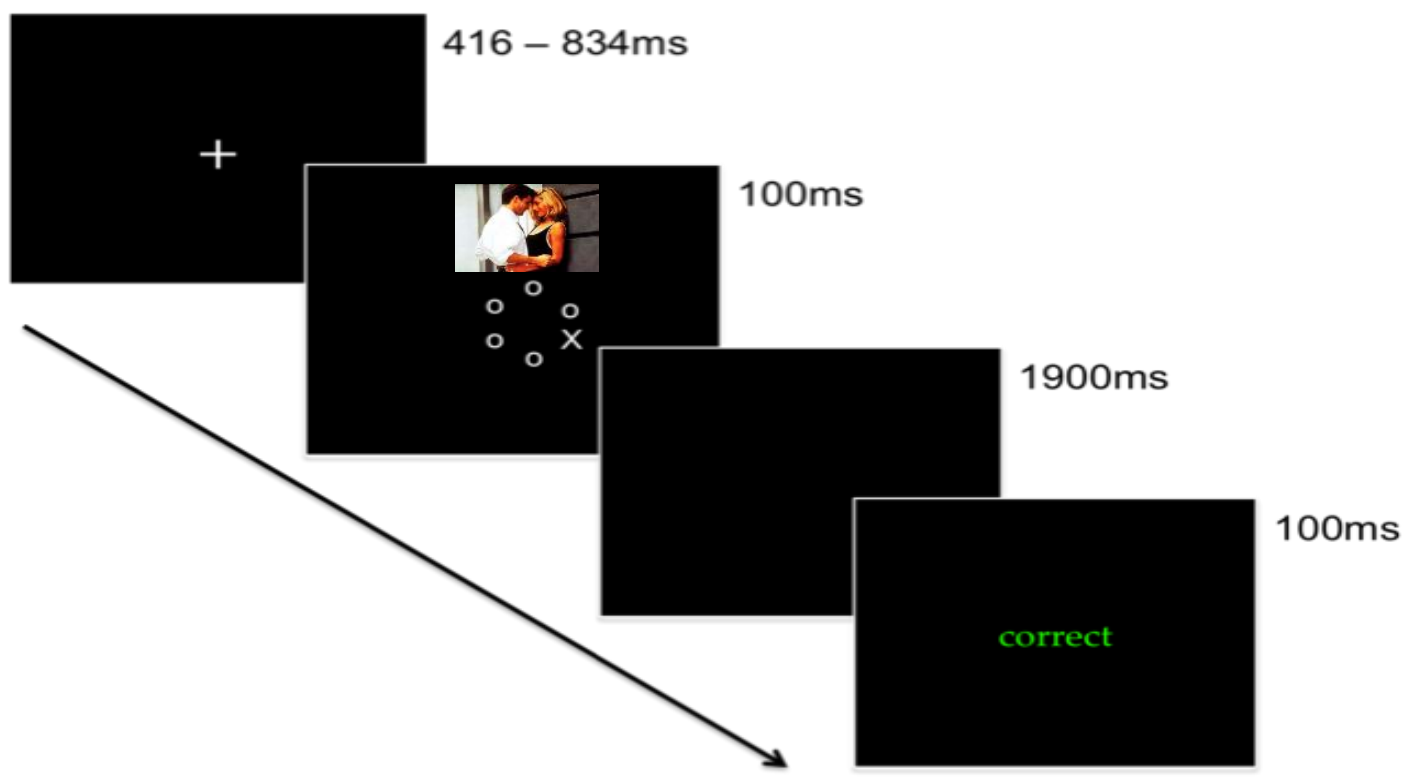

Figure 3. An example of a typical trial procedure in the distraction task.

Note. Figure is for demonstrative purposes only; stimuli are not depicted in actual size or ratio used.

See Figure 4 for a schematic representation of the blocking procedure. Distractor frequency was manipulated between subjects ( $25 \%$ or $75 \%$ of trials). The distraction task was completed in two halves; four blocks of trials (48 trials per block) were completed in each half, with distractor emotionality (emotional or neutral) blocked in an ABBA fashion (see Figure 4). Block order was the same for a given participant in each half of the experiment. In each half, emotional distractors were either positive or negative, with valence order counterbalanced across subjects. Between the first and second half of the experiment, participants completed a filler task (a Sudoku puzzle) to reduce any carryover effects of picture valence from the previous blocks. Subjects were explicitly informed how often distractors would be presented before beginning the experiment ${ }^{1}$. No distractor image was repeated before all other images of that category had been presented. Before beginning the distraction task, participants completed 12 distractor-absent practice trials to familiarise themselves with the letter identification task. Participants were told to respond as quickly as possible without making mistakes.

\footnotetext{
${ }^{1}$ Participants were informed how often distractors appeared to encourage the use of either proactive or reactive control strategies from the beginning of the experiment. Critically, I wanted them to be aware of how often distractors would appear.
} 


\begin{tabular}{|c|c|c|c|c|c|c|c|c|c|}
\hline \multirow[b]{2}{*}{ Blocking } & \multicolumn{4}{|c|}{$\begin{aligned} \text { Valence }= & \text { Negative or Positive } \\
& (192 \text { trials })\end{aligned}$} & \multirow{4}{*}{ 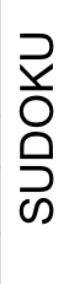 } & \multicolumn{4}{|c|}{$\begin{aligned} \text { Valence }= & \text { Positive or Negative } \\
& (192 \text { trials })\end{aligned}$} \\
\hline & Block 1 & Block 2 & Block 3 & Block 4 & & Block 5 & Block 6 & Block 7 & Block 8 \\
\hline Order 1 & Emotional & Neutral & Neutral & Emotional & & Emotional & Neutral & Neutral & Emotional \\
\hline Order 2 & Neutral & Emotional & Emotional & Neutral & & Neutral & Emotional & Emotional & Neutral \\
\hline
\end{tabular}

Figure 4. Schematic representation of the blocking procedure and counterbalances for the distraction task. Within each block, participants saw a mixture of distractor-present and distractor-absent trials, as determined by their distractor probability condition.

Picture Stimuli. Picture stimuli for the distraction task were 36 colour images taken from the IAPS (Lang et al., 2008). Stimuli were 12 negative pictures (mutilations), 12 positive pictures (erotic images involving heterosexual couples) and 12 neutral pictures (scenes depicting people). See Appendix A for a complete list of the images, as well as individual normative valence and arousal ratings collected from female raters for each picture (from Lang et al., 2008). See Table 1 for the mean valence and arousal ratings for each image set. A one-way ANOVA was conducted on valence ratings for the images sets, which showed a significant difference between mean valence ratings for positive, neutral and negative images, $F(2,33)=475.62, p<.001$. Follow-up tests indicated that positive images were rated as more positive than neutral images, $t(22)=6.82, p<.001 d=2.75^{2}$, which were more positive than negative images, $t(22)=29.27, p<.001, d=11.91$. A second one-way ANOVA showed significant differences in arousal ratings, $F(2,33)=372.64, \mathrm{p}<.001$. Follow-up tests showed no significant difference between positive and negative images, $t(22)=1.53, p=.14$. However, neutral images were rated lower in arousal than both negative images, $t(22)=$ 22.70, $p<.001, d=9.27$, and positive images, $t(22)=25.91, p<.001, d=10.61$. Pictures were matched for luminance and contrast using Matlab SHINE toolbox (Willenbockel et al., 2010), and were chosen such that they had the same dimensions. Pictures subtended $6.68^{\circ} \mathrm{x}$ $6.68^{\circ}$ of visual angle and were presented in color on a black background.

\footnotetext{
${ }^{2}$ For all t-tests in this thesis, Cohen's d is calculated using the difference between the means, divided by the pooled standard deviation (Dunlap, Cortina, Vaslow, \& Burke, 1996).
} 
Table 1

Mean (SD) valence and arousal ratings for the picture stimuli used in the distraction task.

\begin{tabular}{lll}
\hline & Valence & Arousal \\
Picture set & M (SD) & M (SD) \\
\hline Positive & $6.23(.52)$ & $6.31(.29)$ \\
Negative & $1.64(.21)$ & $6.53(.42)$ \\
Neutral & $5.01(.34)$ & $3.07(.32)$ \\
\hline
\end{tabular}

Note. Valence and arousal scores range from 1 - 9, with 1 representing low arousal and negative valence, and 9 representing high arousal and positive valence.

\section{Questionnaires.}

The Mini Mood and Anxiety Symptoms Questionnaire. The Mini Mood and Anxiety Symptoms Questionnaire (Mini-MASQ; Clark \& Watson, 1995) was used to measure depression and anxiety symptoms. The Mini-MASQ is a 26-item questionnaire adapted from the 90-item MASQ (Clark \& Watson, 1995). The Mini-MASQ has three subscales: (1) general distress -8 items, (2) anhedonic depression - 8 items; and (3) anxious arousal - 10 items. For each item, participants rated the extent to which they had felt that way during the past week on a 5-point Likert scale $(1=$ not at all, $2=$ a little bit, $3=$ moderately, $4=$ quite a bit, 5 = extremely). Participants were also given the option to skip questions that they were not comfortable answering ${ }^{3}$. Typically, possible scores on the Mini-MASQ range from 26 to 130, and possible scores on each of the subscales are: general distress: 8 to 40; anhedonic depression: 8 to 40; and anxious arousal: 10 to 50. However, as participants were given the option to skip items on the questionnaire, individual questionnaire scores were calculated in the current study using the mean score across responses (recorded numerically), rather than calculating the sum of responses; possible scores therefore ranged from 1 to 5 for the total Mini-MASQ and each of its subscales. See Appendix B for the full list of questionnaire items.

The Attentional Control Scale. The Attentional Control Scale (Derryberry \& Reed, 2002 ) is a 20 -item questionnaire that measures general capacity for attentional control. Participants rate how much each statement applies to them on a 4-point Likert scale $(1=$ almost never, 2 = sometimes, 3 = often, 4 = always). Participants were given the option to skip questions that they were not comfortable answering. Possible scores typically range from

\footnotetext{
${ }^{3}$ While it is not normal procedure to give participants the option to skip questions, I was ethically required to provide this option.
} 
1 to 80 . However, similar to the Mini-MASQ, as participants were given the option to skip items, scores were calculated in the current study using the mean score across responses, rather than calculating the sum of responses; possible scores therefore ranged from 1 to 4 . See Appendix C for the full list of questionnaire items.

\section{Procedure and Design}

All experiments were programmed and run in E-Prime 2.0 (Schneider, Eschman, \& Zuccolotto, 2002a, 2002b). The experiment took approximately 30 minutes to complete. The distraction task was completed first, followed by the Mini-MASQ and then the Attentional Control Scale. Data were collected in groups of up to four participants per session. Dividers were used to create independent cubicles for each participant. Additionally, the entrance to each cubicle contained a curtain that was drawn during the questionnaires to create privacy. Participants completed the experiment on Dell Precision T1700 desktop computers with 24" inch AOC monitors with a resolution of 1920 by 1080 pixels, and a vertical refresh rate of $120 \mathrm{~Hz}$. Chin rests were used throughout the experiment to maintain an approximate viewing distance of $57 \mathrm{~cm}$.

The independent variables were distractor frequency (high, low; between subjects), distractor emotionality (emotional, neutral; within subjects), distractor valence (negative, positive; within subjects), and distractor presence (present, absent; within subjects). The primary dependent variable of interest was response time (RT) on the letter search task, as distraction is typically measured in RTs. However, accuracy was also included as a dependent variable - although overall levels of accuracy were expected to be very high due to the ease of the task.

\section{Results and Discussion}

\section{Questionnaires}

Question skipping rates were very low for both the Mini-MASQ (.00063\% of questions) and the Attentional Control Scale (.00078\% of questions). Questionnaire scores were used to ensure that the two groups (high and low distractor frequency) did not differ in attentional control, anxiety or depression, as these could be expected to affect emotional distraction. See Table 2 for mean questionnaire scores by distractor frequency condition. Scores on the Attentional Control Scale did not significantly differ between the low and high distractor frequency conditions, $t(62)=.49, p=.625$. Total Mini-MASQ scores also did not significantly differ between the low and high distractor frequency conditions, $t(62)=1.20, p$ $=.236$. Further, scores between conditions did not significantly differ on the three subscales 
of the Mini-MASQ: general distress, $t(62)=1.18, p=.243$; anxious arousal, $t(62)=.92, p=$ .362 ; and anhedonic depression, $t(62)=.63, p=.533$.

Table 2

Mean (SD) questionnaire scores by distractor frequency condition in Experiment 1.

\begin{tabular}{lcc}
\hline & $\begin{array}{c}\text { Low Distractor } \\
\text { Frequency }\end{array}$ & $\begin{array}{c}\text { High Distractor } \\
\text { Frequency } \\
\text { M (SD) }\end{array}$ \\
\hline Attentional Control Scale & $2.50(.38)$ & $2.55(.40)$ \\
Mini MASQ Total & $2.32(.54)$ & $2.16(.53)$ \\
Mini-MASQ: general distress & $2.20(.73)$ & $1.99(.68)$ \\
Mini-MASQ: anxious arousal & $1.74(.66)$ & $1.60(.54)$ \\
Mini-MASQ: anhedonic depression & $3.09(.88)$ & $2.95(.82)$ \\
\hline
\end{tabular}

Note. Scores range from 1 to 4 for the Attentional Control Scale, and from 1 to 5 for the Mini-MASQ, as well as the three Mini-MASQ subscales: general distress, anxious arousal, anhedonic depression.

\section{Distraction Task}

Mean RTs and overall accuracy rates were calculated for each condition. For RT measures, only correct responses longer than $200 \mathrm{~ms}$ were analysed to ensure that anticipatory responses were not included in analyses. No upper bound criterion was used for RT inclusion, as the response window was limited to $2000 \mathrm{~ms}$. The exclusion criteria led to the average removal of $3.54 \%(S D=2.28)$ of trials per participant. All participants maintained an overall accuracy rate above $85 \%$.

\section{Response Times.}

RTs (ms) were entered into a 2 (distractor frequency: low, high) x 2 (valence: negative, positive) x 2 (emotionality: emotional, neutral) x 2 (distractor presence: present, absent) mixed ANOVA. For means and standard deviations by distractor frequency, valence, emotionality, and distractor presence see Table 3. Main effects of emotionality, $F(1,62)=$ 4.83, $p=.032, \eta_{p}{ }^{2}=.07$, and of distractor presence, $F(1,62)=30.57, p<.001, \eta_{p}{ }^{2}=.33$, were observed. Additionally, there was a two-way emotionality x distractor frequency interaction, $F(1,62)=6.79, p=.011, \eta_{p}{ }^{2}=.10$, and a two-way distractor presence $\mathrm{x}$ distractor frequency interaction, $F(1,62)=17.70, p<.001, \eta_{p}^{2}=.22$. Importantly, all effects were qualified by the predicted three-way emotionality $\mathrm{x}$ distractor presence $\mathrm{x}$ distractor 
frequency interaction, $F(1,62)=5.52, p=.022, \eta_{p}^{2}=.08$. As no main effects or interactions involving valence were observed, data were collapsed across valence for the remaining analyses.

Table 3

Mean (SD) RTs (ms) for the distraction task by distractor frequency, valence, emotionality, and distractor presence in Experiment 1.

\begin{tabular}{lcccccc}
\hline & \multicolumn{3}{c}{ Low Distractor Frequency } & \multicolumn{3}{c}{ High Distractor Frequency } \\
& Distractor & Distractor & Distraction & Distractor & Distractor & Distraction \\
& Present & Absent & Index & Present & Absent & Index \\
& M (SD) & M (SD) & M (SD) & M (SD) & M (SD) & M (SD) \\
\cline { 2 - 7 } Positive & & & & & \\
Emotional & $645(108)$ & $582(60)$ & $63(78)$ & $594(91)$ & $582(78)$ & $12(40)$ \\
Neutral & $608(79)$ & $590(75)$ & $18(34)$ & $589(84)$ & $586(69)$ & $3(38)$ \\
Negative & & & & & & \\
Emotional & $658(117)$ & $591(63)$ & $67(79)$ & $588(90)$ & $576(89)$ & $12(29)$ \\
Neutral & $614(83)$ & $586(64)$ & $28(50)$ & $584(79)$ & $588(90)$ & $-4(36)$ \\
\hline Note. Distraction Index = RT on distractor-present trials - RT on distractor-absent trials
\end{tabular}

To further explore the three-way interaction, a 2 (emotionality: emotional, neutral) x 2 (distractor presence: present, absent) repeated measures ANOVA was conducted separately for the low and high distractor frequency conditions. In the low distractor frequency condition, a main effect of distractor presence was observed, $F(1,31)=28.64, p<.001, \eta_{p}{ }^{2}=$ .48. Consistent with a typical distraction effect, responses were slower on distractor-present, than distractor-absent trials. There was also a main effect of emotionality, $F(1,31)=7.96, p$ $=.008, \eta_{p}{ }^{2}=.20$, that was qualified by a significant emotionality $\mathrm{x}$ distractor presence interaction, $F(1,31)=13.85, p<.001, \eta_{p}{ }^{2}=.31$. As shown in Figure 5, there was more distraction (that is, greater slowing on distractor-present compared to distractor-absent trials) from emotional images (64ms of distraction), $t(31)=5.16 p<.001, d=0.75$, than from neutral images (22ms of distraction), $t(31)=3.58, p<.001, d=0.32$. These results replicate the emotional distraction effects reported previously using this task (Grimshaw et al., in prep) and are indicative of a typical emotional distraction effect under reactive control conditions. 
In the high distractor frequency condition, no main effects were observed. However, there was a marginal emotionality $\mathrm{x}$ distractor presence interaction, $F(1,31)=4.00, p=.056$, $\eta_{p}{ }^{2}=.11$. As shown in Figure 5, significant distraction was observed from emotional images (12ms of distraction), $t(31)=2.50, p=.018, d=0.14$, but not from neutral images ( $0 \mathrm{~ms}$ of distraction), $t(31)=.02, p=.985$. However, it should be noted that in both emotional and neutral conditions distraction was markedly reduced relative to the low distractor frequency condition, suggesting that proactive control was successfully evoked. It should also be noted that emotional distraction was only marginally greater than neutral distraction in the high distractor frequency condition. The 3-way interaction is therefore driven by the fact that the difference between emotional and neutral distraction was attenuated under proactive control conditions. Emotional distraction was controlled almost as effectively as neutral distraction, suggesting that proactive control can indeed modulate emotional attention.

In sum, these results provide support for an interaction between top-down proactive control systems and emotional attention. Emotional distractors were more potent than neutral distractors when reactive mechanisms were engaged, but when proactive mechanisms were engaged emotional distraction was controlled almost as effectively as neutral distraction.

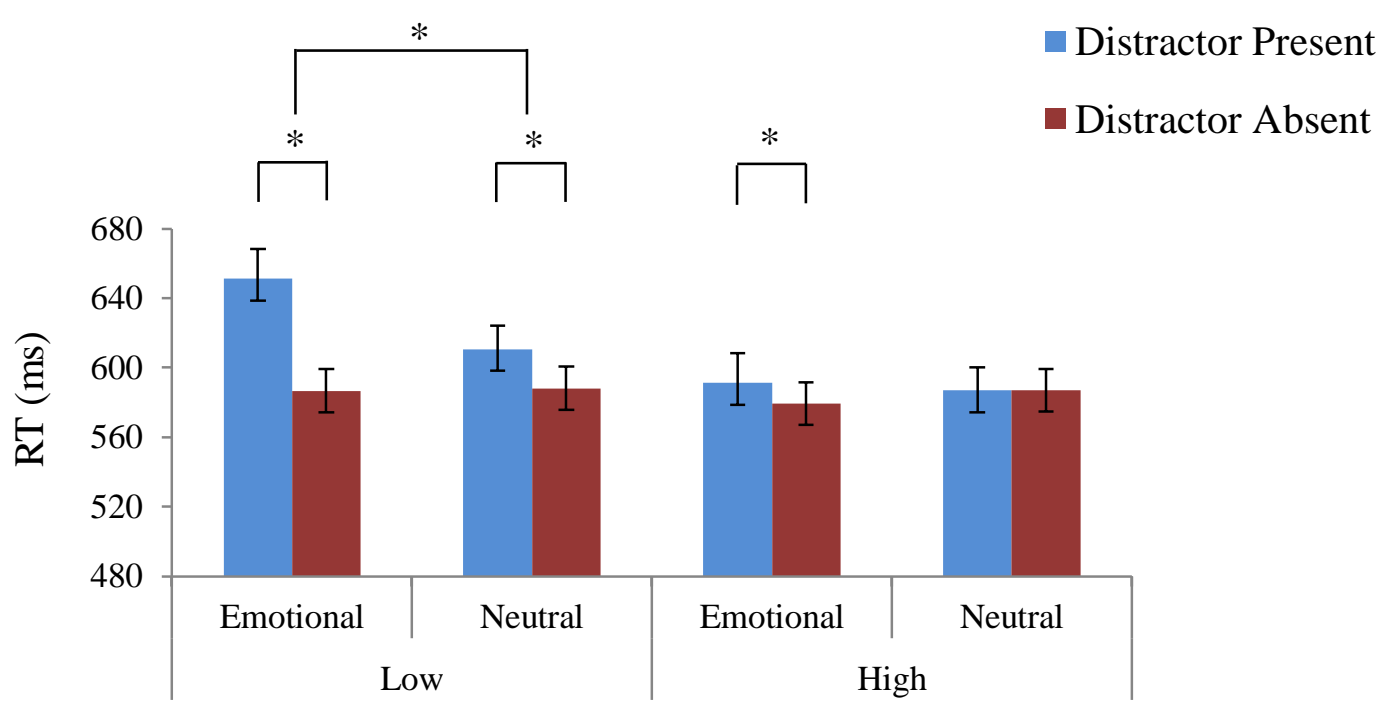

Distractor Frequency

Figure 5. Mean RTs on the distraction task by distractor frequency, emotionality and distractor presence in Experiment 1. Pronounced emotional (compared to neutral) distraction was observed in the low distractor frequency condition. In the high distractor frequency condition emotional distraction was markedly reduced.

Note. Bars represent standard errors of the mean

Note. ${ }^{*}=p<.05$ 


\section{Accuracy.}

Accuracy scores were overall very high (see Figure 6). Accuracy scores (\%) were entered into a 2 (distractor frequency: low, high) x 2 (valence: negative, positive) x 2 (emotionality: emotional, neutral) x 2 (distractor presence: present, absent) mixed ANOVA. For mean accuracy scores and standard deviations by distractor frequency, valence, emotionality, and distractor presence see Table 4 . There was a marginal main effect of valence, $F(1,62)=3.88, p=.053, \eta_{p}{ }^{2}=.06$, and significant main effects of emotionality, $F(1,62)=5.28, p=.025, \eta_{p}^{2}=.08$, and distractor presence, $F(1,62)=4.84, p=.031, \eta_{p}^{2}=$ .07 . There was also a significant emotionality $\mathrm{x}$ distractor presence $\mathrm{x}$ distractor frequency interaction, $F(1,62)=12.67, p=.001, \eta_{p}^{2}=.17$, and a valence $\mathrm{x}$ distractor presence $\mathrm{x}$ distractor frequency interaction, $F(1,62)=13.31, p=.001, \eta_{p}^{2}=.18$. All main effects and interactions were qualified by a marginal four way valence $\mathrm{x}$ emotionality $\mathrm{x}$ distractor presence $\mathrm{x}$ distractor frequency interaction, $F(1,62)=3.84, p=.055, \eta_{p}{ }^{2}=.06$ (see Figure 6).

Table 4.

Mean (SD) accuracy (\%) for the distraction task by distractor frequency, valence, emotionality, and distractor presence in Experiment 1.

\begin{tabular}{lcccc}
\hline & \multicolumn{2}{c}{ Low Distractor Frequency } & \multicolumn{2}{c}{ High Distractor Frequency } \\
& Distractor & Distractor & Distractor & Distractor \\
& Present & Absent & Present & Absent \\
& M (SD) & M (SD) & M (SD) & M (SD) \\
\hline Positive & & & & \\
Emotional & $95.93(5.17)$ & $96.60(2.75)$ & $97.20(2.98)$ & $97.64(3.36)$ \\
Neutral & $97.39(3.31)$ & $96.77(3.10)$ & $96.69(3.32)$ & $98.18(3.16)$ \\
Negative & & & & \\
Emotional & $92.94(7.68)$ & $97.38(2.36)$ & $97.91(2.68)$ & $96.22(5.00)$ \\
Neutral & $96.72(3.66)$ & $96.68(3.11)$ & $96.56(2.67)$ & $97.27(3.61)$ \\
\hline
\end{tabular}

To further examine this four way interaction, data were split by valence (positive, negative). A 2 (distractor frequency: low, high) x 2 (emotionality: emotional, neutral) x 2 (distractor presence: present, absent) mixed ANOVA was conducted separately for the positive and negative valence conditions. In the positive condition, no significant effects for accuracy were observed. In the negative condition, there was a significant main effect of distractor presence, $F(1,62)=4.74, p=.03, \eta_{p}^{2}=.07$, a significant distractor presence $\mathrm{x}$ 
distractor frequency interaction, $F(1,62)=11.66, p=.001, \eta_{p}^{2}=.16$, and a marginal emotionality $\mathrm{x}$ distractor frequency interaction, $F(1,62)=.3 .84, p=.055, \eta_{p}^{2}=.058$. Importantly, all effects were qualified by a three way emotionality $\mathrm{x}$ distractor presence $\mathrm{x}$ distractor frequency interaction, $F(1,62)=11.81, \mathrm{p}=.001, \eta_{p}{ }^{2}=.16$ (see Figure 6 ).

To follow-up this three way interaction data was split by emotionality (i.e., negative and neutral blocks were analysed separately). For neutral blocks, no main effects or interactions were significant. For negative blocks, there were marginal main effects of distractor presence, $F(1,62)=3.69, p=.059, \eta_{p}^{2}=.06$, and distractor frequency, $F(1,62)=$ $3.63, p=.061, \eta_{p}{ }^{2}=.06$, that were qualified by a distractor presence $\mathrm{x}$ distractor frequency interaction, $F(1,62)=18.26, p=.059, \eta_{p}{ }^{2}=.23$. As shown in Figure 6, accuracy was lower on distractor-present than distractor-absent trials in the low distractor frequency condition, $t(31)=3.65, p=.001, d=.78$. This pattern of data is consistent with an emotional distraction effect (i.e., performance becomes worse) under reactive control conditions. Curiously, as shown in Figure 6, accuracy was higher on distractor-present than distractor-absent trials in the high distractor frequency condition, $t(31)=2.23, p=.033, d=.42$. This could suggest that the marginal emotional distraction effect (in RTs) in the high distractor frequency condition is due to a speed-accuracy tradeoff (i.e., to compensate for increased accuracy, responses were slower). Critically, results do not suggest that the emotional distraction effect observed in the low distractor frequency condition was due to a speed-accuracy tradeoff. 


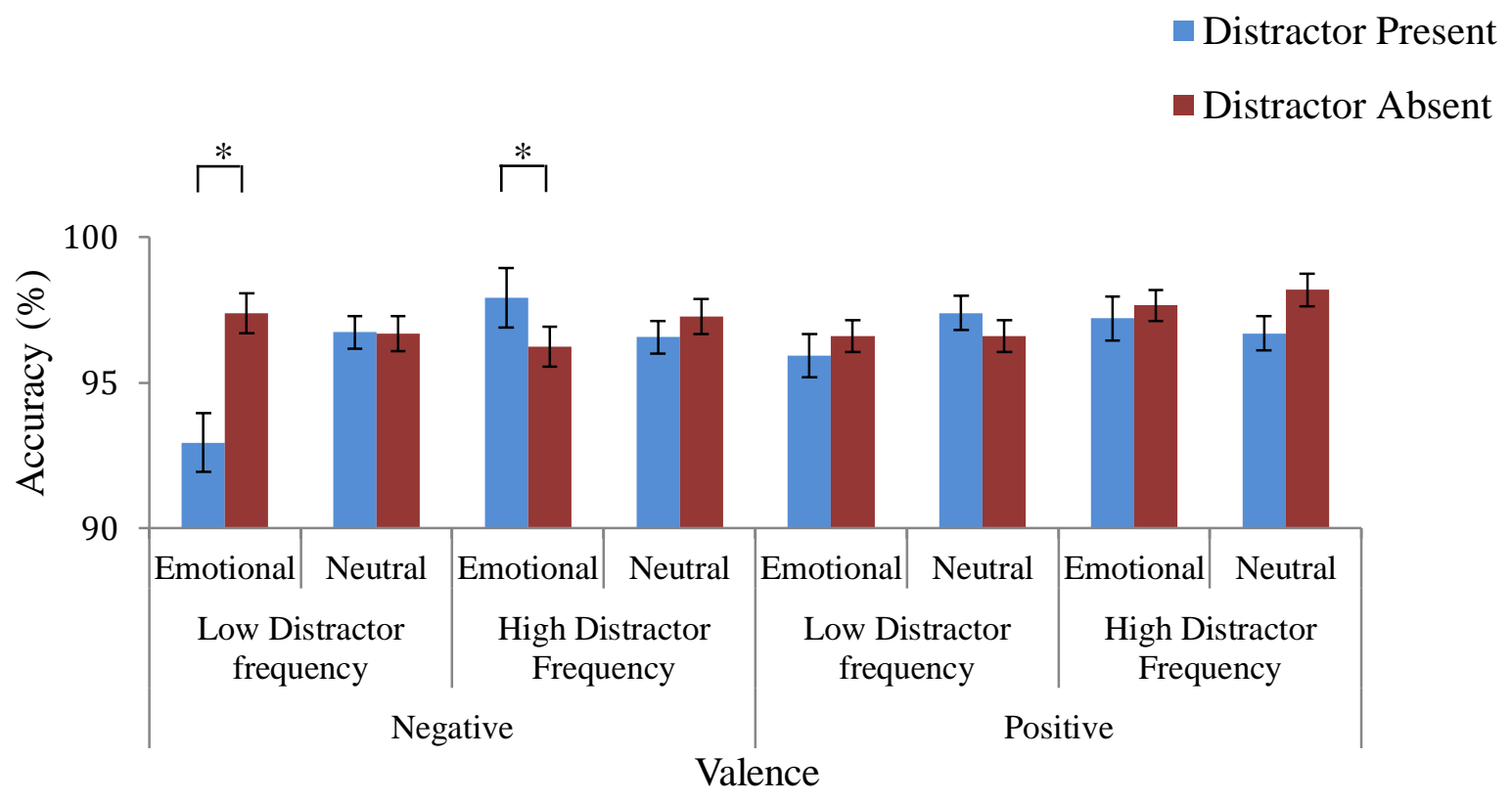

Figure 6. Mean accuracy (\%) on the distraction task in Experiment 1. Accuracy was lower on negative distractor-present, than negative distractor-absent trials in the low distractor frequency condition. In the high distractor frequency condition accuracy was higher on distractor-present, than distractor-absent negative trials.

Note. Bars represent standard errors of the mean

Note. $*=p<.05$

\section{Alternative Accounts.}

Although findings suggest that proactive mechanisms can effectively control emotional distraction, some alternative explanations of the results should be considered. First, the pronounced decrease in emotional distraction under high distractor frequency conditions could be due to habituation to the 'emotionality' of images. In the high distractor frequency condition, the 12 emotional distractors (of a given valence) were presented 6 times each (i.e., 72 presentations in total), whereas in the low distractor frequency condition the 12 distractors were only presented twice each (i.e., 24 presentations in total). Previous research has shown that repeated presentations of emotional information can lead to habituation of neural responses (in emotional processing areas) to its emotionality (e.g., Breiter et al., 1996; Feinstein, Goldin, Stein, Brown \& Paulus, 2002). It may be that participants show less emotional distraction under high distractor frequency conditions because, over time, they habituate to repeated viewing of emotional images - that is, the emotional images may lose their 'emotionality'. If so, I would expect that the attenuation of emotional distraction in the proactive condition should not arise until participants had experienced multiple exposures to the images. That is, attenuation should not be present at the beginning of the experiment 
(when the images are still novel), but should instead develop over time. To examine this possibility, RT's for the first 24 distractor-present trials (which is equivalent to the number of distractors in the low distractor frequency condition), as well as the first 24 distractor-absent trials (which is equivalent to the number in the high distractor frequency condition), were extracted from blocks with emotional distractors in the first half of the experiment.

A 2 (distractor frequency: low, high) x 2 (distractor presence: present, absent) x 2 (valence: positive, negative) mixed ANOVA was conducted. Valence was a between subjects variable in this analysis, as participants saw either positive or negative distractors in the first half of the experiment. For mean RTs and standard deviations by distractor frequency and distractor presence see Table 5. A main effect of distractor presence, $F(1,60)=23.36, p$ $<.001, \eta_{p}^{2}=.28$, indicated that significant distraction was observed. Importantly, there was a two-way distractor presence $\mathrm{x}$ distractor frequency interaction, $F(1,60)=6.92, p=.011, \eta_{p}{ }^{2}$ $=.10$. As shown in Figure 7, more emotional distraction was observed under low distractor frequency conditions, $t(31)=4.63, p<.001, d=.69$, than under high distractor frequency conditions, $t(31)=1.91, p=.070, d=.22$, even though the absolute number of emotional images presented was equated across conditions. This finding indicates good control over emotional distraction from the start of the experiment in the high distractor frequency condition. A follow-up analysis on RTs in the high distractor frequency condition confirmed that mean RTs for the first 24 distractor-present trials did not differ significantly from mean RTs for the remaining trials in the first half of the experiment, $t(31)=.48, p=.635$. This further indicates that emotional distraction did not fade over the course of the experiment. Taken together, these results suggest that habituation cannot account for the attenuation of emotional distraction under high distractor frequency conditions. The effect is more likely due to effective proactive control over emotional distraction under high distractor frequency conditions. 
Table 5

Mean (SD) RTs (ms) for the first 24 distractor-present and distractor-absent trials in blocks with emotional distractors by distractor frequency, and distractor presence in Experiment 1.

Distractor Present Distractor Absent Distraction Index

\begin{tabular}{lccc} 
& M (SD) & M (SD) & M (SD) \\
\hline Low Distractor Frequency & $656(109)$ & $597(56)$ & $59(72)$ \\
High Distractor Frequency & $601(76)$ & $583(82)$ & $17(51)$
\end{tabular}

Note. Distraction Index $=\mathrm{RT}$ on distractor-present trials $-\mathrm{RT}$ on distractor-absent trials

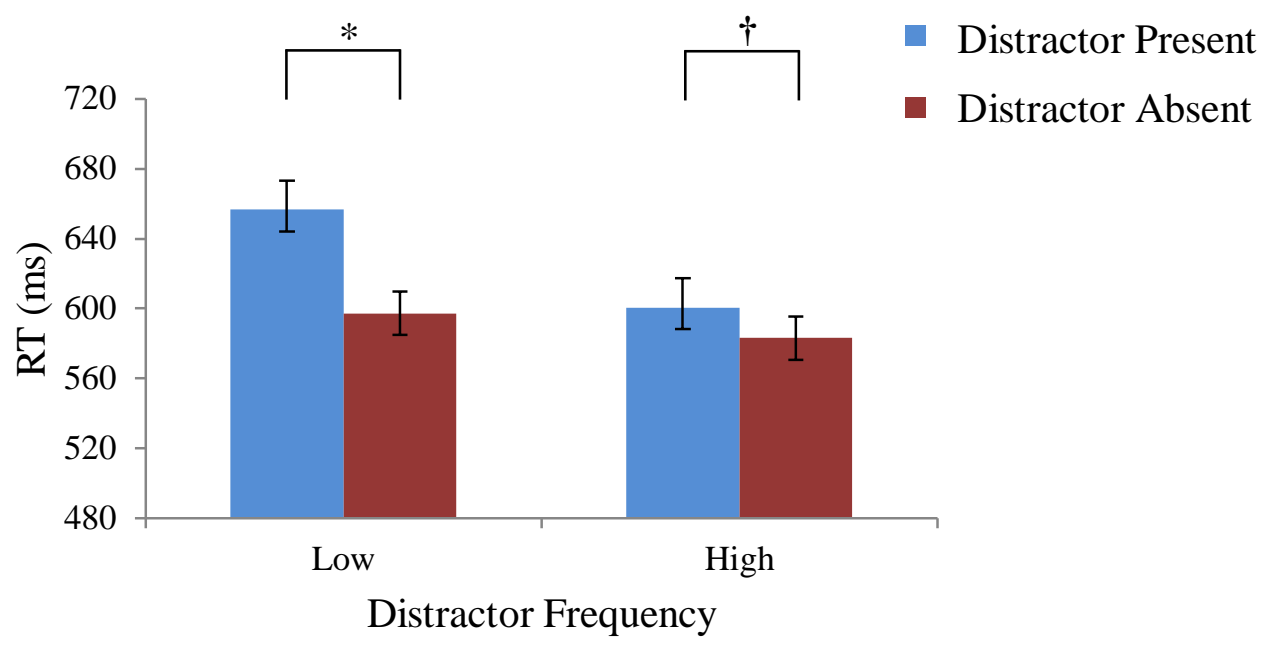

Figure 7. Mean RTs on the distraction task for emotional distractors, using only the first 24 distractor-present/distractor-absent trials, by distractor frequency condition in Experiment 1. Note. Bars represent standard errors of the mean

Note. $*=p<.05, \dagger=p<.1$

Second, one factor that could have artificially exaggerated the emotional, relative to neutral, distraction effects observed here is that the neutral image set used as distractors in the first half of the experiment was repeated in the second half, while the set of emotional distractors changed (to the opposite valence). It is possible that repeating the neutral set attenuated neutral distraction effects in the second half of the experiment, leading to an exaggerated overall emotional-neutral distraction difference across the experiment. If so, I would expect neutral distraction to be smaller in the second half of the experiment than in the first half, regardless of the valence in that half of the experiment. To examine whether this 
occurred, analyses were conducted on distraction indices (distractor-present - distractorabsent trials) to compare neutral and emotional distraction between each experiment half.

A 2 (emotionality: emotional, neutral) x 2 (experiment half: first, second) x 2(valence order: negative-positive, positive-negative) x 2 (distractor frequency: low, high) mixed ANOVA was conducted. Valence order was included as a between subjects variable in this analysis as participants saw either positive distractors in the first half of the experiment and negative distractors in the second half of the experiment, or vice versa. For means and standard deviations by distractor frequency, experiment half, valence order and emotionality see Table 6. As depicted in Figure 8, there was no main effect of experiment half, nor did experiment half interact with any other variables. This suggests that repeating the neutral images did not exaggerate the emotional distraction effect observed. Further, the absence of an effect of experiment half shows that distraction itself did not significantly change over the course of the experiment.

Table 6

Mean (SD) distraction indices (RT on distractor-present trials - RT on distractor-absent trials) by distractor frequency, experiment half, valence order, and emotionality in Experiment 1.

\begin{tabular}{lcccc}
\hline & \multicolumn{2}{c}{ Low Distractor Frequency } & \multicolumn{2}{c}{ High Distractor Frequency } \\
& $\begin{array}{c}\text { First Half } \\
\text { M (SD) }\end{array}$ & $\begin{array}{c}\text { Second Half } \\
\text { M (SD) }\end{array}$ & $\begin{array}{c}\text { First Half } \\
\text { M (SD) }\end{array}$ & $\begin{array}{c}\text { Second Half } \\
\text { M (SD) }\end{array}$ \\
\hline$\underline{\text { Positive-Negative }}$ & & & & \\
Emotional & $43(53)$ & $57(74)$ & $10(38)$ & $5(29)$ \\
Neutral & $17(28)$ & $18(35)$ & $0(45)$ & $-9(33)$ \\
Negative-Positive & & & & $19(28)$ \\
Emotional & $79(92)$ & $82(94)$ & $2(38)$ & $7(29)$ \\
Neutral & $37(61)$ & $18(40)$ & & \\
\hline
\end{tabular}




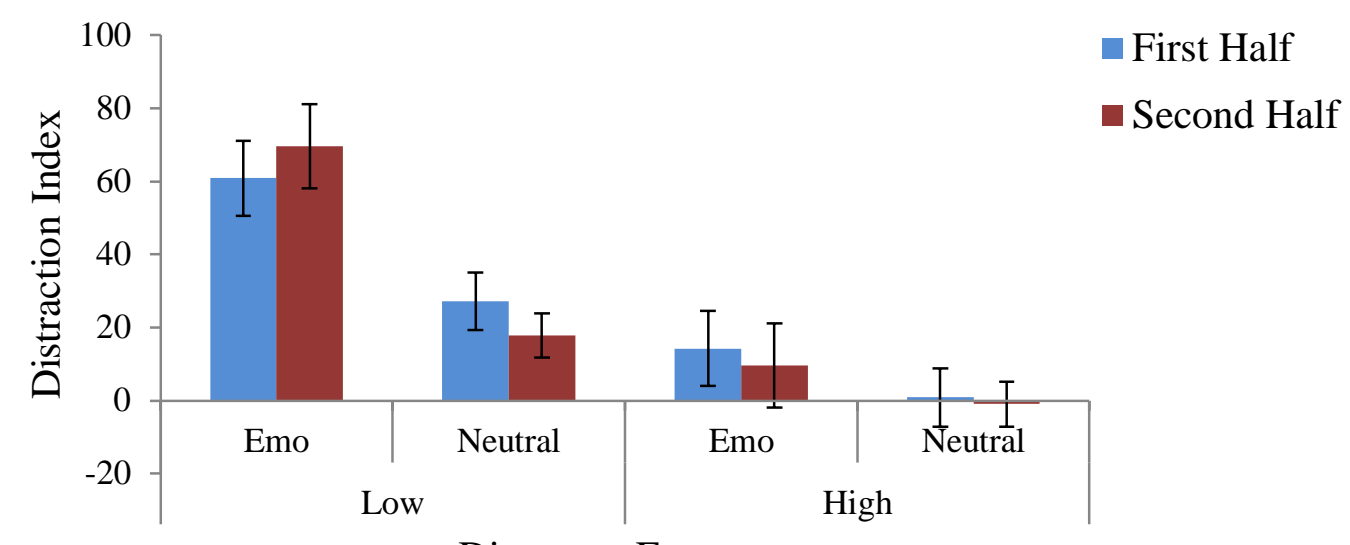

Figure 8. Mean distraction index (RT on distractor-present - RT on distractor-absent trials) by experiment half in Experiment 1.

Note. Bars represent standard errors of the mean

Note. ${ }^{*}=p<.05, \dagger=p<.1$

\section{Summary}

In Experiment 1 I determined whether emotional distractors could be effectively controlled using proactive control strategies. As predicted, more distraction occurred under low, relative to high, distractor frequency conditions, suggesting that proactive control was effectively evoked in the high distractor frequency condition. In line with a typical emotional distraction effect (e.g., Fox et al., 2012; Grimshaw et al., in prep.; Yates et al., 2010), irrelevant emotional images (both positive and negative) produced more distraction than irrelevant neutral images. Importantly, emotional distraction (compared to neutral distraction) was more pronounced under low distractor frequency conditions, or (put another way) was markedly reduced under high distractor frequency conditions (see Figure 5). Follow-up analyses indicated that this attenuation of emotional distraction could not be attributed to image repetition. Findings suggest that proactive control processes are able to control emotional (both positive and negative) distractions almost as effectively as neutral distractions. These findings do not support accounts which argue that emotional attention is independent of top-down control systems (e.g., Brosch et al., 2011; Viviani, 2013; Vuilleumier, 2005), but rather support the argument that emotional attention can be modulated by top-down control systems (e.g., Pessoa, 2005; Pessoa et al., 2002; Pourtois et al., 2013). Further, findings extend those of Padmala and Pessoa (2014) who found that proactive strategies can control negative distraction as effectively as neutral distraction when distractors are presented in attended locations. Interestingly, a small emotional distraction effect was still observed in the high distractor frequency condition, suggesting that even 
when proactive control is engaged, emotional characteristics of distractors are still processed. It may be that a healthy cognitive system always maintains a background goal to process emotional stimuli to a certain extent due to their survival relevance (LeDoux, 1996). Alternatively, it could be that including a stronger incentive to use proactive control (over and above distractor frequency) would eliminate the remaining emotional distraction.

One alternative account of the interaction between emotionality and distractor frequency - i.e., the pronounced decrease in emotional, compared to neutral, distraction under high distractor frequency conditions - is that it could reflect a floor effect for neutral distraction. No neutral distraction was observed in the high distractor frequency condition; RTs did not differ between distractor-present and distractor-absent trials. As it would be impossible for neutral distraction to decrease as much as emotional distraction from low to high distractor frequency conditions, the interaction could be explained by neutral distraction reaching floor. To test if this alternative explanation accounts for the pattern of results, a future study might need to use intermediate distractor probabilities (i.e., less than $75 \%$ ) that still shift control towards proactive strategies, but produce measurable neutral distraction.

Experiment 1 established that proactive mechanisms can control entirely irrelevant emotional distraction almost as effectively as irrelevant neutral distraction, suggesting that proactive mechanisms are able to limit the attentional gain attached to emotional stimuli. However, the specific mechanisms by which proactive control reduces distraction from irrelevant emotional stimuli is not clear. It is possible that, under proactive control conditions, emotional images receive prioritisated attention at early stages of processing, but are then prevented from interrupting the goal and causing distraction at later stages of processing. Alternatively, it is possible that proactive control reduces early perceptual prioritisation of emotional images. In Experiment 2, I used ERP measures of emotional processing to address this question of mechanism.

\section{Experiment 2}

In Experiment 2 I used event related potentials (ERPs) to examine the mechanisms by which proactive control reduces emotional distraction from irrelevant images. ERPs reflect neural responses to a particular event - e.g., the onset of a stimulus. ERPs can be extracted from electroencephalogram (EEG) signals that are passively recorded from electrodes on the scalp. An EEG signal at a given electrode represents a summation of changes in the voltage of post-synaptic potentials (Luck, 2005). ERPs can be extracted by recording EEG in response to a trigger (e.g., time-locked to the onset of a picture) across a number of trials. By averaging the time-locked EEG signal across trials, noise in the signal cancels out, and the 
remaining signal represents the neural response to the trigger - an ERP. Measuring ERPs enables fine-grained analysis of the temporal processing of stimuli, and so is particularly useful for examining when something happens.

Two ERP components that are commonly used as indices of emotional processing are the early posterior negativity (EPN) and late positive potential (LPP). The EPN is a relatively early component, occurring approximately 200 to $300 \mathrm{~ms}$ after stimulus onset and is thought to reflect early recruitment of attentional resources for perceptual processing of a stimulus (Hajcak et al., 2012; Junghöfer, Bradley \& Elbert, 2001; Schupp, Flaisch, Stockburger \& Junghöfer, 2006). The LPP is a relatively late component (beginning approximately 300 to $500 \mathrm{~ms}$ after stimulus onset) and is thought to reflect sustained attention to a stimulus (Hajcak et al, 2012; Schupp et al., 2006a; Wiens \& Syrjänen, 2013). Both of these components are sensitive to the emotionality of stimuli - that is, they are more pronounced for emotional compared to neutral stimuli (Hajcak et al., 2012).

\section{The Early Posterior Negativity (EPN)}

The EPN belongs to the $\mathrm{N} 2$ range of components. It manifests as early as $150 \mathrm{~ms}$ post stimulus onset, and is maximal approximately 200 to 300ms post stimulus onset (Hajcak et al., 2012; Junhöfer et al., 2001; Schupp et al., 2006a). The EPN is thought to index an early attentional response. Greater EPN amplitudes are argued to reflect increased allocation of attentional resources towards the perceptual processing of a stimulus (Hajcak et al., 2012; Schupp et al., 2006a; Wiens \& Syrjänen, 2013). Indeed, the EPN has been associated with increased visual processing of stimuli (De Cesarei \& Codispoti, 2006; Foti, Hajcak \& Dien, 2009). The EPN manifests as a relative negativity (often a reduction in a positivity) maximal over temporo-occipital sites in response to emotional compared to neutral information (Hajcak et al., 2012). The EPN is sensitive to both negative and positive information and shows greater potentiation for higher arousal emotional stimuli (Junghöfer et al., 2001; Schupp, Junghöfer, Weike \& Hamm, 2003a, 2004a; Olofsson, Nordin, Sequeira \& Polich, 2008).

Modulation of the EPN by emotional stimuli is robust (see Hajcak et al, 2012). The EPN has been observed using presentation durations ranging from less than $100 \mathrm{~ms}$ (e.g., Herbert, Junghofer \& Kissler, 2008) to more than 1500ms (e.g., Weinberg \& Hajcak, 2010). Further, the EPN has been observed across a range of paradigms (see Hajak et al., 2012; Olofsson et al., 2008), using various stimuli, including words (e.g., Scott, O’Donnell, Leuthold \& Sereno, 2009), scenes (e.g., Schupp, Junghöfer, Weike \& Hamm, 2003b), faces 
(e.g., Schupp et al., 2004b), and even hand gestures (e.g., Flaisch, Häcker, Renner \& Schupp, 2011). Although the EPN has been observed for emotional stimuli in a range of experimental conditions, it can be modulated by both perceptual and attentional manipulations. For example, reducing the size of a stimulus reduces emotional modulation of the EPN (e.g., De Cesarei \& Codisopti, 2006). Similarly, diverting attention away from an emotional stimulus also reduces the magnitude of the EPN (e.g., Norberg \& Wiens, 2013; Schupp et al., 2007; Wiens, Sand, Norberg \& Andersson, 2011). Importantly, the EPN can be used as an early index of attentional resources that have been recruited to perceptually process an emotional stimulus.

\section{The Late Positive Potential (LPP)}

The LPP manifests as a relative positivity over centro-parietal sites beginning between 300 and 500ms post-stimulus onset and continues for a sustained period (Hajcak et al., 2012; Hajcak \& Nieuwenhuis, 2006; Weinberg \& Hajcak, 2010). The LPP is thought to index sustained attention to stimuli, with higher amplitudes (more positivity) reflecting an increase in sustained attention (Hajcak et al, 2012; Schupp et al., 2006a; Wiens \& Syrjänen, 2013). Potentiated LPPs have been observed for both positive and negative stimuli, relative to neutral stimuli (e.g., Cutherbert, Schupp, Bradley, Birbaumer \& Lang, 2000; Schupp et al., 2000; Weinberg \& Hajcak, 2010). Further, like the EPN, the LPP is sensitive to arousal, with more arousing emotional stimuli producing more pronounced potentiation of the LPP (Schupp et al., 2000). Indeed, LPP amplitudes have been found to increase in accordance with subjective ratings of arousal for a given stimulus (Cuthbert et al., 2000; Weinberg \& Hajcak, 2010).

Modulation of the LPP by emotional stimuli is also robust (see Hajcak et al., 2012). It has been observed using both short (e.g., 200ms; Syrjänen \& Wiens, 2013) and long (e.g., 6seconds; Cuthbert et al., 2000) presentation durations, and across both passive viewing paradigms (e.g., Weinberg \& Hajcak. 2010), and paradigms that involve engagement in tasks (e.g., Syrjänen \& Wiens, 2013). The LPP is long lasting, and continues after stimulus offset (Hajcak \& Olvet, 2008). Similar to the EPN, the LPP can be affected by manipulations of attention. Reducing the amount of attention paid to a stimulus has been found to reduce the amplitude of the LPP it elicits (e.g., Wiens, Molapour, Overfeld \& Sand, 2012). However, the LPP is more resistant to perceptual changes such as stimulus size (De Cesarei \& Codispoti, 2006). The LPP is also affected by manipulations of motivational relevance (Hajcak et al., 2012). For example, LPP amplitudes are attenuated when participants are instructed to 
reappraise emotional stimuli (e.g., Hajcak \& Nieuwenhuis, 2006). Importantly, the LPP can be used as an index of sustained attention to emotional stimuli at later stages of processing.

\section{The Current Experiment}

In Experiment 2 participants completed a distraction task similar to that in Experiment 1, and EEG was recorded concurrently. From EEG recordings, ERPs in response to images (i.e., the distractors) were extracted. The EPN and LPP were examined in order to assess when proactive control reduces distraction by emotional information. As the EPN and LPP index different stages in the time-course of emotional processing, examining both components in conjunction can reveal whether emotion is processed at both earlier and later stages of processing, earlier stages only, or neither. By association it is possible to infer when emotional characteristics of stimuli are not being processed. Using the LPP and EPN, I can determine when proactive control minimises distraction by irrelevant emotional distractors. Does proactive control work by reducing early attentional recruitment for perceptual processing of emotional distractors, or rather by preventing sustained attention to emotional stimuli so that they do not disrupt our goal?

Under reactive control conditions (i.e., low distractor frequency), in which pronounced emotional distraction was observed in Experiment 1, it was expected that typical emotional potentiation of the EPN and LPP would be observed; emotional images should produce more positive LPP amplitudes, and more negative EPN amplitudes, compared to neutral images. Importantly, if proactive control minimises emotional distraction by preventing early allocation of attention to emotional stimuli for perceptual processing, then under high distractor frequency emotional modulation of both the EPN and LPP should be attenuated or even eliminated. Alternatively, if proactive control minimises emotional distraction by decreasing sustained attention to emotional stimuli, but emotional stimuli still receive prioritised processing at earlier stages, then the LPP should be attenuated or eliminated under conditions of high distractor frequency, but emotional modulation of the EPN should still be observed.

\section{Anterior N2}

Results from Experiment 1 clearly indicate that manipulating distractor frequency affects cognitive control. As such, an exploratory analysis was conducted on a third ERP component - the anterior N2 (also known as the frontal N2, the go no/go N2, and the frontocentral N2) - to determine if it would index the cognitive control manipulation. The anterior $\mathrm{N} 2$ is a negative-going fronto-central component peaking approximately 200 to $350 \mathrm{~ms}$ after stimulus onset (Henderson, 2010; Nieuwenhuis et al., 2003). The anterior N2 has primarily 
been studied in paradigms requiring inhibition of a prepotent response - e.g., go no/go tasks (Eimer, 1993; Jodo \& Kayama, 1992; Kok, 1986; Pfefferbaum, Ford, Weller, \& Kopell, 1985). Such paradigms, by their design, evoke reactive cognitive control - for example, in a typical go no/go task, participants respond on frequent 'go' signals, and have to withhold response on infrequent 'no/go' signals. On no/go trials, participants have to reactively control their prepotent 'go' response. On these trials, a larger (i.e., more negative) anterior N2 is observed (Eimer, 1993; Jodo \& Kayama, 1992; Kok, 1986; Pfefferbaum et al., 1985). Further, the amplitude of the anterior N2 is affected by stimulus frequency manipulations, with more potentiation for less frequent signals (Nieuwenhuis et al., 2003) - i.e., when reactive control is more heavily relied upon. Importantly, the anterior N2 does not specifically index response inhibition, but extends to more general cognitive control over conflict (e.g., Botvinick Braver, Barch, Carter \& Cohen, 2001; Falkenstein, Hoormann \& Hohnsbein, 1999; Nieuwenhuis et al., 2003; Nieuwenhuis, Yeung \& Cohen, 2004).

The anterior N2 was examined in Experiment 2 as it could provide a useful index of effective manipulation of cognitive control. The anterior N2 was expected to be larger under low distractor frequency (which is assumed to evoke reactive control), compared to high distractor frequency.

\section{Method}

\section{Participants}

Seventy right-handed women aged 18 to $30(M=20.10, S D=2.31)$ with normal or corrected-to-normal vision were recruited. Participants were first year psychology undergraduate students from Victoria University of Wellington who received course credit for their participation, as well as members of the community, who received vouchers. Participants reported no neurological issues, as well as no past or current treatment for depression or anxiety. Participants were randomly assigned to the low or high distractor frequency condition. Written informed consent was attained prior to participation. This study was approved by the Human Ethics Committee of the School of Psychology, Victoria University of Wellington (Wellington, New Zealand).

\section{Materials}

\section{Distraction Task.}

The distraction task used in Experiment 1 was slightly modified for Experiment 2. The trial procedure remained identical. However, trial numbers were increased in order to attain stable ERP measures. Participants completed a total of 720 trials across nine blocks (80 trials per block). As in Experiment 1, distractors were presented on either 75\% (60 trials per 
block) or $25 \%$ (20 trials per block) of trials - manipulated between subjects. In Experiment 1 valence (negative, positive) and emotionality (emotional, neutral) were crossed factorially. However in Experiment 2, these variables were combined to create one variable, valence, with three levels: negative, positive and neutral ${ }^{4}$. Valence was blocked such that participants completed blocks in one of the following orders: negative-negative-negative/neutral-neutralneutral/positive-positive-positive or positive-positive-positive/neutral-neutralneutral/negative-negative-negative. Neutral blocks were always completed in the middle to reduce any carry over effects between negative and positive blocks. Between each set of blocks (i.e., after the third and sixth block) the experimenter checked electrode impedances.

Before beginning the task, participants completed 24 practice trials. Unlike Experiment 1, practice trials contained distractors that were presented at the same frequency as participants' assigned condition (i.e., $75 \%$ or $25 \%$ ). This was done in order to evoke proactive or reactive control strategies before participants began the first block of trials. Distractors for the practice trials were an independent set of 6 neutral images taken from the IAPS (Lang et al., 2008) ${ }^{5}$.

\section{Questionnaires.}

The same questionnaires as in Experiment 1 were administered - the Mini-MASQ (Clark \& Watson, 1995) and the Attentional Control Scale (Derryberry \& Reed, 2002).

\section{Procedure}

Participants took part in the experiment individually, and each session took approximately one and a half hours. EEG recordings took place in a dimly lit, electricallyshielded chamber. Participants completed the same tasks as in Experiment 1, in the same order: 1) distraction task; and 2) questionnaires (Mini-MASQ followed by the Attentional Control Scale). Tasks were completed on a Dell Precision T1600 computer, with a 23" Alienware 2310 LCD monitor running at a vertical refresh rate of $60 \mathrm{~Hz}$. An approximate viewing distance of $57 \mathrm{~cm}$ was maintained using a chinrest.

\section{EEG Recording and Analysis}

EEG was recorded during the distraction task using a Lycra Quick-Cap

\footnotetext{
${ }^{4}$ The experiment design was changed from an emotionality by valence design, to just one variable (valence) with three levels, as this reduced the total number of trials needed in the experiment. This allowed for the experiment to be completed in the allotted time, despite increasing the number of trials per condition in order to get a stable ERP response.
}

${ }^{5}$ IAPS images numbers used for practice trials were: $2192 ; 2235 ; 2382 ; 2396 ; 2579$; and 2890. The mean arousal rating for this set of images is $3.41(S D=2.03)$, and the mean valence rating is $5.29(S D=1.48)$. Ratings were taken from Lang et al. (2008). 
(Compumedics NeuroMedical Supplies) embedded with $\mathrm{Ag} / \mathrm{AgCl}$ electrodes. EEG was recorded from 28 scalp sites (FP1, FP2, F7, F3, FZ, F4, F8, FT7, FC3, FC4, FT8, T7, C3, CZ, C4, T8, TP7, CP3, CP4, TP8, P7, P3, PZ, P4, P8, O1, OZ, and O2, according to the modified 10-20 system American Electroencephalographic Society, 1994). Electrodes were also placed on the mastoid bones behind the left and right ear. Additionally, blinks and eye movements were measured by recording electrooculogram (EOG) from electrodes placed above (vertical EOG upper; VEOU) and below (vertical EOG lower; VEOU) the left eye, and on the outer canthus of each eye (horizontal EOG left; HEOL, and horizontal EOG right; HEOR). Online recordings were referenced to the left mastoid. Impedances were kept below $10 \mathrm{k} \Omega$ and checked periodically throughout the experiment. The EEG signal was amplified with Professional BrainAmps and digitized at a sampling rate of $500 \mathrm{~Hz}$ with Brain-Vision Recorder (Brain Products, Gilching, Germany). Data was filtered online with a highpass filter of $0.02 \mathrm{~Hz}$.

Data were analysed using Brain-vision Analyzer 2.0 (Brain Products, Gilching, Germany). For LPP and anterior N2 analyses, data were re-referenced offline to the algebraic average of electrodes at the left and right mastoids. For EPN analyses, data were rereferenced offline to the algebraic average of all scalp electrodes. The EPN is known to manifest more reliably under average scalp reference montages, while the LPP manifests more reliably under average mastoid reference montages (Hajcak et al., 2012). Vertical EOG (VEOG) and horizontal EOG (HEOG) channels were created by calculating the difference between VEOL and VEOU, and HEOR and HEOL, respectively. Data was filtered with a low cut-off of 0.01 , a high cut-off of $30 \mathrm{~Hz}$, and a notch filter at $50 \mathrm{~Hz}$ using a zero phaseshift Butterworth filter (12 dB/oct). It was then segmented into epochs for correct distractorpresent trials, so as to contrast the neural responses on trials with emotional vs. neutral images as a function of distractor frequency. For the LPP, epochs were $1200 \mathrm{~ms}$, beginning $200 \mathrm{~ms}$ before stimulus onset and ending 1000ms after stimulus onset. For the EPN and anterior N2, epochs were $650 \mathrm{~ms}$, beginning $200 \mathrm{~ms}$ before stimulus onset and ending $450 \mathrm{~ms}$ after stimulus onset. Segments were baseline corrected by subtracting the average signal $200 \mathrm{~ms}$ prior to stimulus onset. Ocular artifacts (i.e., eye movements and blinks) were corrected using the method of Gratton and Coles (Gratton, Coles \& Donchin, 1983).

Segments were re-baselined after ocular correction. To ensure that participants saw the visual stimulus, segments containing eye blinks (a change exceeding $100 \mu \mathrm{V}$ measured at VEOG across any $200 \mathrm{~ms}$ period) between $-200 \mathrm{~ms}$ and $200 \mathrm{~ms}$ were removed. Although the stimulus was only presented for $100 \mathrm{~ms}(0-100 \mathrm{~ms})$ a conservative criterion was chosen to ensure that 
the stimulus was visually processed. Two participants' data had a noisy VEOU channel for three blocks of trials. For these blocks, blinks were identified and rejected based on a change exceeding $100 \mu \mathrm{V}$ measured at FP1 or FP2 across any 200ms period, rather than using the VEOG channel (see Grimshaw, Foster \& Corballis, 2014). Segments with other recording artifacts (a voltage exceeding $+/-100 \mu \mathrm{V}$ ) at electrodes of interest (as outlined below for each component and depicted in Figure 9) were removed.

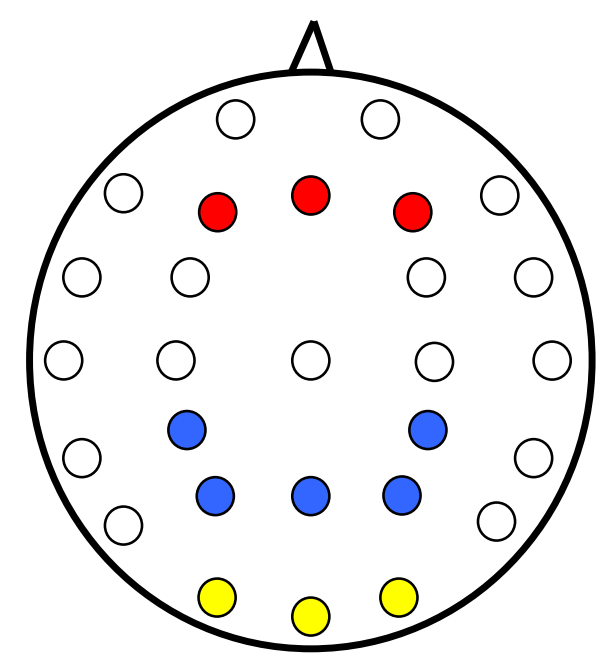

Figure 9. Scalp location of the electrodes of interest. Red represents electrodes for anterior N2 analyses (F3, FZ, F4), blue represents electrodes for LPP analyses (PZ, P3, P4, CP3, $\mathrm{CP} 4)$, and yellow represents electrodes for EPN analyses (O1, OZ, O2)

\section{LPP.}

The LPP is known to manifest at centro-parietal sites (Hajcak et al., 2012; Hajcak \& Nieuwenhuis, 2006; Weinberg \& Hajcak, 2010) so it was measured from electrode sites PZ, P3, P4, CP3, CP4 (see Figure 9). The LPP amplitude was defined as the mean activity ( $\mu \mathrm{V}$ ) in the interval from $400 \mathrm{~ms}$ to $800 \mathrm{~ms}$ after stimulus onset. This window is fairly typical for an LPP (e.g., Schupp et al., 2000 - 350ms to 750ms; Brown, Goodman \& Inzlicht, 2012 500ms to $900 \mathrm{~ms}$; Wiens \& Syrjänen, 2013 - 400ms to 700ms), and was chosen based on visual inspection of the data ${ }^{6}$. The rejection criteria (as described above) for LPP analyses led to the rejection of $6.22 \%$ of trials.

\section{EPN.}

The EPN is maximal at temporo-occipital electrode sites (Foti et al., 2009; Junghöfer et al., 2001; Schupp et al., 2006a). Based on previous studies that recorded from relatively

\footnotetext{
${ }^{6}$ The LPP begins after the P3 component (a prominent positive deflection in the waveform typically between 300 and 400ms; Hajcak et al., 2012). This makes it easily identifiable via visual inspection of waveforms.
} 
few electrode sites (Holmes, Nielsen, Tipper \& Green, 2009 - 64 channels; Wronka \& Walentowska, 2011 - 32 channels), the EPN was measured from occipital electrode sites: O1, OZ, O2 (see Figure 9). The EPN amplitude was defined as the mean activity ( $\mu \mathrm{V}$ ) from $180 \mathrm{~ms}$ to $260 \mathrm{~ms}$ post stimulus onset. This time window was chosen by first collapsing data across distractor frequency conditions for emotional and neutral trials separately. Next, the biggest emotional-neutral difference collapsed across occipital electrode sites $(\mathrm{O} 1, \mathrm{OZ}, \mathrm{O} 2)$ in the time window between 200 and $300 \mathrm{~ms}$ (the time window in which a typical EPN is observed; Hajcak et al., 2012; Oloffson et al., 2008) was identified. Finally, an 80ms time window (a time window comparable to other studies; e.g., Holmes et al., 2009; Weinberg \& Hajcak, 2010; Wiens et al., 2012) around this peak difference was chosen. The rejection criteria (as described above) for EPN analyses led to the rejection of $6.04 \%$ of trials.

\section{Anterior N2.}

The anterior $\mathrm{N} 2$ is known to be maximal at fronto-central electrode sites (Nieuwenhuis et al., 2003; Pandey et al., 2012) so it was measured from electrode sites F3, FZ, F4 (see Figure 9). While previous studies often use peak analysis methods to examine the anterior N2 (e.g., Nieuwenhuis et al., 2003; Pandey et al., 2012), I used a small window around the $\mathrm{N} 2$ peak. This is because there were unequal trial numbers across conditions (low distractor frequency $=60$ trials per valence, high distractor frequency $=180$ trials per valence). Unequal trial numbers can bias data when using peak analysis methods as conditions with fewer trials have more extreme peaks (Luck, 2010). However, unequal trial numbers do not bias data when using mean activity across a time window because extreme peaks are equally likely to go in either direction (i.e., higher or lower) leading to this additional noise being cancelled out (Luck, 2010). The anterior N2 amplitude was defined as mean activity $(\mu \mathrm{V})$ between $225 \mathrm{~ms}$ and $255 \mathrm{~ms}$. This window was chosen by collapsing trials across all conditions, identifying the biggest negative peak from $200 \mathrm{~ms}$ to $350 \mathrm{~ms}$ (i.e., the time in which a typical anterior N2 manifests; Nieuwenhuis et al., 2003), and then choosing a $30 \mathrm{~ms}$ window around this peak. A relatively small window of $30 \mathrm{~ms}$ was chosen to ensure that analyses focused on the peaking section of the anterior N2. The rejection criteria (as described above) for anterior N2 analyses led to the rejection of $6.17 \%$ of trials.

\section{Design}

The independent variables were distractor frequency (high, low; between subjects), distractor valence (negative, neutral, positive; within subjects), and distractor presence (present, absent; within subjects). The dependent variables were RT and accuracy on the 
letter search task, and amplitudes $(\mu \mathrm{V})$ for the three ERP components of interest: EPN, LPP and anterior $\mathrm{N} 2$.

\section{Results and Discussion}

\section{Questionnaires}

Question skipping rates were very low (Mini-MASQ $=.0045 \%$ of questions, Attentional Control Scale $=.0015 \%$ of questions). As in Experiment 1, questionnaires were used to ensure that participants in the low and high distractor frequency condition did not differ on symptoms of depression, anxiety or attentional control as these factors could be expected to affect distraction. For mean questionnaire scores and standard deviations by distractor frequency condition see Table 7. Scores on the Attentional Control Scale did not significantly differ between participants in the low and high distractor frequency conditions, $t(68)=1.53, p=.132$. Total Mini-MASQ scores also did not significantly differ between the low and high distractor frequency conditions, $t(68)=1.06, p=.29$ Further, participants in each distractor frequency condition did not significantly differ on scores on the three subscales of the Mini-MASQ: general distress, $t(68)=.61, p=.544$; anxious arousal, $t(68)=$ $.85, p=.401 ;$ and anhedonic depression, $t(68)=.95, p=.348$.

Table 7

Mean (SD) questionnaire scores by distractor frequency in Experiment 2.

\begin{tabular}{lcc}
\hline & $\begin{array}{c}\text { Low Distractor } \\
\text { Frequency } \\
\text { M (SD) }\end{array}$ & $\begin{array}{c}\text { High Distractor } \\
\text { Frequency } \\
\text { M (SD) }\end{array}$ \\
\hline Attentional Control Scale & $2.33(.44)$ & $2.48(.38)$ \\
Mini MASQ Total & $1.95(.53)$ & $2.07(.46)$ \\
Mini MASQ: general distress & $1.91(.76)$ & $2.01(.65)$ \\
Mini MASQ: anxious arousal & $1.53(.46)$ & $1.64(.59)$ \\
Mini MASQ: anhedonic Depression & $2.47(.76)$ & $2.63(.70)$ \\
\hline
\end{tabular}

Note. Scores range from 1 to 4 for the Attentional Control Scale, and from 1 to 5 for the Mini-MASQ, as well as the three Mini-MASQ subscales: general distress, anxious arousal, anhedonic depression.

\section{Distraction Task Exclusion Criteria}

Mean RTs and accuracy rates for the distraction task were calculated for each condition. The same behavioural exclusion criteria were applied as in Experiment 1 - only 
accurate responses longer than 200ms were included, and accuracy was required to be higher than $85 \%$. Two participants' data was removed due to low accuracy $(<85 \%)$ leaving 68 participants (low distractor frequency: $\mathrm{N}=34$; high distractor frequency: $\mathrm{N}=34$ ). For the remaining participants, the behavioural exclusion criteria led to the average removal of $4.33 \%$ of trials $(S D=2.57)$ per participant. Additionally, for the ERP analyses two participants' data was removed due to technical issues during recording, and three participants' data was removed as less than $75 \%$ of their trials remained after the ERP artifact rejection criteria was applied (as described in the EEG recording and analysis section above), leaving 63 participants (high distractor frequency: $\mathrm{N}=31$, low distractor frequency: $\mathrm{N}=32$ ).

\section{Behavioural Results}

Analyses were conducted on data from the 68 participants remaining after the behavioural exclusion criteria were applied. While valence and emotionality were two crossed variables in Experiment 1 (an emotionality x valence design), in Experiment 2 only one variable, valence, with three levels (positive, negative, neutral) was used. Based on the results from Experiment 1, two of the conditions (positive and negative) were expected to produce larger distraction effects than the third condition (neutral). Therefore quadratic effects were also examined. By always inserting 'neutral' as the middle variable in analyses, examining quadratic relationships allowed me to assess in a hypothesis driven manner whether emotional conditions differed from neutral conditions. Quadratic relationships were only examined for predicted RT effects (i.e., interactions involving valence). For all analyses, when Mauchly's test of sphericity was violated, Greenhouse-Geisser corrections were applied.

\section{Response Times.}

To examine distraction, RTs were analysed in a mixed 2 (distractor frequency: low, high) x 3 (valence: negative, neutral, positive) x 2 (distractor presence: present, absent) ANOVA. For mean RTs and standard deviations by distractor frequency, valence and distractor presence see Table 8 . A significant main effect of distractor presence, $F(1,66)=$ $46.60, p<.001, \eta_{p}{ }^{2}=.41$, indicated that there was a typical distraction effect - responses were slower on distractor-present than distractor-absent trials. RTs were marginally slower in the high compared to low distractor frequency condition, $F(1,66)=3.74, p=.057, \eta_{p}{ }^{2}=.05$. This slowing of RT in the high distractor frequency condition was unexpected, and not observed in Experiment 1. There was also a significant distractor presence by distractor frequency interaction, $F(1,66)=15.49, p<.001, \eta_{p}{ }^{2}=.19$, and a marginal main effect of valence $F(1.67,109.96)=2.72, p=.080, \eta_{p}{ }^{2}=.04$, which were both were qualified by the 
expected three way valence $\mathrm{x}$ distractor presence $\mathrm{x}$ distractor frequency interaction, $F(1.66$, $109.62)=3.63, p=.038, \eta_{p}^{2}=.05$.

Table 8

Mean (SD) RTs (ms) for the distraction task, for participants who met the behavioural inclusion criteria, by distractor frequency, valence and distractor presence in Experiment 2. Low Distractor Frequency High Distractor Frequency

Distractor Distractor Distraction Distractor Distractor Distraction Present Absent Index Present Absent Index

\begin{tabular}{lcccccc} 
Valence & M (SD) & M (SD) & M (SD) & M (SD) & M (SD) & M (SD) \\
\hline Negative & $581(78)$ & $553(64)$ & $28(28)$ & $605(85)$ & $599(92)$ & $6(27)$ \\
Neutral & $562(78)$ & $548(74)$ & $14(31)$ & $592(94)$ & $582(80)$ & $10(30)$ \\
Positive & $573(81)$ & $543(66)$ & $30(30)$ & $601(97)$ & $597(100)$ & $4(20)$
\end{tabular}

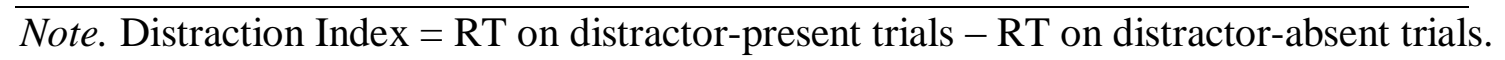

To further explore this three-way interaction data were split by distractor frequency. A 3 (valence: negative, neutral, positive) x 2 (distractor presence: present, absent) repeated measures ANOVA was conducted for low and high distractor frequency conditions separately. In the low distractor frequency condition, a typical distraction effect was observed; responses were slower on distractor-present compared to distractor-absent trials, $F(1,33)=43.15, p<.001, \eta_{p}{ }^{2}=.57$. Importantly, there was a predicted two-way valence $\mathrm{x}$ distractor presence interaction, $F(2,66)=4.40, p=.016, \eta_{p}{ }^{2}=.12$. As shown in Figure 10, an emotional distraction effect was observed; there was more distraction from positive images (31ms of distraction), $t(33)=5.93, p<.001, d=.41$, and negative images ( $28 \mathrm{~ms}$ of distraction), $t(33)=5.96, p<.001, d=.39$, than neutral images ( $14 \mathrm{~ms}$ of distraction), $t(33)=$ $2.73, p=.010, d=.18$. This replicates the findings of Experiment 1 , and suggests that when reactive control is engaged, emotional distractors are more potent than neutral distractors.

In the high distractor frequency condition, images still produced distraction; responses were slower on distractor-present compared to distractor-absent trials, $F(1,33)=6.35, p$ $=.017, \eta_{p}{ }^{2}=.16$. However, distraction levels were very low $(7 \mathrm{~ms}$ as compared to $24 \mathrm{~ms}$ that was observed under low distractor frequency conditions) indicating that proactive control was effectively engaged. Importantly, distractor presence did not interact with valence, suggesting that emotional images were no more distracting than neutral images. This supports findings 
from Experiment 1, and suggests that proactive mechanisms control emotional distraction as effectively as neutral distraction.

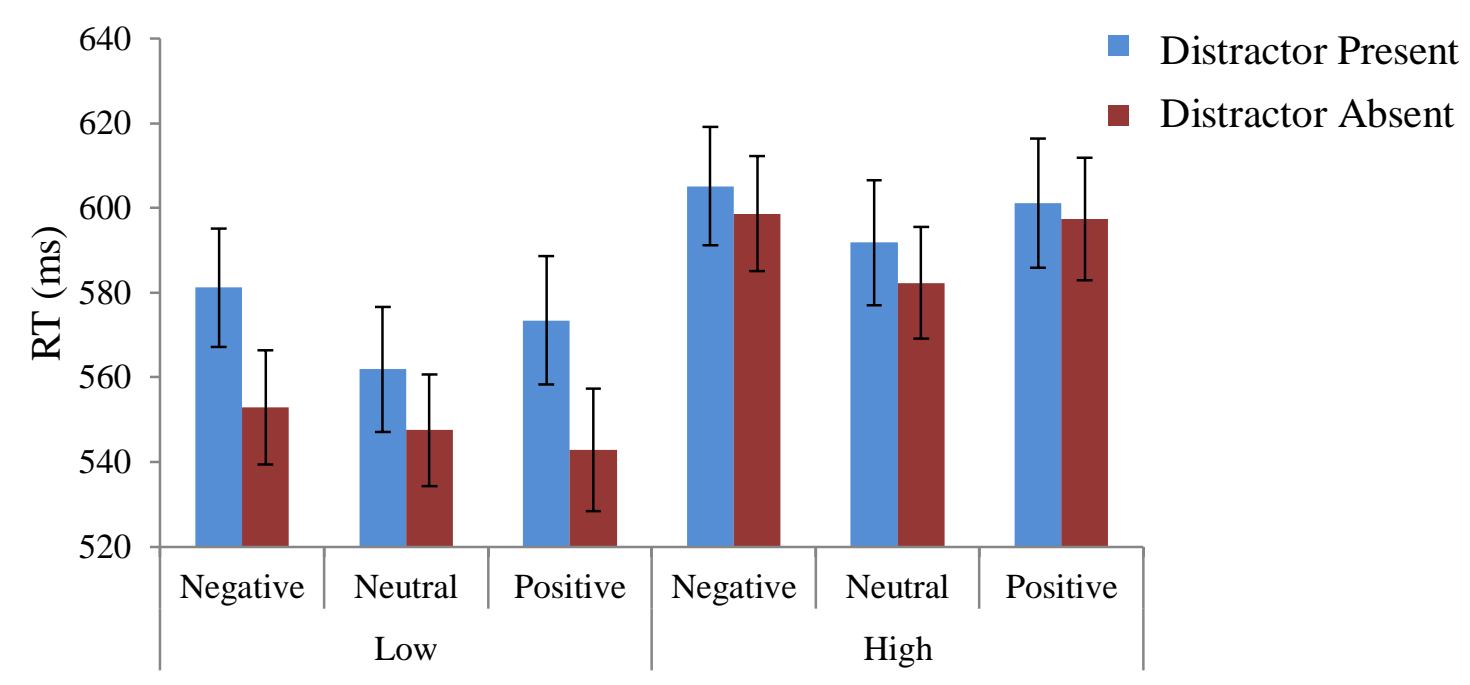

Distractor Frequency

Figure 10. Mean RTs on the distraction task, for participants who met the behavioural inclusion criteria, by distractor frequency, valence and distractor presence in Experiment 2. Note. Bars represent standard errors of the mean

To follow-up the previous analysis, and further examine hypothesised differences in distraction depending on the valence of the image, distraction indices were calculated (RT on distractor-present - RT on distractor-absent trials). For the following analyses quadratic relationships for valence $\mathrm{x}$ distractor frequency were examined as this interaction captures the predicted difference in emotional distraction between the high and low distractor frequency conditions. A 2 (distractor frequency: low, high) x 3 (valence: negative, neutral, positive) mixed ANOVA was conducted. See Table 8 for mean distraction indices and standard deviations by distractor frequency and valence. This analysis yielded a significant main effect of distractor frequency, $F(1,66)=15.49, p<.001, \eta_{p}{ }^{2}=.19$. Less distraction was observed under high than low distractor frequency conditions, consistent with a shift towards proactive control under high distractor frequency conditions. There was also a significant interaction between valence and distractor frequency, $F(1.66,109.62)=3.63, p=.038, \eta_{p}^{2}=.05$, and a significant valence $\mathrm{x}$ distractor frequency quadratic interaction, $F(1,66)=5.56, p=.021, \eta_{p}{ }^{2}$ $=.08$. 
To follow-up these interactions a one-way repeated measures ANOVA, with valence (negative, neutral, positive) as the within subject variable and distraction index as the dependent variable, was conducted for the low and high distractor frequency conditions separately. In the low distractor frequency condition, there was a significant effect of valence, $F(2,66)=4.40, p=.016, \eta_{p}^{2}=.12$, and a significant quadratic relationship for valence, $F(1$, $33)=6.87, p=.013, \eta_{p}^{2}=.17$. As shown in Figure 11, the results largely replicated those in Experiment 1, showing more distraction from emotional than neutral images. Follow-up ttests showed significantly more distraction from positive than from neutral, images, $t(33)$ $=2.95, p=.006, d=.52$ and marginally more distraction from negative than from neutral images, $t(33)=1.99, p=.055, d=.47$. Distraction from positive and negative images did not differ, $t(33)=.46, p=.652$.

In the high distractor frequency condition, no effect of valence, and no quadratic relationship for valence, was observed. In conjunction with the analysis above, this suggests that the very small $(7 \mathrm{~ms})$, but significant, distraction under high distractor frequency conditions was not modulated by valence of the distractor - i.e., proactive control was equally as effective for all distractor types. Taken together, the results replicate Experiment 1 and show that emotional distractors are only more potent than neutral distractors under conditions that evoke reactive cognitive control. When proactive control is engaged, emotional distractions are controlled as effectively as neutral distractions. 


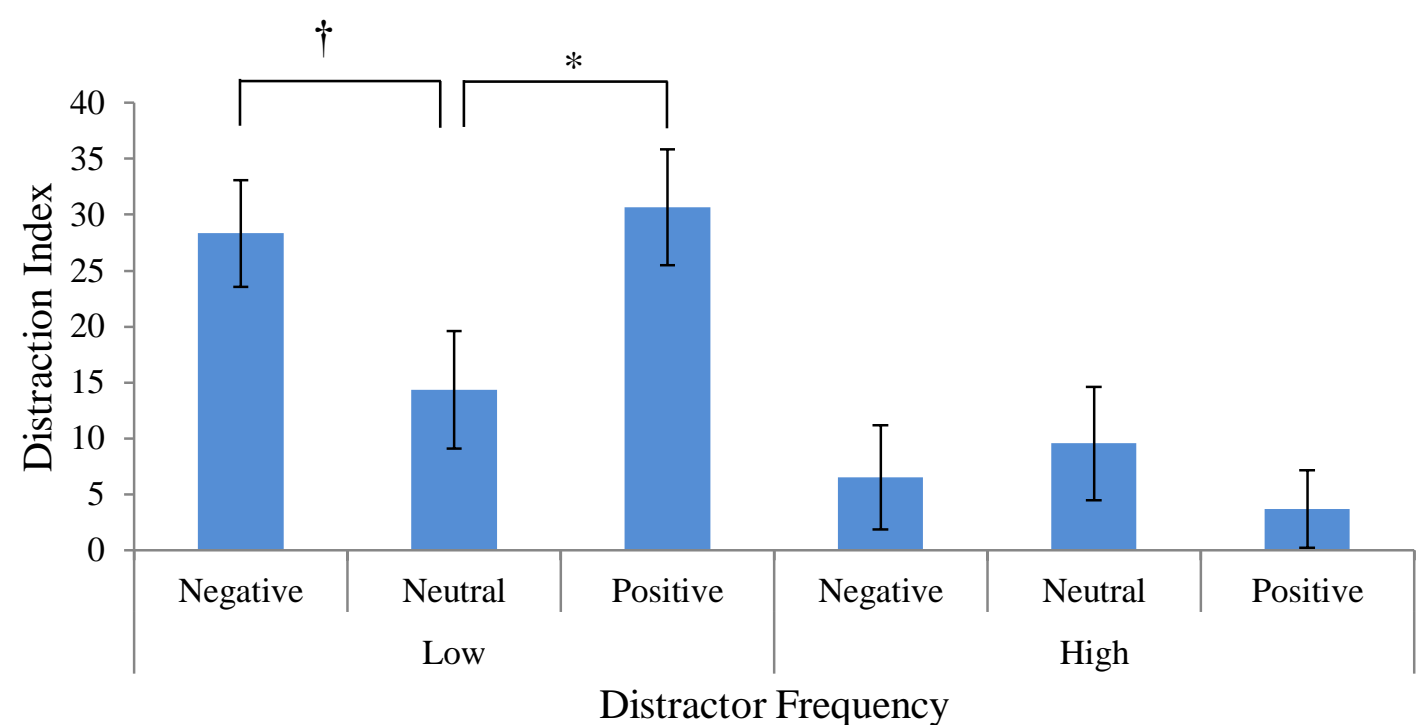

Figure 11. Mean distraction Index (RT on distractor-present trials - RT on distractor-absent trials), for participants who met the behavioural inclusion criteria, by distractor frequency and valence in Experiment 2. Pronounced emotional (compared to neutral) distraction was observed in the low distractor frequency condition. No difference between emotional and neutral distraction was observed in the high distractor frequency condition.

Note. Bars represent standard errors of the mean

Note. $*=p<.05, \uparrow=p<.1$

Follow-up Analysis on the Main Effect of RT. One unexpected result was that RTs were slower overall under high, than low, distractor frequency conditions. One possible explanation for this slowing in RT is that sustained proactive control might have led to a gradual increase in RT across the experiment (i.e., the longer it was maintained). If this were the case, then RTs should be similar between the high and low distractor frequency condition at the beginning of the experiment. An independent samples t-test on RTs for practice trials $(\mathrm{N}=24)$ showed that responses were marginally slower in the high distractor frequency condition $(M=675, S D=11)$, than the low distractor frequency condition $(M=629, S D=$ $94), t(66)=1.82, p=.074, d=.44$. This suggests that the RT difference was due to a difference between groups that was present from the beginning of the experiment, rather than one that developed across time in response to task conditions.

\section{Accuracy.}

Overall, accuracy rates were very high (see Figure 12). Accuracy scores (\%) were analysed in a 2 (distractor frequency: low, high) x 3 (valence: negative, neutral, positive) x 2 (distractor presence: present, absent) mixed ANOVA. For mean accuracy scores and standard deviations by distractor frequency, valence and distractor presence see Table 9 . This analysis 
yielded a significant valence $\mathrm{x}$ distractor frequency interaction, $F(2,132)=3.11, p=.048$, $\eta_{p}{ }^{2}=.05$. No other main effects or interactions were significant. To follow-up the valence by distractor frequency interaction, data were split by distractor frequency. In the low distractor frequency condition, a main effect of valence was observed, $F(2,66)=3.75, p=.029, \eta_{p}{ }^{2}=$ .10. As shown in Figure 12, accuracy was highest in positive blocks of trials, lowest in negative blocks of trials, and intermediate in neutral blocks of trials (although differences were slight, as accuracy was high across all conditions). Follow-up tests showed that accuracy was significantly lower in negative blocks of trials, than positive blocks of trials, $t(33)=2.79, p=.009, d=.38$. However accuracy did not significantly differ between neutral and negative, $t(33)=1.16, p=.256$, or neutral and positive, $t(33)=1.53, p=.135$, blocks. In the high distractor frequency condition, no effect of valence was observed. Together, these findings suggest that when reactive control is engaged, the presence of negative distractors in a block of trials impairs overall accuracy. This decrease in accuracy could be the byproduct of a mood effect from constantly processing negative images. Importantly, the pattern of accuracy data does not suggest that the increase in emotional distraction (as measured by RTs) under low distractor frequency conditions was due to a speed-accuracy trade off.

Table 9

Mean (SD) accuracy (\%) for the distraction task, for participants who met the behavioural inclusion criteria, by distractor frequency, valence and distractor presence in Experiment 2.

\begin{tabular}{lcccc}
\hline & \multicolumn{2}{c}{ Low Distractor Frequency } & \multicolumn{2}{c}{ High Distractor Frequency } \\
& Present & Absent & Present & Absent \\
Valence & M (SD) & M (SD) & M (SD) & M (SD) \\
\hline Negative & $94.70(4.50)$ & $95.57(2.87)$ & $96.00(3.04)$ & $95.88(3.49)$ \\
Neutral & $95.34(2.95)$ & $95.85(3.05)$ & $95.74(3.35)$ & $95.34(3.84)$ \\
Positive & $96.51(3.02)$ & $95.93(2.33)$ & $95.39(3.46)$ & $95.78(3.21)$
\end{tabular}




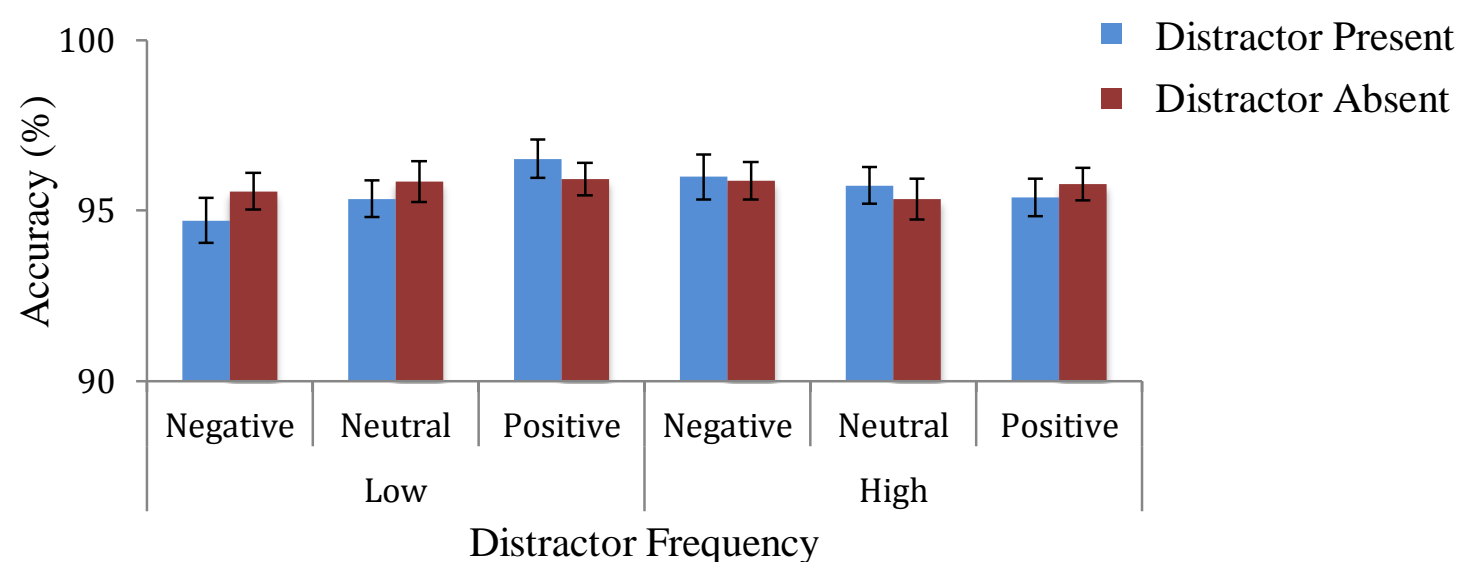

Figure 12. Mean accuracy (\%) on the distraction task, for participants who met the behavioural inclusion criteria, by distractor frequency, valence and distractor presence in Experiment 2.

Note. Bars represent standard errors of the mean

\section{Follow-up Behavioural Analyses}

I analysed all participants' data first to determine whether the pattern of results was similar to Experiment 1. However, as some participants' data were removed for ERP analyses due to artifact rejection (but kept in the behavioural analyses above), behavioural data were similarly analysed using data from only the subset of people who were kept in the ERP analyses (high distractor frequency: $\mathrm{N}=31$; low distractor frequency: $\mathrm{N}=32$ ). Behavioural data from the subset of ERP participants was analysed to (1) see if they showed the same pattern of results, and (2) allow for more direct comparisons of behavioural and ERP effects.

\section{Response Times.}

RTs were analysed in a mixed 2 (distractor frequency: low, high) x 3 (valence: negative, neutral, positive) x 2 (distractor presence: present, absent) ANOVA. For mean RTs and standard deviations by distractor frequency, valence and distractor presence see Table 10. Overall, the pattern of results was similar to above (see Figure 13). Significant distraction was observed; RTs were slower on distractor-present, relative to distractor-absent trials, $F(1$, $61)=57.50, p<.001, \eta_{p}{ }^{2}=.49$. Further, responses were slower in the high distractor frequency than in the low distractor frequency condition, $F(1,61)=4.32, p=.042, \eta_{p}^{2}=.07$. A significant two way distractor presence $\mathrm{x}$ distractor frequency interaction was observed, $F(1,61)=20.33, p<.001, \eta_{p}{ }^{2}=.24$. Consistent with the assumption that high distractor frequency evokes proactive control strategies, there was less distraction in the high (6ms of distraction), $t(30)=2.04, p=.05, d=.06$, than the low (22ms of distraction), $t(31)=9.14, p$ $<.001, d=.35$, distractor frequency condition. Importantly, while the pattern of results for 
this subset of participants were similar to the pattern of results for all participants (see Figure 13), the critical three way valence $\mathrm{x}$ distractor presence $\mathrm{x}$ distractor frequency interaction was not significant, $F(1.72,104.83)=2.40, p=.104, \eta_{p}^{2}=.04$.

Table 10

Mean (SD) RTs (ms) for the distraction task, for participants who met the behavioural inclusion criteria, by distractor frequency, valence and distractor presence in Experiment 2.

\begin{tabular}{ccccccc}
\hline & \multicolumn{3}{c}{ Low Distractor Frequency } & \multicolumn{3}{c}{ High Distractor Frequency } \\
& Distractor & Distractor & Distraction & Distractor & Distractor & Distraction \\
& Present & Absent & Index & Present & Absent & Index \\
Valence & M (SD) & M (SD) & M (SD) & M (SD) & M (SD) & M (SD) \\
\hline Negative & $572(66)$ & $549(61)$ & $23(19)$ & $602(85)$ & $596(93)$ & $6(27)$ \\
Neutral & $559(72)$ & $544(72)$ & $15(28)$ & $59096)$ & $581(83)$ & $9(29)$ \\
Positive & $567(72)$ & $539(66)$ & $28(20)$ & $601(99)$ & $599(103)$ & $2(20)$ \\
\hline
\end{tabular}

Note. Distraction Index = RT on distractor-present trials - RT on distractor-absent trials.

Even though the predicted three-way interaction did not reach significance $(p=.104)$, it was still followed up for completeness, and to aid interpretation of the ERP results. A 3 (valence: negative, neutral, positive) x 2 (distractor presence: present, absent) repeated measures ANOVA was conducted separately for the low and high distractor frequency conditions. In the low distractor frequency condition, a main effect of distractor presence, $F(1,31)=83.50, p<.001, \eta_{p}{ }^{2}=.729$, indicated that there was significant distraction. Importantly, a marginal two-way valence x distractor presence interaction was observed, $F(2$, $62)=2.75, p=.072, \eta_{p}{ }^{2}=.08$. As depicted in Figure 13, the pattern of data was consistent with an emotional distraction effect; distraction was largest from positive images ( $28 \mathrm{~ms}$ of distraction), $t(31)=7.91, p<.001, d=.41$, slightly smaller from negative images ( $23 \mathrm{~ms}$ of distraction), $t(31)=7.00, p<.001, d=.36$, and smallest from neutral images ( $15 \mathrm{~ms}$ of distraction), $t(31)=2.99, p=.005, d=.21$.

In the high distractor frequency condition, a main effect of distractor presence indicated that significant distraction was observed, $F(1,30)=4.17, p=.050, \eta_{p}^{2}=.12$. No other interactions or main effects were significant. As expected, this suggests that when proactive control is engaged emotional distractions are as effectively controlled as neutral distractions. 


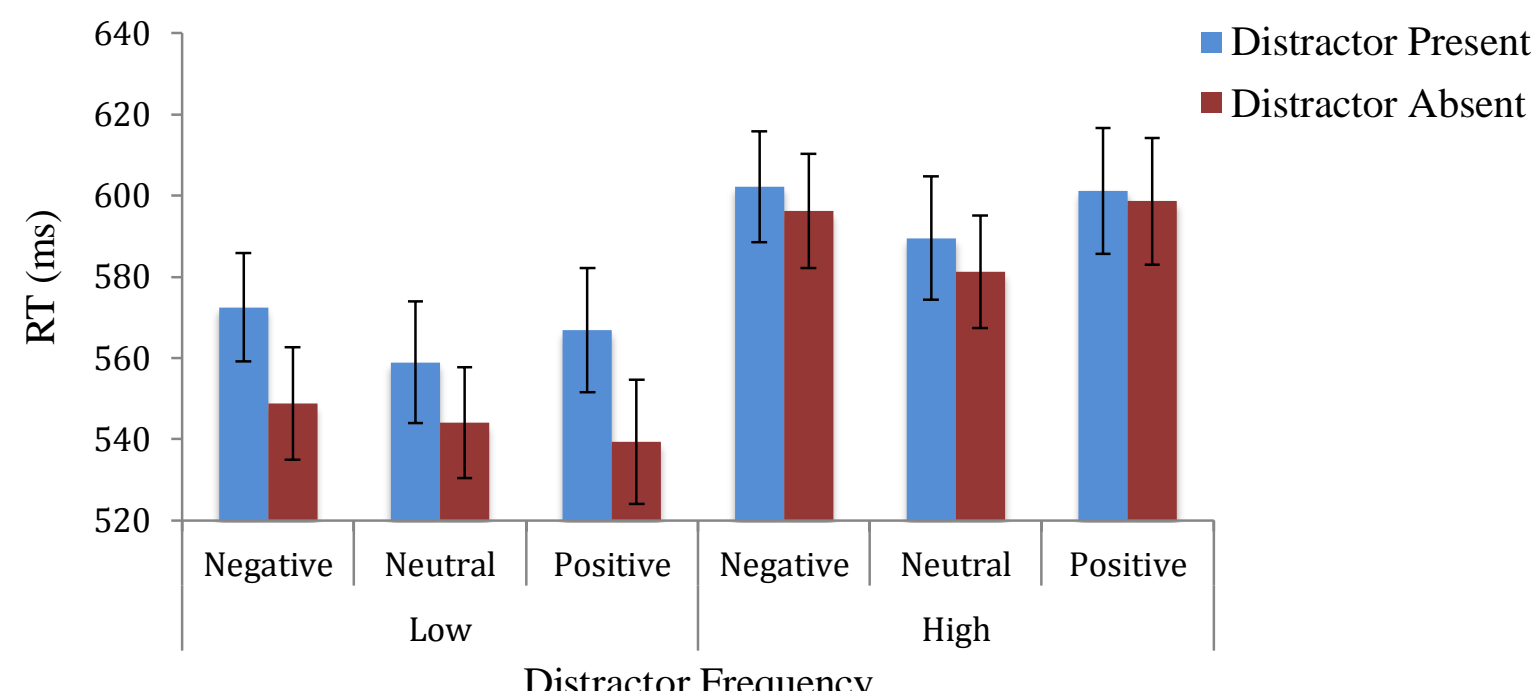

Figure 13. Mean RTs on the distraction task, for participants who met the ERP inclusion criteria, by distractor frequency, valence and distractor presence in Experiment 2. Note. Bars represent standard errors of the mean

As above, data were followed up by examining distraction indices (RTs on distractorpresent trials - RTs on distractor-absent trials). For mean distraction indices and standard deviations by distractor frequency, and valence see Table 10. A 2 (distractor frequency: low, high) x 3 (valence: negative, positive, neutral) mixed ANOVA was conducted. This analysis yielded a non-significant interaction between valence and distractor frequency, $F(1.72$, $104.83)=2.40, p=.104, \eta_{p}^{2}=.04$, but a marginally significant (and predicted) valence $\mathrm{x}$ distractor frequency interaction for quadratic relationships, $F(1,61)=3.32, p=.073, \eta_{p}{ }^{2}=$ .05 .

To follow-up this marginal interaction a one-way repeated measures ANOVA, with valence (negative, neutral, positive) as the within subject variable was conducted for the low and high distractor frequency conditions separately. In the low distractor frequency condition there was a marginal effect of valence, $F(2,62)=2.75, p=.072, \eta_{p}{ }^{2}=.08$, and a significant quadratic effect for valence, $F(1,31)=4.25, p=.048, \eta_{p}^{2}=.12$. As shown in Figure 14, the pattern of data was consistent with an emotional distraction effect; distraction was greatest from positive images, least from neutral images, and intermediate from negative images. Follow-up tests showed significantly more distraction from positive than from neutral images, $t(31)=2.47, p=.019, d=.53$. Distraction from negative images did not differ significantly from positive images, $t(31)=.740, p=.465$, or neutral images, $t(31)=1.397, p$ $=.172$. This pattern of data shows the expected positive emotional distraction effect; when 
reactive control is engaged, positive images are more distracting than neutral images.

However, inconsistent with predictions, these findings do not indicate that a reliable negative emotional distraction effect was observed.

Under high distractor frequency conditions there was no effect of valence (see Figure 14). This suggests proactive mechanisms controlled emotional distraction as effectively controlled as neutral distraction. Taken together, the pattern of data is fairly consistent with predictions and suggests that emotional distraction (particularly for positive images) emerges under reactive (but not proactive) control conditions. However, the interaction between valence and distractor frequency is marginal, thus results should be interpreted in this manner with caution particularly as the three way distractor presence $\mathrm{x}$ valence $\mathrm{x}$ distractor frequency interaction reported in the analysis above was not significant $(p=.104)$ in the subset of people whose data was included in the subsequent ERP analyses.

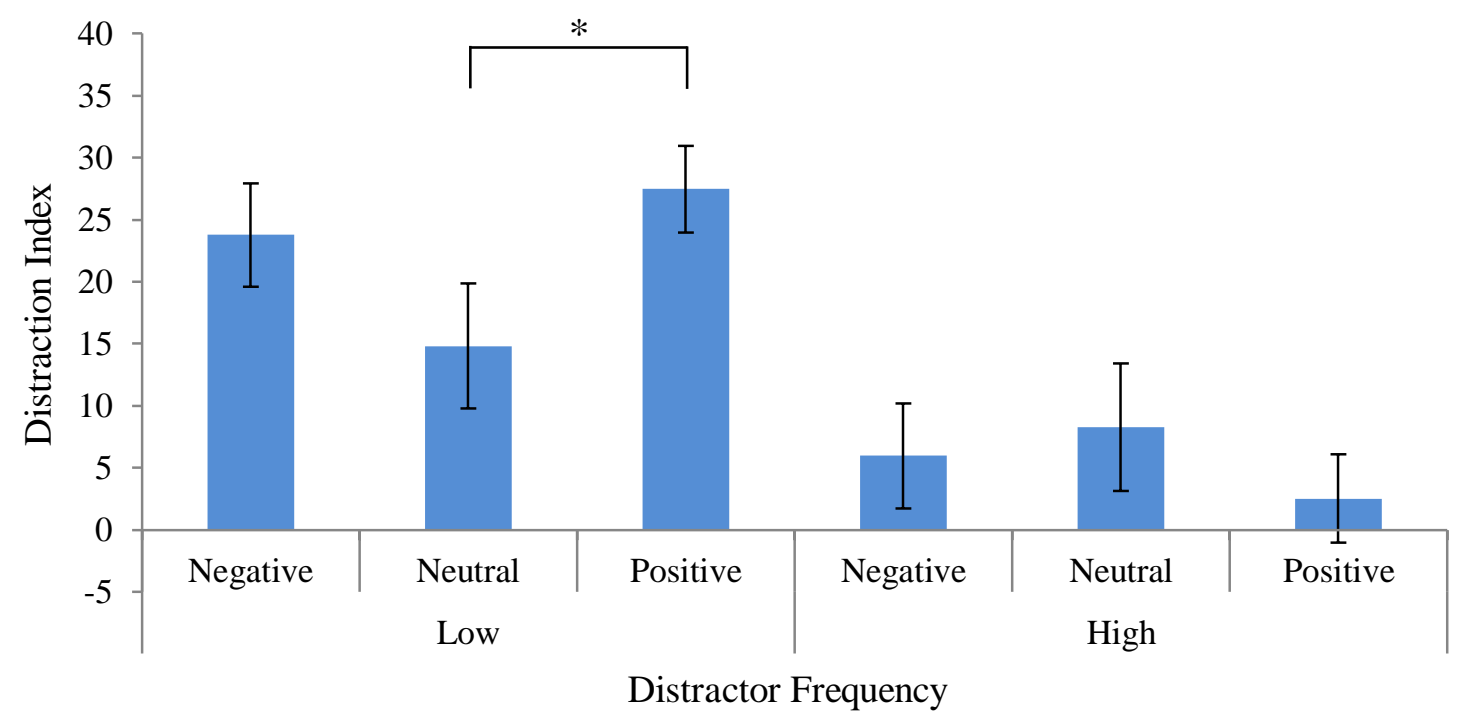

Figure 14. Mean distraction Index (RT on distractor-present trials - RT on distractor-absent trials), for participants who met the ERP inclusion criteria, by distractor frequency and valence in Experiment 2. Pronounced positive, but not negative, (compared to neutral) distraction was observed in the low distractor frequency condition. No difference between emotional and neutral distraction was observed in the high distractor frequency condition.

Note. Bars represent standard errors of the mean

Note. $*=p<.5$

\section{Accuracy.}

Accuracy was again very high in the subset of participants included in ERP analyses. Accuracy scores (\%) were analysed in a 2 (distractor frequency: low, high) x 3 (valence: 
negative, neutral, positive) x 2 (distractor presence: present, absent) mixed ANOVA. For mean accuracy (\%) and standard deviations by distractor frequency, valence and distractor presence see Table 11. This analysis yielded a marginal valence by distractor frequency interaction, $F(2,122)=2.70, p=.071, \eta_{p}^{2}=.04$, that was qualified by a significant valence $\mathrm{x}$ distractor presence $\mathrm{x}$ distractor frequency interaction, $F(2,122)=3.40, p=.037, \eta_{p}{ }^{2}=.05$. This interaction was followed up by conducting a 3 (valence: negative, neutral, positive) x 2 (distractor presence: present, absent) repeated measures ANOVA for the low and high distractor frequency conditions separately. In the low distractor frequency condition, a marginal main effect of valence, $F(2,62)=3.04, p=.055, \eta_{p}{ }^{2}=.09$, was observed that was qualified by a marginal valence $\mathrm{x}$ distractor presence interaction, $F(1.69,52.27)=2.60, p=$ $.082, \eta_{p}{ }^{2}=.08$. Follow-up tests showed that there was no significant difference between accuracy for distractor-present and absent trials in any condition (all t's $<1.58$ ) - i.e., no distraction effects. However, as depicted in Figure 15, neutral and negative images showed the expected pattern of distraction - accuracy was lower on distractor-present, than distractorabsent trials. Positive images instead showed a reversed distraction effect (that is, accuracy was higher when distractors were present versus absent). In the high distractor frequency condition, no main effects or interactions were significant. Taken together, although findings for positive distractors might suggest some speed-accuracy trade-off, it is important to bear in mind that none of the distraction effects were themselves significant, and accuracy overall was very high. Thus, even if there is a slight trade-off in speed for accuracy for positive distractor trials that cannot account for the pattern of pronounced positive distraction seen in the RT data.

\section{Table 11}

Mean (SD) accuracy (\%) for the distraction task, for participants who met the behavioural inclusion criteria, by distractor frequency, valence and distractor presence in Experiment 2.

\begin{tabular}{lcccc}
\hline & \multicolumn{2}{c}{ Low Distractor Frequency } & \multicolumn{2}{c}{ High Distractor Frequency } \\
& Distractor & Distractor & Distractor & Distractor \\
& Present & Absent & Present & Absent \\
Valence & M (SD) & M (SD) & M (SD) & M (SD) \\
\hline Negative & $94.64(4.63)$ & $95.61(2.94)$ & $95.93(3.18)$ & $95.81(3.57)$ \\
Neutral & $95.47(2.97)$ & $95.82(3.12)$ & $95.57(3.46)$ & $95.11(3.94)$ \\
Positive & $96.46(3.10)$ & $95.83(2.32)$ & $95.14(3.49)$ & $95.91(3.27)$ \\
\hline
\end{tabular}




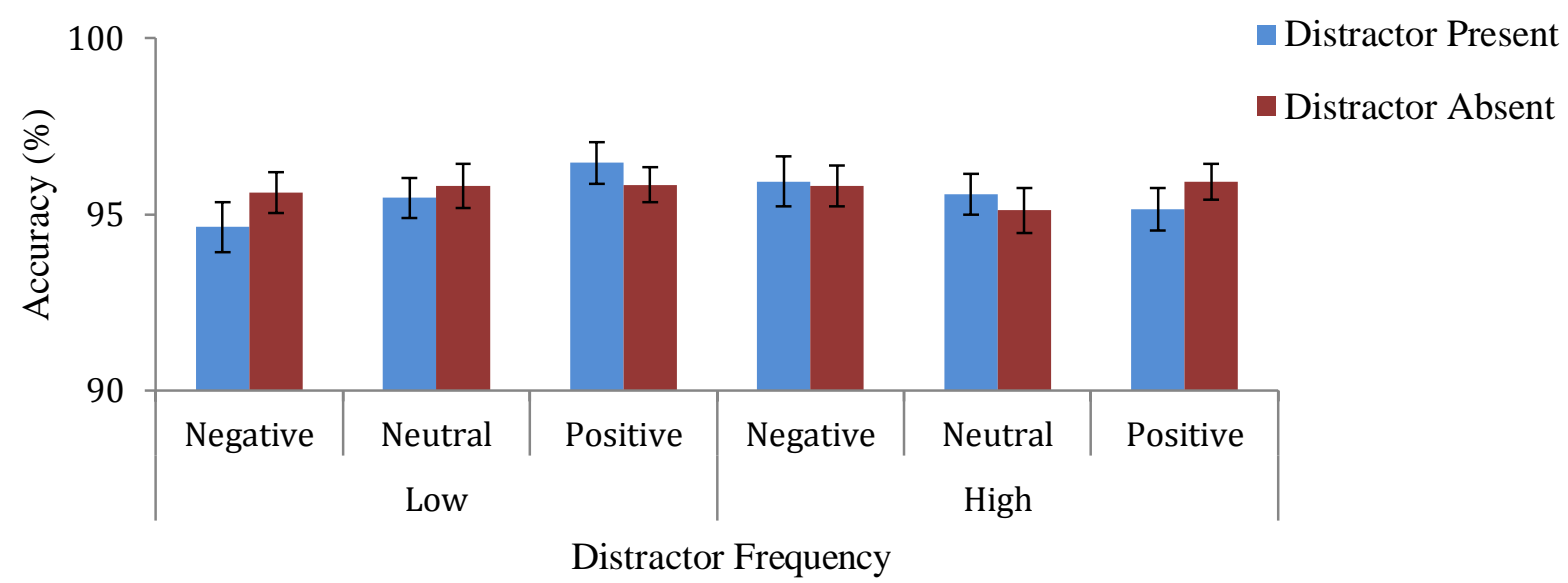

Figure 15. Mean accuracy (\%) on the distraction task, for participants who met the ERP inclusion criteria, by distractor frequency, valence and distractor presence in Experiment 2. Note. Bars represent standard errors of the mean

\section{ERP Results}

For all ERP analyses only distractor-present trials were analysed as an index of emotional processing. As in the behavioural results above, quadratic effects were examined for predicted relationships (i.e., valence and valence x distractor frequency effects). Given the exploratory nature of the ERP study, marginal effects were followed up but only if they were predicted or addressed the primary hypotheses.

\section{LPP (400ms to $800 \mathrm{~ms})$.}

The LPP waveforms for negative, neutral and positive images are presented in Figure 16 for the low distractor frequency condition, and Figure 17 for the high distractor frequency condition. For mean LPP amplitudes and standard deviations, collapsed across electrode, by distractor frequency and valence see Table 12. LPP amplitudes were analysed in a 2 (distractor frequency: high, low) x 3 (valence: negative, neutral, positive) x 5 (electrode: PZ, P3, P4, CP3, CP4) mixed ANOVA. A significant main effect of electrode was observed, $F(2.30,140.19)=23.91, p<.001, \eta_{p}{ }^{2}=.28$, indicating that mean activity was greater at $\mathrm{PZ}$ and $\mathrm{CP} 4$ than $\mathrm{CP} 3, \mathrm{P} 3$ and P4. However, as electrode did not interact with any other variables of interest, electrode site was not considered any further. There was also a main effect of valence, $F(2,122)=14.34, p<.001, \eta_{p}{ }^{2}=.19$, and a significant quadratic effect of valence, $F(1,61)=22.52, p<.001, \eta_{p}{ }^{2}=.27$. Consistent with typical emotional modulation of the LPP, follow-up tests confirmed that amplitudes were larger for positive than neutral images, $t(62)=5.08, p<.001, d=.50$, and for negative than neutral images, $t(62)=2.74, p=.008, \mathrm{~d}$ $=.25$. However, unexpectedly, the LPP was also larger for positive images than negative 
images, $t(62)=2.76, p=.008, d=.27$. The predicted interaction between valence and distractor frequency was not observed, $F(2,122)=.178, p=.178, \eta_{p}^{2}=.03$, however the predicted valence $\mathrm{x}$ distractor frequency quadratic interaction was marginally significant, $F(1,61)=3.80, p=.056, \eta_{p}^{2}=.06$. Data were collapsed across electrodes for the following analyses.

Table 12

Mean (SD) LPP amplitudes ( $\mu V)$, collapsed across electrode, by distractor frequency and valence.

\begin{tabular}{lcc}
\hline & $\begin{array}{c}\text { Low Distractor } \\
\text { Frequency }\end{array}$ & $\begin{array}{c}\text { High Distractor } \\
\text { Frequency }\end{array}$ \\
Valence & $\mathrm{M}(\mathrm{SD})$ & $\mathrm{M}(\mathrm{SD})$ \\
\hline Negative & $4.02(3.38)$ & $2.93(2.77)$ \\
Neutral & $2.71(3.65)$ & $2.70(2.74)$ \\
Positive & $4.84(3.93)$ & $3.90(2.78)$ \\
\hline
\end{tabular}

To further explore the marginal (but predicted) valence $\mathrm{x}$ distractor frequency quadratic interaction, repeated measures one-way ANOVAs with valence (negative, neutral, positive) as the within subjects variable were conducted on LPP amplitudes for the high and low distractor frequency conditions separately. In the low distractor frequency condition, there was a main effect of valence, $F(2,62)=9.24, p<.001, \eta_{p}^{2}=.230$, and a significant quadratic relationship for valence, $F(1,31)=17.21, p<.001, \eta_{p}{ }^{2}=.36$. As shown in Figure 16, LPP amplitudes were largest for positive images, smallest for neutral images, and intermediate for negative images. Follow-up tests indicated that the LPP was significantly potentiated for positive images compared to neutral images, $t(31)=2.04, p=.005, d=.56$, and for negative images compared to neutral images, $t(31)=3.93, p<.001, d=.37$. As shown in Figures 18B and 19B the emotional-neutral differences in LPP amplitudes had fairly typical centro-parietal scalp distributions. There was no significant difference between the magnitude of the LPP elicited by positive and negative images, $t(31)=1.58, p=.125$. These findings are in line with predictions and suggest that when reactive control processes are relied on, emotional distractors enhance the LPP - i.e., attention is sustained to emotional distractors to a greater extent than to neutral distractors.

In the high distractor frequency condition, there was a significant main effect of valence, $F(2,60)=6.06, p=.004, \eta_{p}{ }^{2}=.17$, and a significant quadratic effect for valence, 
$F(1,30)=6.28, p=.023, \eta_{p}{ }^{2}=.16$. As shown in Figure 17 , the LPP was only modulated by positive images. Follow-up tests indicated that the LPP was larger for positive images compared to neutral images, $t(30)=3.35, p=.002, d=.43$ and negative images, $t(30)=2.51$, $p=.018, d=.35$. However, there was no significant difference between magnitude of the LPP elicited by neutral and negative images, $t(20)=.70, p=.508$. As shown in Figure 18C the positive-neutral difference in LPP amplitudes had fairly typical centro-parietal scalp distribution. The lack of emotional modulation of the LPP by negative images suggests that proactive control reduces sustained attention to emotional characteristics of negative distractors. However, results suggest (unexpectedly) that attention is sustained to positive images even when proactive control is engaged.

As the LPP was potentiated for positive (as compared to neutral) images in both the low and high distractor frequency conditions, a follow-up analysis was conducted to test whether the magnitude of the positive LPP differed between the two distractor frequency conditions. If proactive control reduces sustained attention to positive distractors, then the modulation of the LPP by positive images should be attenuated under high distractor frequency conditions. LPP amplitudes were entered into a 2 (valence: positive, neutral) x 2 (distractor frequency: low, high) mixed ANOVA. The valence by distractor frequency interaction was not significant, $F(1,61)=2.01, p=.161$, suggesting that modulation of the LPP by positive images was similar across distractor frequency conditions. This finding was unexpected, and inconsistent with the hypothesis that proactive control reduces sustained attention to positive images. 


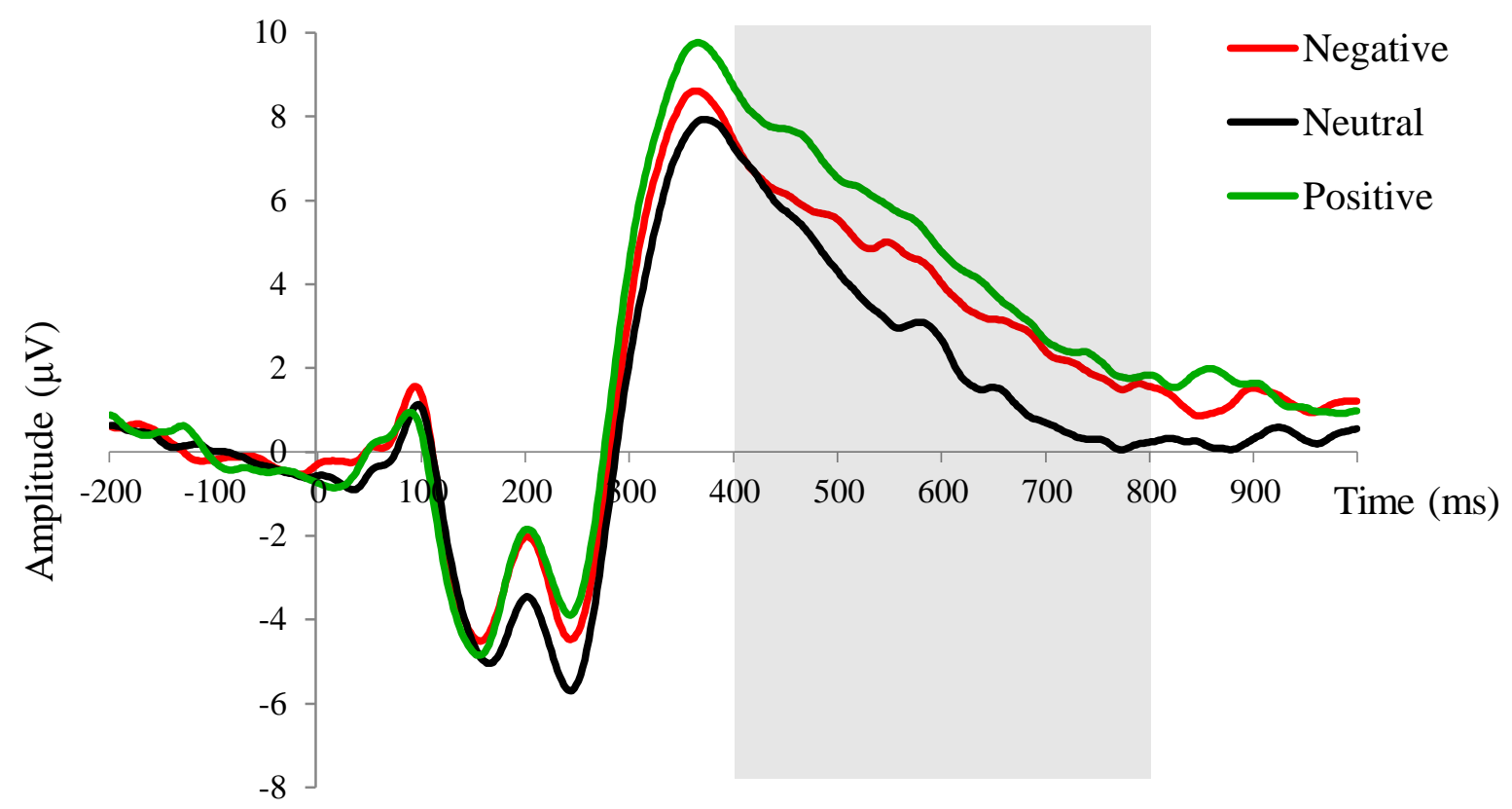

Figure 16. LPP amplitudes in the low distractor frequency condition collapsed across electrodes (PZ, CP3, CP4, P3, P4) by valence. Positive and negative images elicited a larger (i.e., more positive) LPP than neutral images. There was no difference in LPP amplitudes elicited by positive and negative images.

Note. Shaded area represents timeframe for the LPP (400ms to $800 \mathrm{~ms}$ )

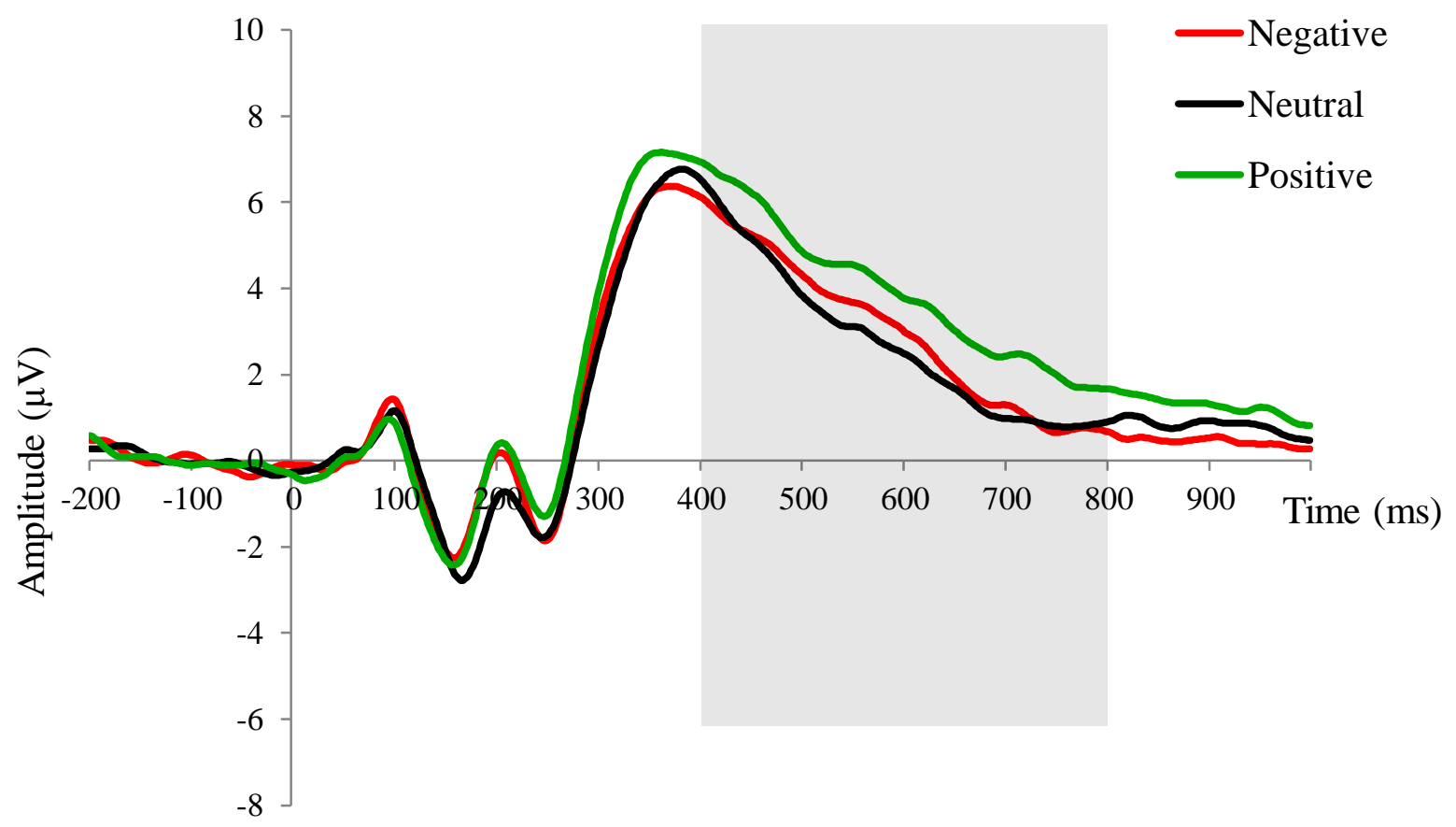

Figure 17. LPP amplitudes in the high distractor frequency condition by valence. Positive images elicited a larger (i.e., more positive) LPP than neutral and negative images. There was no difference in LPP amplitudes elicited by neutral and negative images.

Note. Figure represents mean amplitudes collapsed across electrodes (PZ, CP3, CP4, P3, P4) Note. Shaded area represents timeframe for the LPP (400ms to $800 \mathrm{~ms})$ 


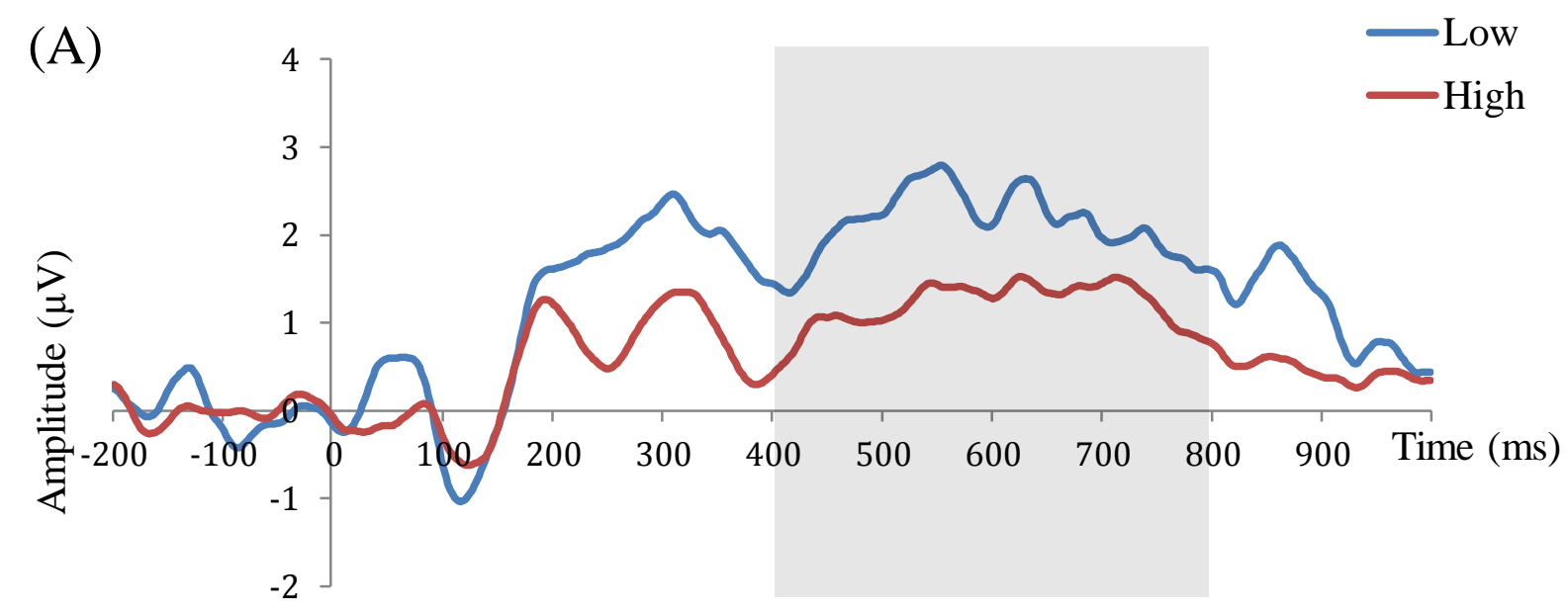

(B) Low
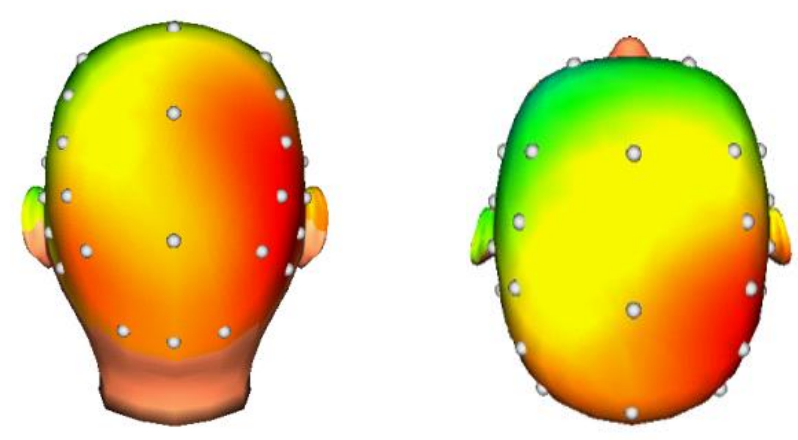

$-2.49$

0

2.49

(C) High
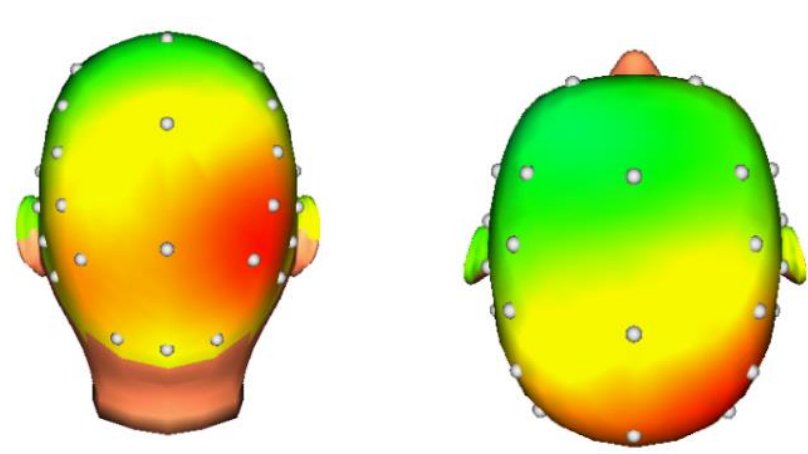

$-1.47$

0

1.47

Figure 18. (A) Difference waves (positive - neutral) for LPPs elicited by positive and neutral images, collapsed across electrodes (PZ, CP3, CP4, P3, P4), by distractor frequency condition. More positive values reflect a larger LPP for positive, compared to neutral, images. Scalp distribution for the positive-neutral difference are depicted for the low distractor frequency condition (B) and high distractor frequency condition (C).

Note. Shaded area in (A) represents timeframe for the LPP (400ms to $800 \mathrm{~ms}$ )

Note. Head maps between distractor frequency conditions are presented on different scales. Scales were chosen to allow detailed representation of the positive-neutral difference 


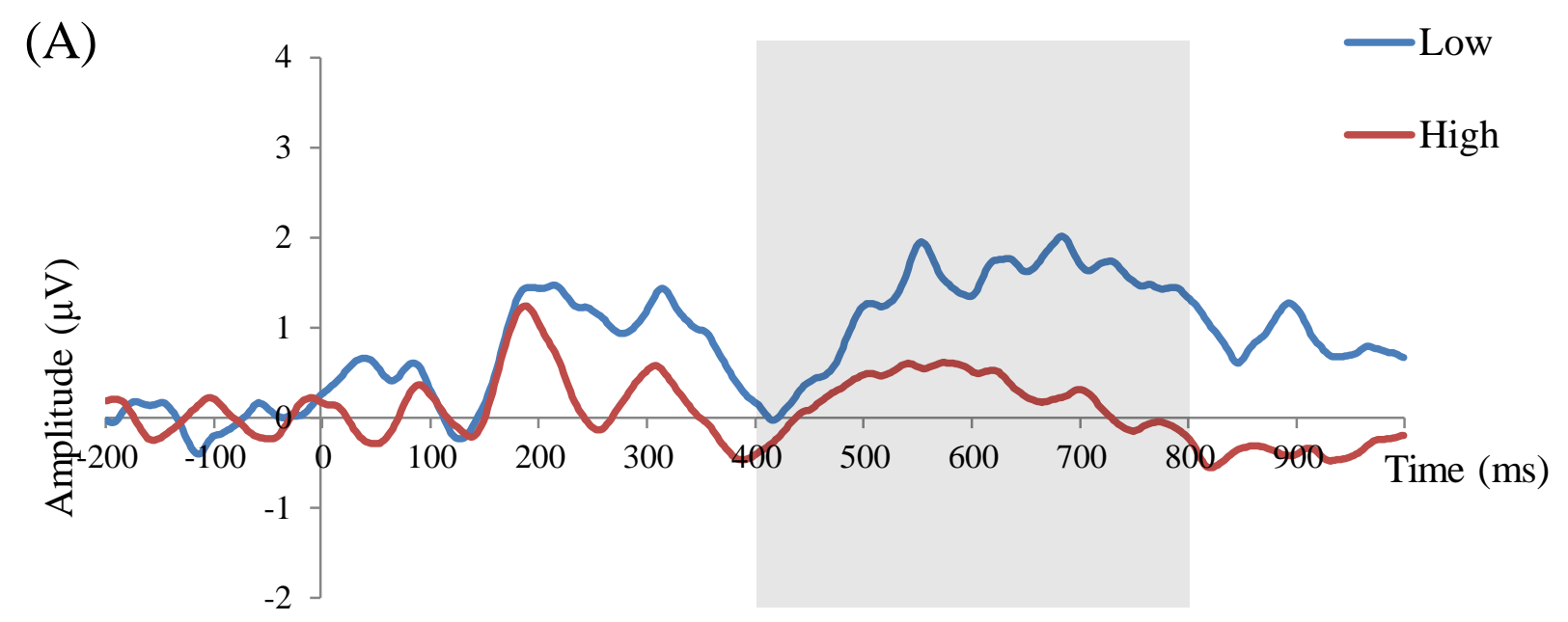

(B) Low
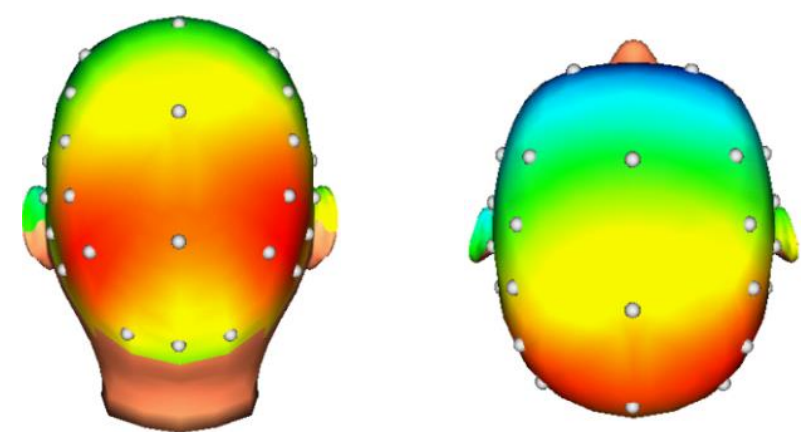

(C) High

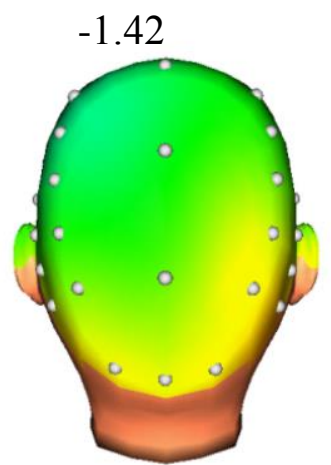

0

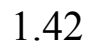

$-1.00$

0

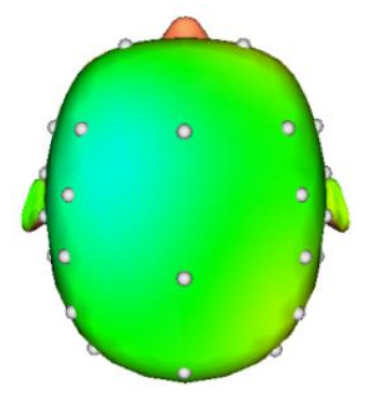

1.00

Figure 19. (A) Difference waves (negative - neutral) for LPPs elicited by negative and neutral images, collapsed across electrodes (PZ, CP3, CP4, P3, P4), by distractor frequency condition. More positive values reflect a larger LPP for negative, compared to neutral, images. Scalp distribution for the negative-neutral difference are depicted for the low distractor frequency condition (B) and high distractor frequency condition (C). Note. Shaded area in (A) represents timeframe for the LPP (400ms to $800 \mathrm{~ms}$ ) Note. Head maps between distractor frequency conditions are presented on different scales. Scales were chosen to allow detailed representation of the positive-neutral difference 


\section{EPN (180ms to 260ms).}

The EPN waveforms for negative, neutral and positive images are presented in Figure 20 for the low distractor frequency condition, and Figure 21 for the high distractor frequency condition. For mean EPN amplitudes and standard deviations, collapsed across electrode, by distractor frequency and valence, see Table 13. EPN amplitudes were entered into a 2 (distractor frequency: low, high) x 3 (valence: negative, neutral, positive) x 3 (electrode: O1, OZ, O2) mixed ANOVA. A significant main effect of electrode was observed, $F(1.23,74.82)$ $=4.64, p=.027, \eta_{p}{ }^{2}=.07$, indicating that mean activity was greater at $\mathrm{O} 1$ and $\mathrm{O} 2$ than $\mathrm{OZ}$. Further, a marginal electrode $\mathrm{x}$ valence interaction was observed, $F(4,168.49)=2.18, p=$ $.097, \eta_{p}{ }^{2}=.04$. This marginal interaction was not followed-up because it was not predicted nor relevant to hypotheses. There was a significant main effect of valence, $F(2,122)=6.81, p$ $=.002, \eta_{p}{ }^{2}=.10$, and a significant quadratic effect of valence, $F(1,61)=8.04, p=.006, \eta_{p}{ }^{2}=$ .05. Follow-up tests showed that EPN was more pronounced (i.e., more negative) for positive images than neutral images, $t(62)=3.36, p<.001, d=.18$, and negative images $t(62)=2.46$, $p=.017, d=.14$. However, EPN amplitudes did not differ between neutral and negative images, $t(62)=1.01, p=.315$. This effect is consistent with the typical positive emotional modulation of the EPN, but (unexpectedly) does not show the typical negative emotional modulation of the EPN. The predicted valence $\mathrm{x}$ distractor frequency interaction, $F(2,122)=$ $2.00, p=.140$, was not significant. However a marginally significant (and predicted) valence by distractor frequency quadratic effect was observed, $F(1,61)=3.05, p=.086, \eta_{p}{ }^{2}=.05$.

Data were collapsed across electrodes for the following analyses.

Table 13

Mean (SD) EPN amplitudes ( $\mu V)$, collapsed across electrode, by distractor frequency and valence.

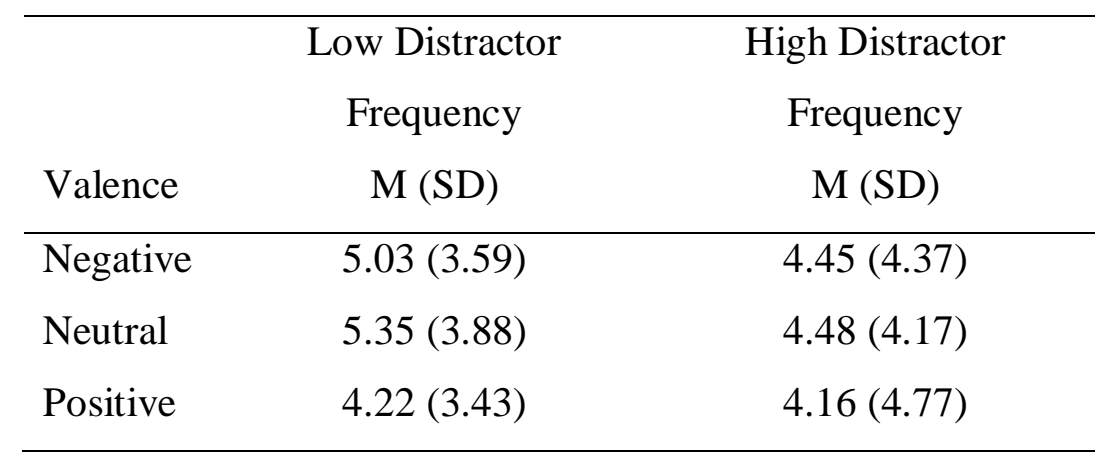

To further explore the marginal (but predicted) valence $\mathrm{x}$ distractor frequency quadratic interaction, repeated measures one-way ANOVAs with valence (negative, neutral, 
positive) as the within subjects variable were conducted on EPN amplitudes for the high and low distractor frequency conditions separately. In the low distractor frequency condition there was a significant main effect of valence, $F(2,62)=6.42, p=.003, \eta_{p}^{2}=.17$, and a significant quadratic relationship for valence, $F(1,31)=4.95, p=.006, \eta_{p}^{2}=.22$. As shown in Figure 20, positive images elicited a pronounced (i.e. more negative) EPN, but negative images did not. Follow-up tests showed that EPN amplitudes for positive images were significantly more negative than neutral images, $t(31)=3.44, p=.002, d=.31$, and negative images $t(31)=2.23, p=.034, d=.23$. However, no difference between negative and neutral images was observed, $t(31)=1.16, p=.256$. As shown in Figure 22B the positive-neutral difference in EPN amplitudes had fairly typical occipital scalp distribution. While the modulation of the EPN by positive images under low distractor frequency conditions was in line with predictions, it was also predicted that negative images would produce a comparable modulation of the EPN. This pattern of data suggests that under reactive control conditions attentional resources are recruited to a greater extent to perceptually process positive, but not negative, images relative to neutral images. The lack of early attentional allocation of resources to negative (over neutral) distractors is particularly curious given that an LPP was observed for negative images. This apparent contradiction is addressed in the general discussion.

There was no effect of valence in the high distractor frequency condition - i.e., the EPN was not modulated by emotionality of distractors (see Figure 21). The lack of negative emotional modulation of the EPN under high distractor frequency cannot be interpreted as an effect of proactive control, as there was no emotional modulation of the EPN in the low distractor frequency condition. However, the absence of an EPN to positive images under high distractor frequency conditions (in conjunction with the presence of an EPN to positive emotional images under low distractor frequency conditions) suggests that proactive control prevents early allocation of attentional resources to perceptual processing of positive distractors. 


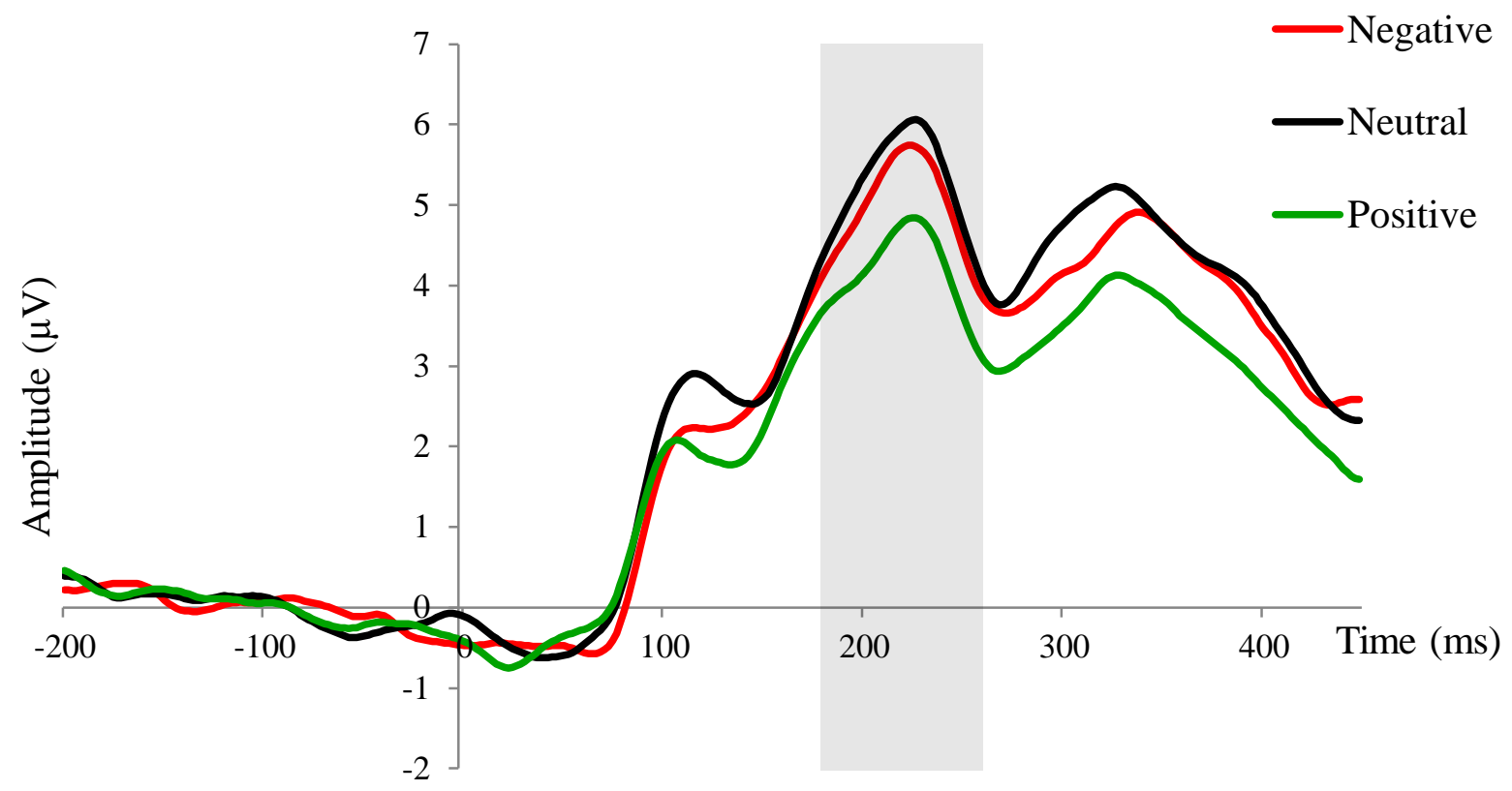

Figure 20. EPN amplitudes in the low distractor frequency condition collapsed across electrodes (O1, OZ, O2), by valence. Positive images elicited a larger (i.e., more negative) EPN than neutral and negative images. There was no difference in EPN amplitudes elicited by neutral and negative images.

Note. Shaded area represents timeframe for the EPN (180ms to $260 \mathrm{~ms})$

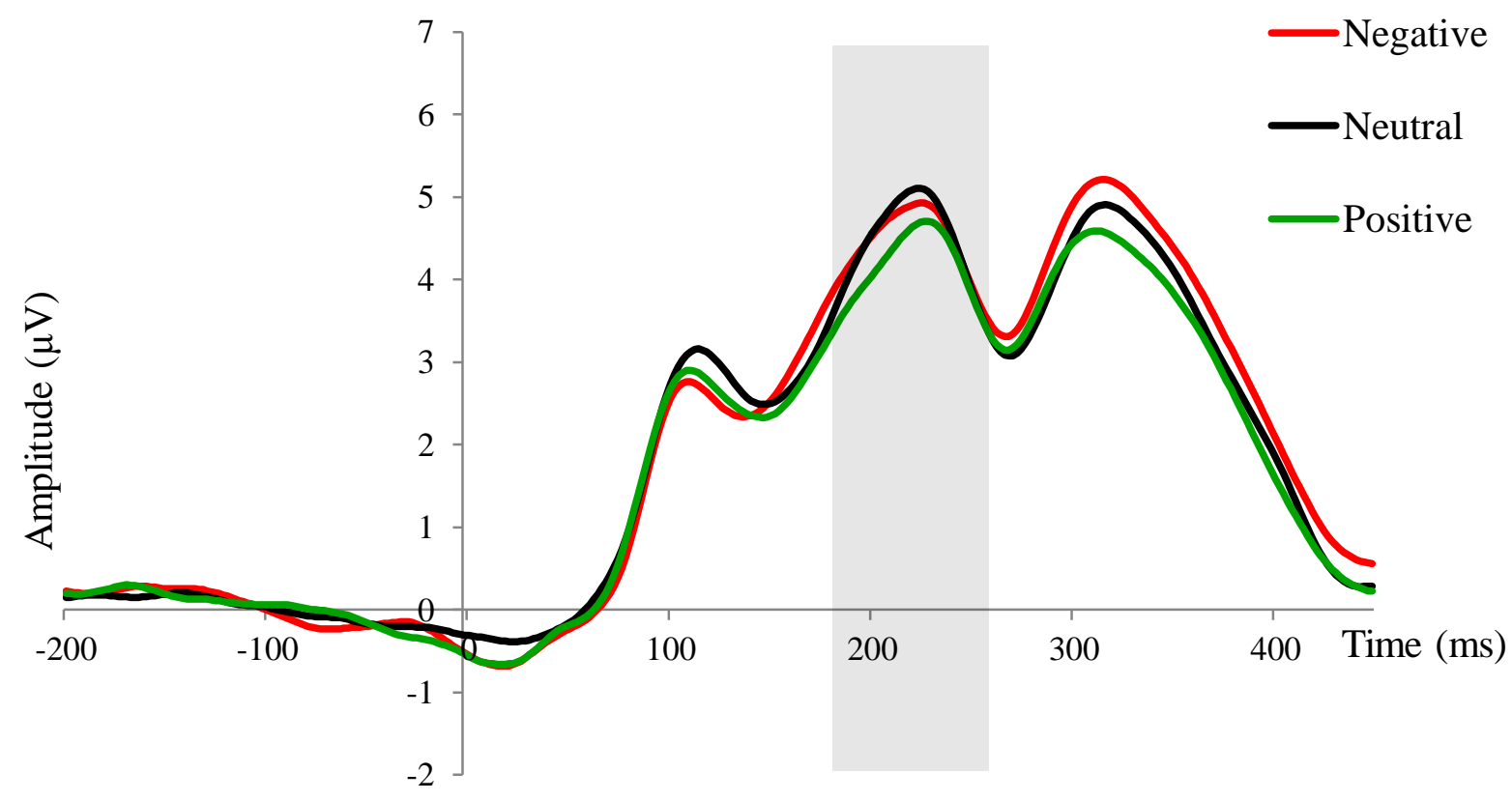

Figure 21. EPN amplitudes in the high distractor frequency condition collapsed across electrodes $(\mathrm{O} 1, \mathrm{OZ}, \mathrm{O} 2)$, by valence. There was no difference in EPN amplitudes elicited by neutral, positive and negative images.

Note. Shaded area represents timeframe for the EPN (180ms to 260ms) 


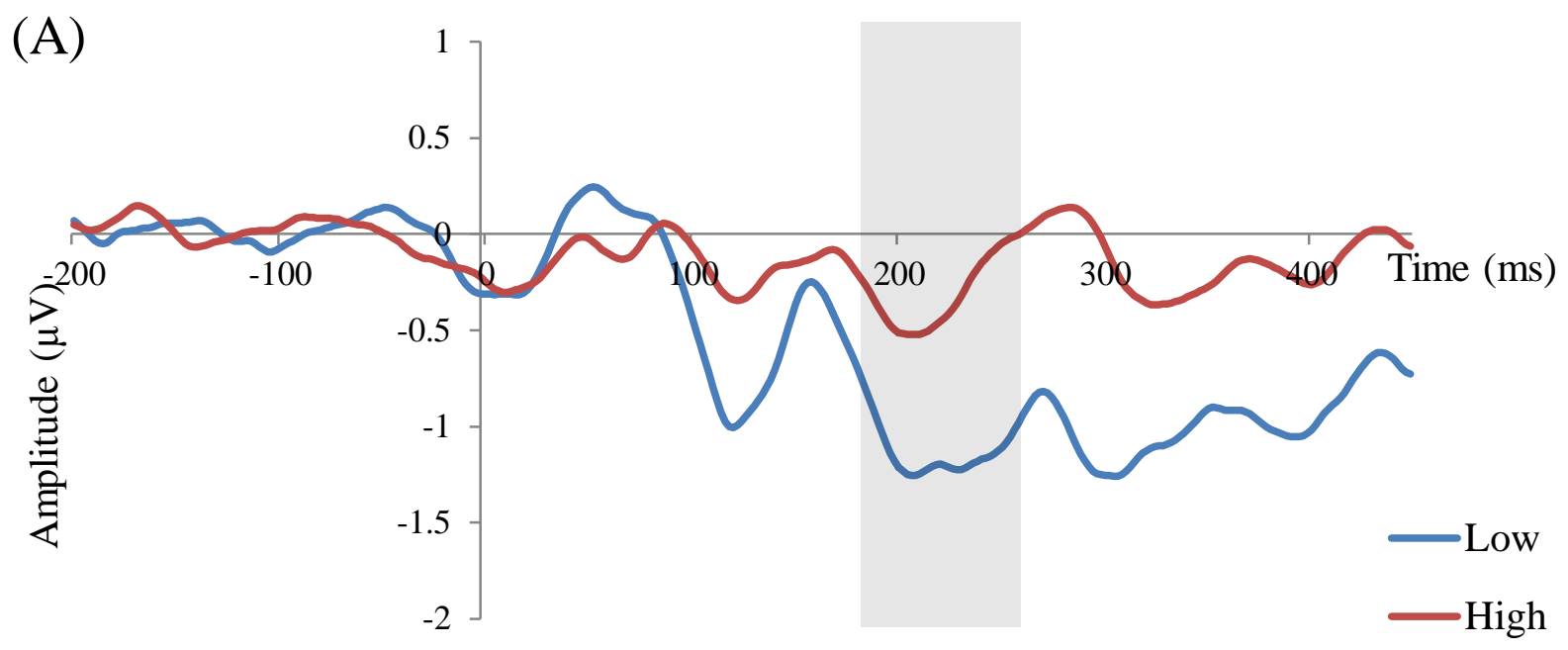

(B) Low

(C) High

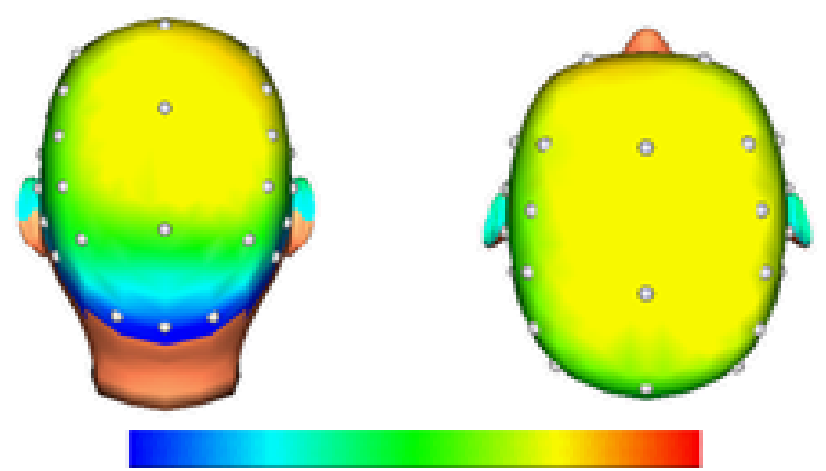

$-1.22$

0

1.22
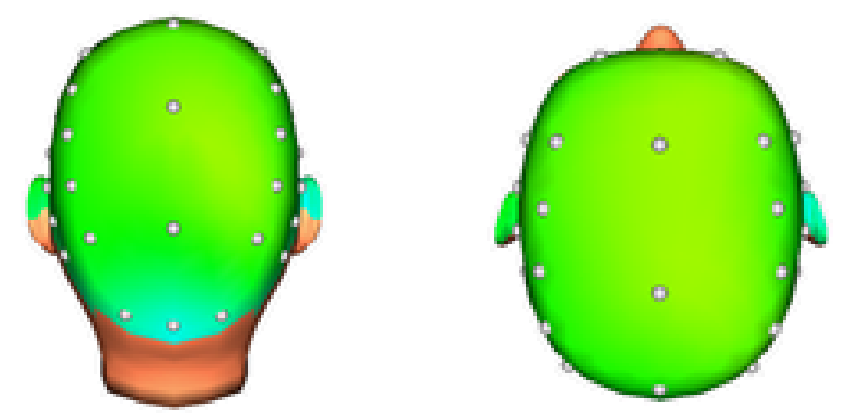

$-1.00$

0

1.00

Figure 22. (A) Difference waves (positive - neutral) for EPNs elicited by positive and neutral images, collapsed across electrodes $(\mathrm{O} 1, \mathrm{OZ}, \mathrm{O} 2)$, by distractor frequency condition. More negative values reflect a larger EPN for positive, compared to neutral, images. Scalp distribution for the positive-neutral difference are depicted for the low distractor frequency condition (B) and high distractor frequency condition (C).

Note. Shaded area in (A) represents timeframe for the EPN (180ms to 260ms)

Note. Head maps between distractor frequency conditions are presented on different scales. Scales were chosen to allow detailed representation of the positive-neutral difference. 


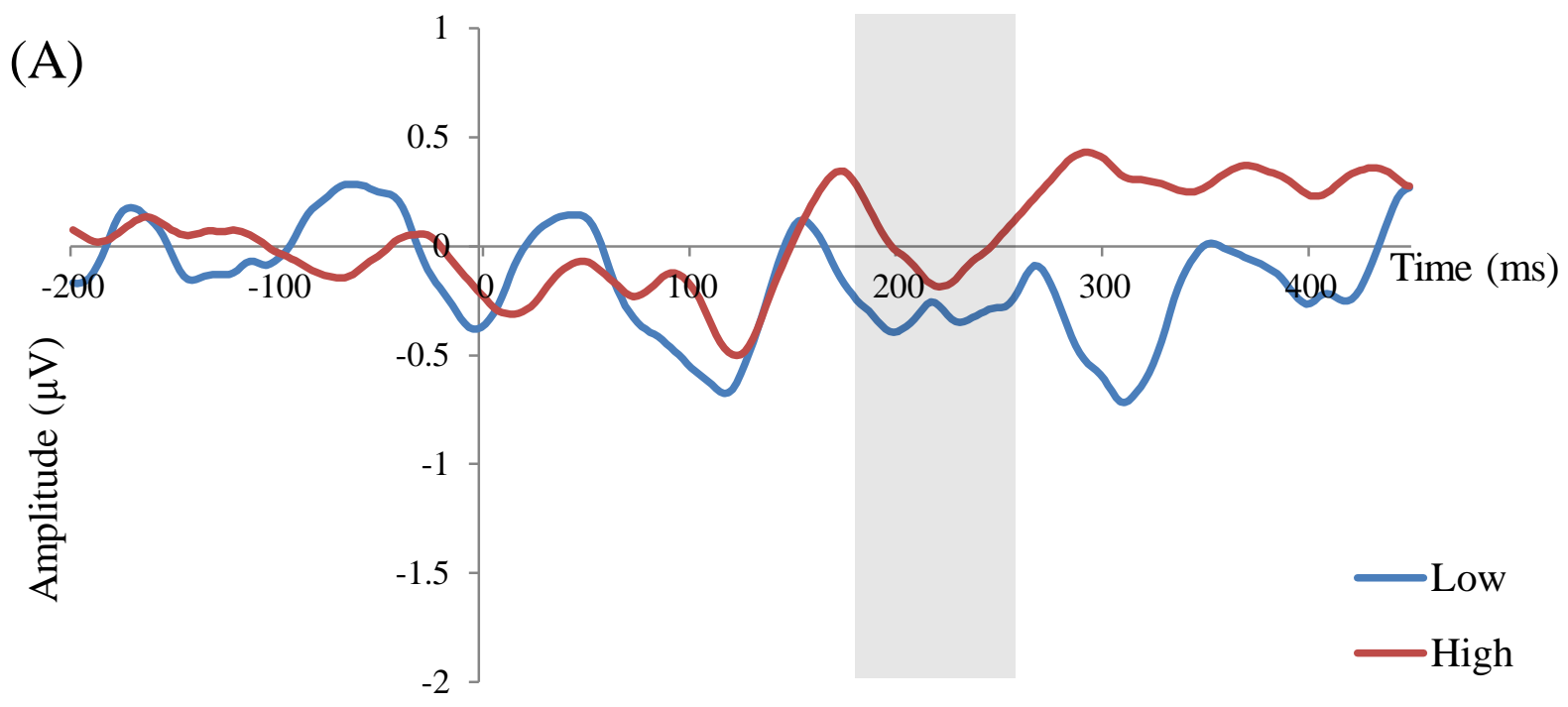

(B) Low
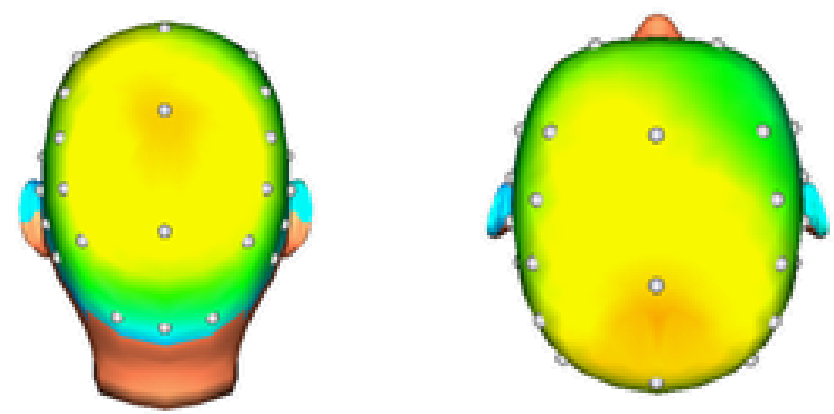

(C) High

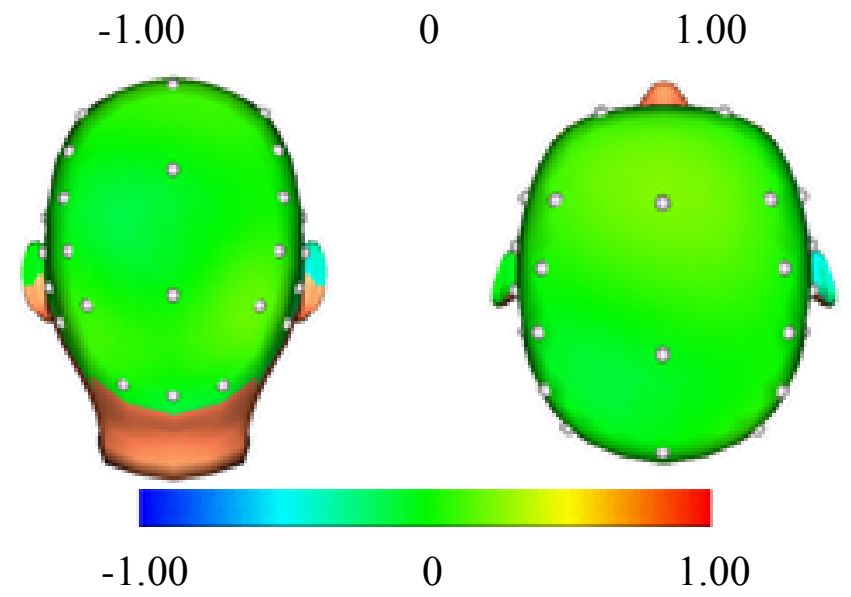

Figure 23. (A) Difference waves (negative - neutral) for EPNs elicited by negative and neutral images, collapsed across electrodes $(\mathrm{O} 1, \mathrm{OZ}, \mathrm{O} 2)$, by distractor frequency condition. More negative values reflect a larger EPN for negative, compared to neutral, images. Scalp distribution for the negative-neutral difference are depicted for the low distractor frequency condition (B) and high distractor frequency condition (C).

Note. Shaded area in (A) represents timeframe for the EPN (180ms to 260ms) 


\section{Anterior N2 (225 to $255 \mathrm{~ms})$.}

The anterior N2 waveforms for negative, neutral and positive images are presented in Figure 24 for the low distractor frequency condition, and Figure 25 for the high distractor frequency condition. For mean anterior N2 amplitudes and standard deviations, collapsed across electrode, by distractor frequency and valence, see Table 14. Anterior N2 amplitudes were entered into a 2 (distractor frequency: high, low) x 3 (valence: negative, neutral, positive) x 3 (electrode: F3, FZ, F4) mixed ANOVA. A main effect of electrode was observed, $F(1.68,102.43)=15.96, p<.001, \eta_{p}{ }^{2}=.21$, indicating that mean activity was greater at F3 and FZ than F4. There was also a marginal electrode $x$ valence interaction, $F(2.75,167.53)=2.26, p=.063, \eta_{p}{ }^{2}=.04$. However, this marginal interaction was not followed up, as it was not predicted nor relevant to hypotheses. A main effect of distractor frequency was observed $F(1,61)=4.35, p=.041, \eta_{p}{ }^{2}=.06$. As expected there was an enhanced (i.e., more negative) anterior N2 under low distractor frequency conditions than under high distractor frequency conditions. This supports the argument that the anterior N2 indexes reactive control processes. There was also a main effect of valence, $F(2,122)=6.98$, $p=.001, \eta_{p}{ }^{2}=.10$ and a significant quadratic effect of valence, $F(1,61)=10.57, p=.001, \eta_{p}{ }^{2}$ $=.15$, that were both qualified by a marginal valence $\mathrm{x}$ distractor frequency interaction, $F(2$, $122)=2.61, p=.08, \eta_{p}{ }^{2}=.041$, and a significant valence $\mathrm{x}$ distractor frequency quadratic, $F(1,61)=4.81, p=.032, \eta_{p}{ }^{2}=.07$. Data were collapsed across electrode for the following analyses.

Table 14

Mean (SD) anterior N2 amplitudes $(\mu V)$, collapsed across electrode, by distractor frequency and valence.

\begin{tabular}{lcc}
\hline & $\begin{array}{c}\text { Low Distractor } \\
\text { Frequency }\end{array}$ & $\begin{array}{c}\text { High Distractor } \\
\text { Valence }\end{array}$ \\
Negative & $-7.52(6.20)$ & Mrequency \\
Neutral & $-8.54(6.82)$ & $-4.87(4.21)$ \\
Positive & $-6.42(6.04)$ & $-4.97(4.36)$ \\
\hline
\end{tabular}

To follow-up this two-way interaction, repeated measures one-way ANOVAs with valence (negative, neutral, positive) as the within subjects variable were conducted on anterior N2 amplitudes for the high and low distractor frequency conditions separately. In the 
low distractor frequency condition, there was a significant main effect of valence, $F(2,62)=$ $6.60, p=.003, \eta_{p}^{2}=.18$, and a significant quadratic relationship for valence, $F(1,31)=$ $10.48, p=.003, \eta_{p}{ }^{2}=.25$. As depicted in Figure 24, anterior N2 amplitudes were largest for neutral images, smallest for positive images, and intermediate for negative images. Follow-up tests showed that anterior N2 amplitudes were significantly larger for neutral than positive images, $t(31)=3.97, p<.001, d=.33$, and marginally larger for negative than positive images, $t(31)=1.82, p=.079, d=.18$. However, no significant difference was observed between negative and neutral images, $t(31)=1.68, p=.104$.

Under high distractor frequency conditions, no effect of valence was observed (see Figure 25). The finding that the anterior N2 was modulated by emotion in the low, but not the high, distractor frequency condition is not surprising. It suggests that when reactive control is engaged, emotional characteristics of distractors are processed. However, the direction of modulation in the low distractor frequency condition (i.e., the fact that the anterior N2 was larger for neutral than positive or negative images) is somewhat counterintuitive as it suggests that more reactive control resources are being used to control neutral, than emotional, distraction. This is at odds with the typical finding that emotional distractors are more potent than neutral distractors. Possible interpretations of this effect will be raised in the general discussion. 


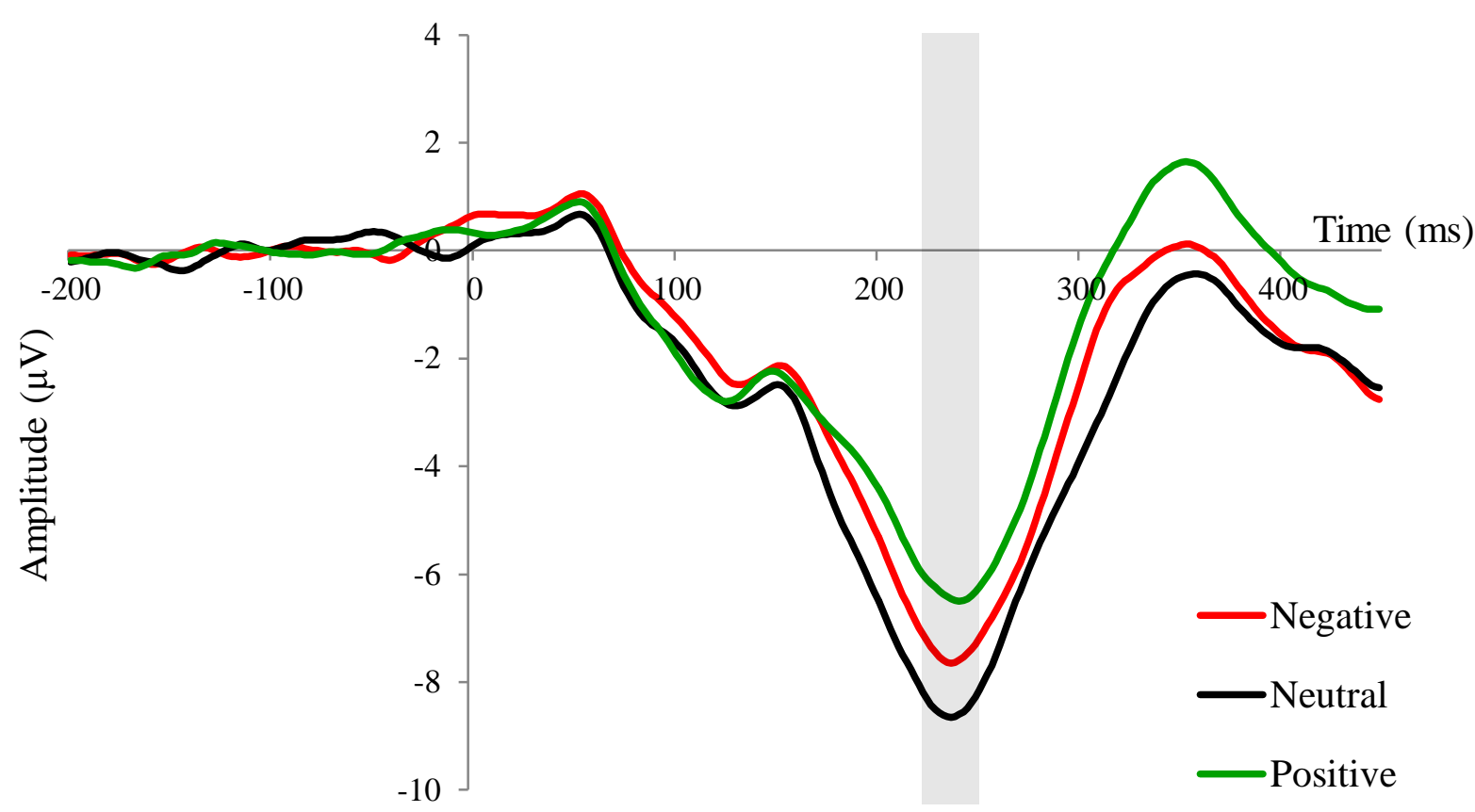

Figure 24. Anterior N2 amplitudes in the low distractor frequency condition, collapsed across electrodes (F3, FZ, F4), by valence. Neutral images elicited a larger (i.e., more negative) N2 than positive images. Negative images elicited a marginally larger anterior N2 than positive images. There was no difference in anterior N2 amplitudes elicited by neutral and negative images.

Note. Shaded area represents timeframe for the anterior N2 (225ms to $255 \mathrm{~ms})$

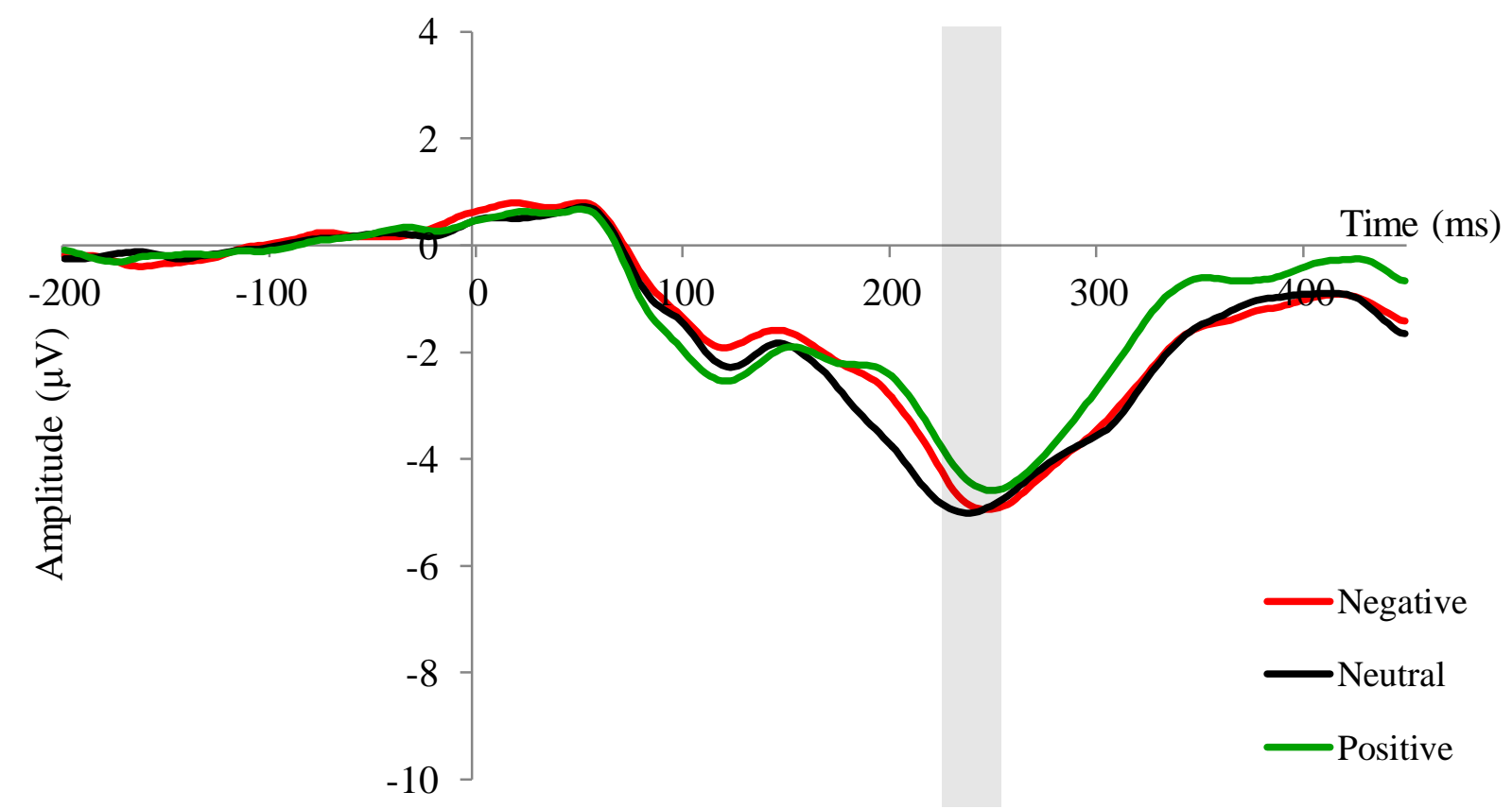

Figure 25. Anterior N2 amplitudes for in the high distractor frequency condition, collapsed across electrodes (F3, FZ, F4), by valence. There was no difference in anterior N2 amplitudes elicited by neutral, positive and negative images.

Note. Shaded area represents timeframe for the anterior N2 (225ms to 255ms) 


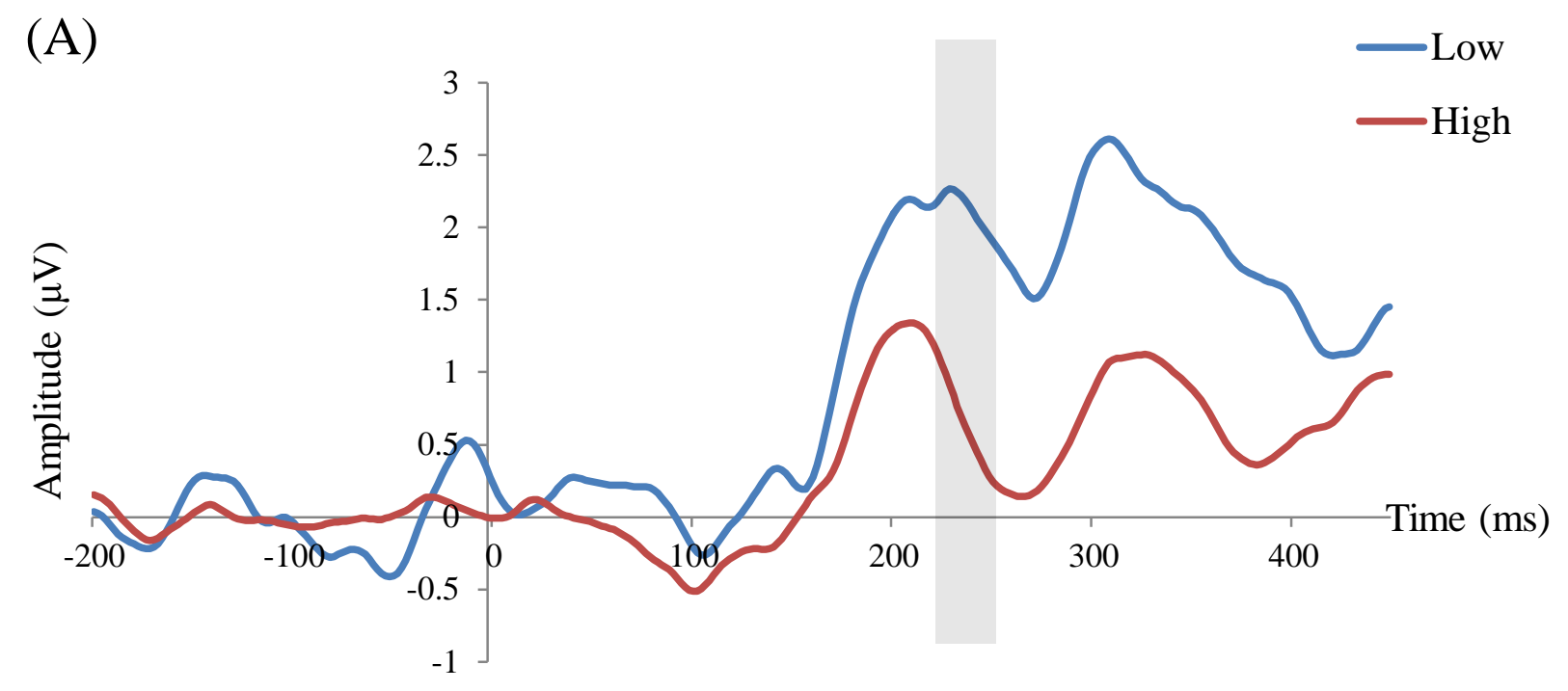

(B) Low

(C) High
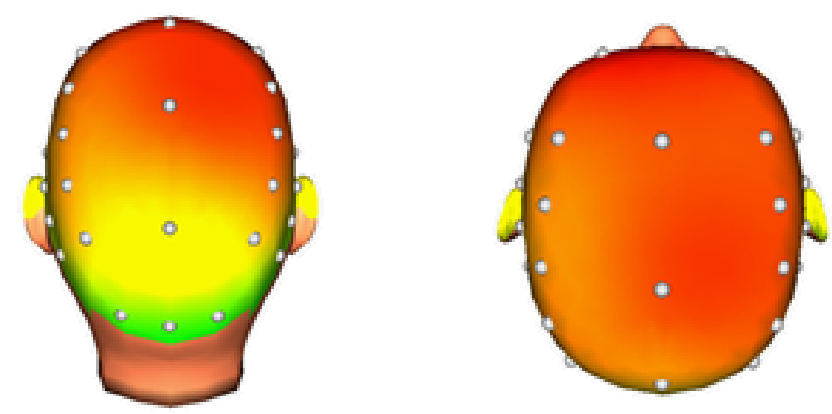

$-2.45$

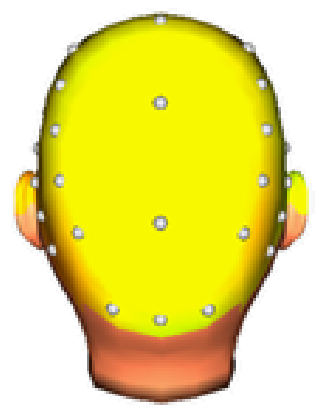

$-1.00$
0

2.45

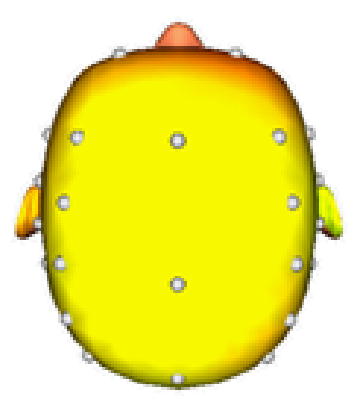

1.00

Figure 26. (A) Difference waves (positive - neutral) for anterior N2s elicited by positive and neutral images, collapsed across electrodes (F3, FZ, F4), by distractor frequency condition. More positive values reflect a smaller anterior N2 for positive, compared to neutral, images. Scalp distribution for the positive-neutral difference are depicted for the low distractor frequency condition (B) and high distractor frequency condition (C).

Note. Shaded area in (A) represents timeframe for the N2 (225ms to $255 \mathrm{~ms})$

Note. Head maps between distractor frequency conditions are presented on different scales. Scales were chosen to allow detailed representation of the positive-neutral difference. 


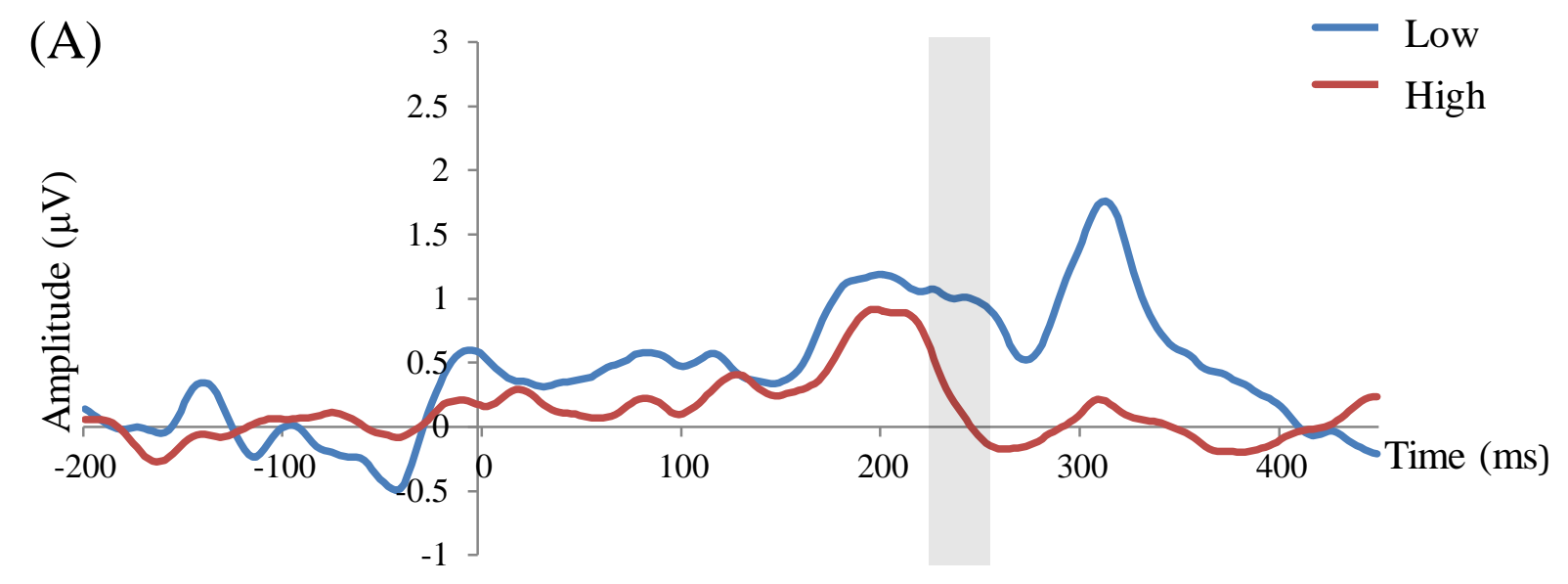

(B) Low

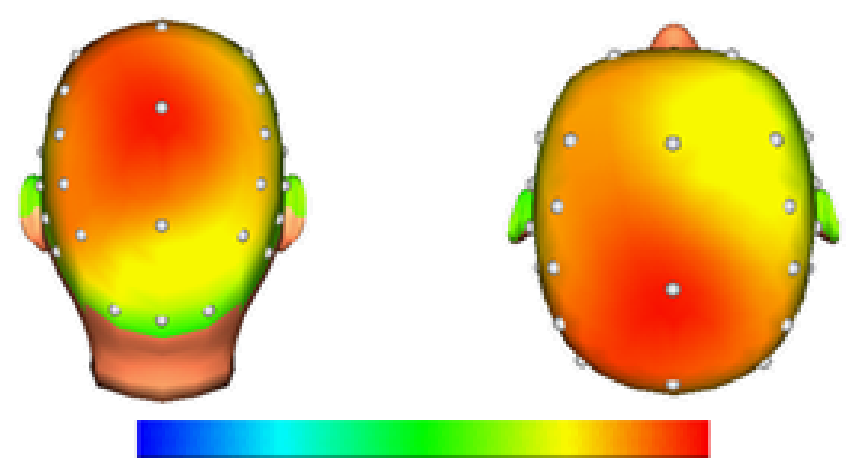

$-1.56$

$0 \quad 1.56$

(C) High
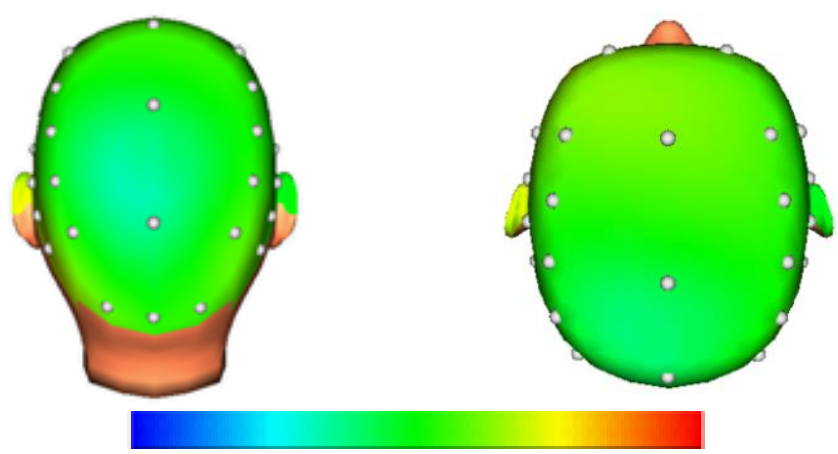

$-1.00$

0

1.00

Figure 27. (A) Difference waves (negative - neutral) for anterior N2s elicited by negative and neutral images, collapsed across electrodes (F3, FZ, F4), by distractor frequency condition. More positive values reflect a smaller anterior N2 for negative, compared to neutral, images. Scalp distribution for the negative-neutral difference are depicted for the low distractor frequency condition (B) and high distractor frequency condition (C). Note. Shaded area in (A) represents timeframe for the N2 (225ms to $255 \mathrm{~ms})$ Note. Head maps between distractor frequency conditions are presented on different scales. Scales were chosen to allow detailed representation of the negative-neutral difference. 


\section{Summary}

In Experiment 2 I examined the mechanisms by which proactive control minimises emotional distraction. The behavioural data mirrored Experiment 1. Emotional distraction was more pronounced under low, compared to high, distractor frequency conditions. Indeed there was no emotional distraction effect (i.e., emotional images were no more distracting than neutral images) at all in the high distractor frequency condition, suggesting that proactive mechanisms can control emotional distraction as effectively as neutral distraction. However, while the pattern of data was similar for the subset of participants included in ERP analyses (see Figure 13), the interaction between valence and distractor frequency did not reach conventional levels of significance for that group $(p=.104)$.

Under low distractor frequency conditions (in which reactive control is assumed to dominate) there was typical emotional modulation of the LPP; a pronounced positivity was seen for both negative and positive images, compared to neutral images (see Figure 16). Further, the expected modulation of the EPN by positive images was observed (see Figure 20). However, inconsistent with predictions, the EPN was not modulated by negative images. While this could be interpreted as negative images not receiving prioritised perceptual processing relative to neutral images, the pronounced LPP for negative images refutes this idea. Thus, it is more likely that the EPN was simply not a sensitive measure for early negative distractor processing in the present study.

Under high distractor frequency conditions (in which proactive control is assumed to dominate), the LPP was modulated by positive, but not by negative, images (see Figure 17). This suggests that proactive control reduces sustained attention to negative distractors, but (unexpectedly) does not entirely prevent sustained processing of positive distractors. Further, the EPN was not modulated by either negative or positive images (see Figure 21). As the EPN was not modulated by negative images under low distractor frequency conditions, the absence of an EPN for negative images under high distractor frequency conditions cannot be interpreted in the context of my predictions. The elimination of the EPN for positive images under high distractor frequency conditions suggests that proactive control minimises early perceptual processing of positive distractors. However, this conclusion is complicated by the presence of an LPP to positive images in the same high distractor frequency. If proactive control abolishes emotional processing of images from early stages, then no LPP should be observed.

Consistent with predictions, the anterior N2 was more pronounced under low, than high, distractor frequency conditions. This supports the argument that it indexes reactive 
control processes. The anterior N2 was further modulated by valence of the distractor under low distractor frequency conditions. Rather paradoxically, amplitudes were largest for neutral distractors, and smallest for positive distractors (see Figure 24), suggesting that more reactive control resources are used to control the relatively less potent neutral distractions.

It is important to note that a number of the results in regards to the ERP components of interest were statistically marginal. While only predicted marginal effects were followed up, the findings from Experiment 2 should be interpreted with caution. Indeed, one primary limitation of this experiment is that the data from the ERP experiment were very noisy. Modulation of the EPN and LPP were expected to be small because images (distractors) were presented peripherally outside the focus of attention and were task-irrelevant. These factors are known to reduce the magnitude of the LPP and EPN (e.g., De Cesarei \& Codisopti, 2006; Norberg \& Wiens, 2013; Schupp et al., 2007; Wiens et al., 2011, 2012). However, as this is the first study to look at the LPP and EPN components in this particular emotional distraction task, I was not able to estimate effect sizes (and therefore sample sizes) ahead of time. Further, I was not able to accurately estimate the number of trials needed per condition to produce a stable mean for each participant. I included what is usually considered a reasonable number of participants, and a reasonable number of trials per condition for an ERP study examining the EPN and LPP. However, it is clear that this study should be followed up in larger samples with more trials per condition. Although much of the noise in the data likely comes from individual differences (e.g., variation in cognitive control strategy used and susceptibility to emotional distraction), increasing trial numbers and the sample size should increase the signal to noise ratio of the experiment. More power would allow a more thorough exploration of the LPP and EPN under emotional distraction conditions, and stronger conclusions could be made.

\section{General Discussion}

In order to achieve goals, attention to irrelevant distractions must be controlled. Control over distractions can be achieved via proactive or reactive cognitive control strategies (Braver, 2012). It has been established that proactive strategies are more effective than reactive strategies at controlling neutral distraction (Braver, 2012; Chao, 2011, Lee \& Chao, 2012; Munneke et al., 2008; Padmala \& Pessoa, 2011; Van der Stigchel \& Theeuwes, 2006). However, whether proactive mechanisms can control attention to entirely irrelevant emotional distractors as effectively as neutral distractors is not clear. In this thesis I examined cognitive control over emotional distraction. In Experiment 1, I tested whether proactive mechanisms can control attention to irrelevant emotional distractors as effectively as 
irrelevant neutral distractors. Participants completed an emotional flanker task under low perceptual load conditions. Target letters were presented at fixation, and distractors (positive, negative or neutral images) appeared above or below the letter display. Distractors were presented on either a low proportion (25\%) or high proportion (75\%) of trials to evoke reactive and proactive control strategies, respectively. In Experiment 2, I used ERPs to examine how proactive mechanisms control irrelevant emotional distraction. EEG was recorded while participants completed a slightly modified version of the distraction task from Experiment 1.

\section{Behavioural Results}

In Experiment 1, emotional images were more distracting than neutral images when presented on a low proportion of trials (i.e., under reactive control conditions). However, when distractors were frequent (i.e. under proactive control conditions), this emotional distraction effect was markedly attenuated - emotional images were only slightly more distracting than neutral images. Follow-up analyses indicated that the reduction in emotional distraction under high distractor frequency conditions was not due to habituation (i.e., image repetition). The attenuation of emotional distraction under high distractor frequency conditions suggests that proactive mechanisms can control attention to emotional distractors almost as effectively as neutral distractors.

In Experiment 2, I replicated the behavioural results of Experiment 1 using a similar (but not identical) experimental design. In line with the findings of Experiment 1, emotional images were more distracting than neutral images under low distractor frequency conditions. This emotional distraction effect was completely abolished under high distractor frequency conditions - i.e., there was no difference between emotional and neutral distraction. In Experiment 1, a concern was that the interaction between emotional distraction, and distractor frequency, was due to a floor effect (no neutral distraction was observed in the high distractor frequency condition). However, this explanation cannot account for the pattern of behavioural data in Experiment 2. In Experiment 2 there was a significant (but small) distraction effect under high distractor frequency conditions that was not modulated by valence. Emotional and neutral distractions were controlled equally as effectively under proactive control conditions, in the absence of a floor effect for neutral distraction.

A noteworthy finding of the current experiments was that emotional images (both positive and negative) were more distracting than neutral images under reactive control conditions. Broadly, there has been debate as to whether entirely irrelevant emotional distractors are more potent than irrelevant neutral distractors. Some studies have found no 
evidence for task-irrelevant emotional distraction effects (Barratt \& Bundesen, 2012; Lichtenstein-Vidne et al., 2012; Okon-Singer et al., 2007). Other studies have found evidence for irrelevant negative (but not positive) emotional distraction effects (Fox et al., 2012; Yates et al., 2010). Indeed, only one study (Grimshaw et al., in prep) has found evidence for both positive and negative emotional distraction effects when distractors are entirely irrelevant. My studies provide a valuable contribution to the literature, demonstrating that entirely irrelevant emotional images are more potent distractors than irrelevant neutral images in two independent experiments. Further, my studies suggest that emotional distraction effects are equivalent for positive and negative distractors (with matched subjective arousal ratings).

While my studies show that entirely irrelevant emotional distractors are more potent than neutral distractors, they also demonstrate that irrelevant emotional distraction can be controlled - we are not doomed to always experience emotional distraction. Emotional and neutral distraction levels were similarly low under high distractor frequency conditions, suggesting that proactive mechanisms can control entirely irrelevant emotional distractions both positive and negative - as effectively as irrelevant neutral distractions. These results extend those of Padmala and Pessoa (2014) who found that proactive mechanisms controlled negative emotional distractions as effectively as neutral distractions when distractors were presented in an attentionally prioritised location (fixation; Beck \& Lavie, 2005). My studies build on the study conducted by Padmala and Pessoa (2014) by showing that both positive and negative distractions can be effectively controlled, even when presented in irrelevant locations. Theoretically, the clear attenuation of emotional (compared to neutral) distraction under proactive control conditions supports the argument that emotional attention can be modulated by top-down control systems (e.g., Pessoa et al., 2002; Pessoa, 2009; Pourtois et al., 2013), and is inconsistent with the argument that emotional attention is independent of top-down control systems (e.g., Brosch et al., 2011; Viviani, 2013; Vuillumier, 2005).

The finding that emotional distraction can be abolished under conditions that evoke proactive control may help to explain why some previous studies find that irrelevant emotional distractors are no more potent than irrelevant neutral distractors (e.g., Barratt \& Bundesen, 2012; Lichtenstein-Vidne et al., 2012; Okon-Singer et al., 2007). These previous emotional distraction studies present distractors on either every trial, or a very high proportion of trials. The current studies clearly indicate that when distractors are frequent, emotional distraction does not differ (or only differs very slightly) to neutral distraction. It is thus not surprising that previous studies did not find evidence for irrelevant emotional distraction because they used tasks in which distractors were presented frequently. Indeed, it 
is perhaps more surprising that some studies have found evidence for irrelevant emotional distraction even when distractors were presented on every trial (e.g., Fox et al., 2012; Yates et al., 2010).

One possible reason that two previous studies (Fox et al, 2012; Yates et al., 2010) found an emotional distraction effect under high distractor frequency conditions could be that their paradigms did not evoke proactive control (i.e., participants used reactive control strategies). In both of these previous studies, aversively conditioned angry faces, nonconditioned angry faces and neutral faces were used as distractors. There is evidence that aversively conditioned stimuli are more distracting (as demonstrated by Fox et al., 2012; and Yates et al., 2010), and elicit larger amygdala responses (Lim, Padmala \& Pessoa, 2008), than non-conditioned stimuli, suggesting that they receive more attentional gain from the emotional attention systems. It is possible that suppressing aversively conditioned (compared to non-conditioned) stimuli is more effortful, and taxes proactive control resources to a greater extent. Presenting distractors frequently may not be motivating enough to evoke proactive control strategies when some of the distractors have been aversively conditioned (the effort might not be 'worth it'). If so, then Fox et al., (2012) and Yates et al., (2010) may simply not have evoked proactive control in their paradigms, meaning that participants used reactive control strategies. If participants used reactive control strategies, then it is not so surprising that emotional distractors were more potent than neutral distractors. An incentive to use proactive control over and above presenting distractors frequently (e.g., providing rewards; Locke \& Braver, 2008; Padmala \& Pessoa, 2011, 2014; Pessoa \& Engelmann, 2010) may be necessary when distractors have been aversively conditioned.

\section{Differences in Behavioural Results across Experiments.}

Despite similarities across studies, there were also a few differences in the pattern of results. First, RTs were overall much slower in the high, than the low, distractor frequency condition in Experiment 2, but not in Experiment 1. A follow-up analysis indicated that RTs were slower right from the beginning of the experiment (i.e., the effect did not develop across time). Perhaps the simplest explanation for this difference is that, despite random assignment, the high distractor frequency condition comprised more slow responding participants than the low distractor frequency condition in Experiment 2. Alternatively, the slowing could have been caused by the effort used to maintain proactive control across a sustained period of time. Proactive control is associated with sustained maintenance of activity in the lateral prefrontal cortex (Braver, et al., 2014), and is argued to be an effortful process (Braver, 2012). It is plausible that a compensatory slowing in responding is necessary in order to maintain 
proactive control (i.e., sustained activation of areas in the prefrontal cortex) over a long period of time. The distraction task in Experiment 2 was almost twice as long as Experiment 1 (720 trials versus 384 trials). Participants were aware of both the experiment length, and the frequency at which distractors would appear, before beginning the experiment. It could be that simply knowing the length of the experiment engaged mechanisms that slowed overall responding to enable the maintenance of proactive control for the length of the experiment. Yet another plausible explanation is that the slowing is strategic - proactive control could be achieved by being vigilant for distractors, which caused slowing on all trials. However, if this were the case then an equal slowing effect should have been observed in Experiment 1. It is possible that participants across the two experiments adopted different proactive control strategies. Classifying control as proactive or reactive doesn't mean there are just two ways to institute control. Indeed the idea that there are multiple ways (mechanisms) to achieve proactive control is interesting and might account for differential effects of proactive control on behaviour under different experimental conditions, or in different participant populations.

A second difference between experiments was that about half as much distraction was observed in the reactive control condition in Experiment 2 compared to Experiment 1. Despite the overall decrease in distraction, emotional distractors were about twice as distracting as neutral distractors in each experiment. One plausible explanation for the overall decrease in distraction is that participants in Experiment 2 may have been more motivated to do well on the task than participants in Experiment 1. There are a few differences between experiments that could have led to this change in motivation. First, the sample in Experiment 2 consisted of both first year psychology students (like the sample of Experiment 1), but also self-selected community participants who were given vouchers for their participation - and thus, were likely a more motivated group of participants overall. Second, in Experiment 2 participants were run individually (as opposed to in groups of four as in Experiment 1), and due to the lengthy nature of preparing participants for EEG recording, had time to build a rapport with the experimenter before beginning the task. This rapport likely increased their motivation to do well on the task for the experimenter. Importantly, increasing motivation (i.e., providing incentives) is known to evoke proactive control strategies (Locke \& Braver, 2008; Padmala \& Pessoa, 2011, 2014; Van den Berg et al., 2014), as well as increase the effectiveness of reactive control strategies (Hu, Padmala \& Pessoa, 2013). The decrease in distraction observed in the low distractor frequency condition in Experiment 2 (compared to Experiment 1) could have been caused by increased use of proactive control strategies (even 
in the 'reactive' control condition), or an overall increase in the effectiveness of reactive control strategies.

\section{The Blocking of Distractors.}

In the distraction tasks used in the current studies, the valence of distractors was blocked such that only positive, negative or neutral images appeared in a given block. Due to the blocked design, there are two possible explanations for why there was pronounced distraction from emotional (compared to neutral) images under reactive control conditions. It is possible that the actual emotionality of a distractor on a given trial underpinned the emotional distraction effect. Alternatively, the emotional distraction effect could have been driven by the expectation that a distractor would be (or could be) emotional. Knowing distractors are emotional in a given block could prime one to monitor all distractors. Previous studies have found that indicating the valence of an upcoming emotional distractor increases distraction (Kleinsorge, 2007, 2009), suggesting that knowing a distractor will be emotional primes you to monitor it more closely. Further, Devue, Belopolsky and Theeuwes (2011) found that spider-phobics were distracted by pictures of butterflies (i.e., neutral distractors) when they were presented intermixed with pictures of spiders (i.e., emotional distractors), suggesting that when any distractor could be emotional, all distractors were monitored and were thus equally distracting. However, when butterflies and spiders appeared in separate blocks of trials (i.e., spider-distractors were not expected to appear in blocks of butterflydistractors), only spider pictures were distracting. The study by Devue et al. (2011) suggests that blocking the valence of distractors may be necessary for emotional distraction effects (i.e., pronounced emotional over neutral distraction) to occur. To determine whether the expectation that a distractor could be emotional or the actual emotionality of a distractor on a given trial drives task-irrelevant emotional distraction effects, future studies could compare distraction in a blocked versus mixed (i.e., emotional and neutral distractors are presented randomly in the same block) design. If the expectation that any distractor might be emotional causes emotional distraction, then, when distractors are intermixed, emotional and neutral distraction should be equivalent, but both large (i.e., neutral distraction should increase to emotional levels). The logic of this prediction stems from the argument that every distractor (including neutral distractors) should be equally monitored as it could be emotional, and thus all distractors should be equally distracting.

\section{ERP Results}

The behavioural results of the current studies clearly demonstrate that proactive mechanisms can control emotional distraction as effectively as neutral distraction. However, 
the behavioural data cannot be used to address the question of how proactive control minimises emotional distraction. To address the question of mechanism, I examined ERP responses to the distracting images in Experiment 2. Before interpreting the ERP results, it is important to note that a number of predicted (and critical) interactions did not reach conventional levels of significance. For participants included in ERP analyses, emotional distraction did not interact with distractor frequency $(p=.104)$. However, the pattern of distraction was similar to that of all participants. Numerically, emotional distractors were only more potent than neutral distractors in the low (but not high) distractor frequency condition - consistent with the argument that proactive control eliminates emotional distraction effects. Follow-up analyses indicated that the increase in emotional distraction under low distractor frequency conditions (i.e., reactive control conditions) was particularly pronounced for positive emotional distractors. While the interaction between emotional distraction and distractor frequency wasn't statistically significant, there was a significant reduction in distraction from low to high distractor frequency conditions, suggesting that proactive control was effectively engaged. A number of ERP effects were also statistically marginal (but only predicted marginal interactions were followed up). In light of the marginal behavioural effects, and marginal ERP effects, I exercise caution interpreting the ERP results from Experiment 2.

\section{The EPN and LPP.}

As expected, when distractors were rare, a pronounced EPN was observed for positive images, and a pronounced LPP was observed for both positive and negative images, relative to neutral images (although it should be noted that differences in the EPN and LPP by distractor frequency condition were followed up based on marginal distractor frequency $\mathrm{x}$ valence quadratic interactions). These findings are consistent with the typical emotional modulation of the EPN and LPP (e.g., Hajcak et al., 2012; Hajcak \& Nieuwenhuis, 2006; Junghöfer et al., 2001; Schupp et al., 2006a; Weinberg \& Hajcak, 2010), and suggest that when reactive control dominates, emotional characteristics of both positive and negative distractors are processed. The emotional modulation of the EPN and LPP under reactive control conditions is consistent with the finding that emotional distractors cause more behavioural interference than neutral distractors under reactive control conditions. Unexpectedly, the EPN was not modulated by negative emotional images. This finding is particularly curious, as there was typical emotional modulation of the LPP by negative images - so it is not the case that emotional aspects of the negative images were simply not processed. Broadly, there is some evidence that the EPN is particularly sensitive to pleasant 
(over unpleasant) information, with the largest amplitudes generally elicited by erotic images (e.g., Hajcak et al., 2012; Schupp et al., 2004a, 2006a, 2006b; Schupp, Schmälzle, Flaisch, Weike \& Hamm, 2012; Weinberg \& Hajcak, 2010). My results add to this body of literature, and suggest that under the specific experimental conditions that were used here (i.e., images were presented peripherally outside the focus of attention and were task-irrelevant) the EPN is sensitive to erotic images, but not to images of mutilated bodies. As the EPN was not modulated by negative images, it could not be used as a reliable index of early allocation of attention to the perceptual processing of negative information.

Unexpectedly, proactive control had differential effects on ERPs elicited by negative and positive distractors. This finding is unexpected as proactive control had similar behavioural effects on positive and negative distraction. Due to this difference, I will discuss the effect of proactive control on ERP indices of distractor processing separately for positive and negative distractors.

\section{The effect of proactive control on negative distractor processing. Increasing} distractor frequency eliminated emotional modulation of the LPP by negative images. This attenuation of the LPP is consistent with predictions, and suggests that proactive control reduces sustained emotional attention to negative distractors. There was no emotional modulation of the EPN by negative distractors in the high distractor frequency condition. However, because the EPN did not index negative image processing under reactive control conditions, the finding that negative images did not modulate the EPN under proactive control conditions cannot be interpreted in the context of my predictions. It is impossible to know whether proactive control minimised early perceptual processing of negative distractors (which in turn reduced sustained processing of negative distractors), or whether proactive control only minimised sustained processing of negative distractors. Indeed, the question of how proactive mechanisms control negative emotional distraction cannot be answered in my experiment.

\section{The effect of proactive control on positive distractor processing. Increasing} distractor frequency eliminated emotional modulation of the EPN by positive images. This is a particularly important finding as it directly speaks to my research question - that is, 'how does proactive control minimise emotional distraction?'. The elimination of the EPN in the high distractor frequency condition suggests that proactive control minimises emotional distraction by reducing early allocation of attentional resources to perceptually process positive distractors. Curiously, despite not eliciting an EPN, positive images elicited a pronounced LPP under high distractor frequency conditions. The finding that proactive 
control suppressed the EPN but not the LPP elicited by positive images is unexpected to say the least. If we accept that the EPN is a valid index of early perceptual processing of a positive stimulus, then perhaps this finding requires some re-interpretation of the LPP effect. Indeed, it would be difficult to sustain attention to a stimulus that did not preferentially recruit attentional resources. The LPP is affected by a number of higher-level cognitive processes - e.g., reappraising stimuli (Hajcak \& Nieuwenhuis, 2006), and intentional suppression of emotional responses (Moser, Hajcak, Bukay \& Simons, 2006). Further, the LPP is sustained after a stimulus has disappeared (Hajcak \& Olvet, 2008), and the magnitude of the LPP is related to memory performance (Dolcos \& Cabeza, 2002; Koenig \& Mecklinger, 2008). Taken together, these previous findings suggest that the LPP is driven by sustained attention to an internal representation of an event and is affected by voluntary processes. With this in mind, it could be that the LPP elicited by positive images in the high distractor frequency condition was produced independently of active perceptual processing of positive images. The valence of distractors was blocked, so the participants expected positive distractors to appear; every trial brought the possibility of an erotic image. The onset of the letter display may have triggered thoughts of erotic images (which produced an LPP), even if the erotic image was not perceptually processed. This interpretation provides a possible explanation for why you could get attenuation of early emotional modulation (i.e., no EPN), but evidence of late emotional processing (i.e., an LPP).

One way of determining whether the LPP is elicited independent of active image processing would be to compare the LPP on distractor-present and distractor-absent trials. If the LPP elicited in blocks with frequent erotic distractors reflects sustained erotic processing, whether an image is present or not, then the magnitude of the LPP should be comparable on distractor-present and distractor-absent trials. A method of testing whether the LPP reflects sustained erotic processing because of the expectation that an erotic image will appear would be to compare LPP magnitudes in a blocked versus intermixed design. When the valence of distractors is intermixed, the onset of the trial does not necessarily mean an erotic image will be presented - the image could be positive, negative or neutral. Intermixing the valence of distractors eliminates the expectation that a distractor will be erotic. If the LPP reflects sustained erotic processing (that is independent of active image processing) because it is expected that an erotic image will appear, then erotic images should elicit pronounced LPPs when distractors are blocked, but not intermixed.

A second possible explanation for the finding that frequent positive distractors elicited an LPP, but not an EPN, is that proactive control reduced emotional processing of distractors 
to the extent that they did not disrupt the primary goal (e.g., finding the target), but some emotional processing of positive distractors still occurred that was not indexed by the EPN. If so, then emotional modulation of the LPP by positive images should be less pronounced under high, than low, distractor frequency conditions. The logic of this prediction stems from the argument that proactive control should at least partially attenuate the LPP to positive images (so that they are less distracting) in order to focus attentional resources on the task at hand. Inconsistent with this idea, results indicated that there was no significant difference in the magnitude of the LPP elicited by positive (compared to neutral) images under high and low distractor frequency conditions. This does not rule out the possibility that the LPP was attenuated in the high distractor frequency condition. It may be that the LPP was attenuated, but my experiment did not have enough power to pick up this small decrease in LPP amplitude. Indeed, visual inspection of the difference waves (see Figure 18A) shows that the LPP for positive images was quantitatively larger under low, than high, distractor frequency conditions. This emphasises the need to replicate Experiment 2 with more trials and participants to increase statistical power to detect such differences if they exist.

\section{The Anterior N2}

In Experiment 2, a third ERP component, the anterior N2, was examined to determine whether it would index cognitive control strategy - i.e., distinguish between the use of proactive and reactive control strategies. Consistent with the argument that the anterior N2 reflects reactive control processes (and its typical manifestation in paradigms that evoke reactive control; e.g., Eimer, 1993; Jodo \& Kayama, 1992; Kok, 1986; Pfefferbaum et al., 1985), it was more pronounced under low, than high, distractor frequency conditions. Interestingly, under low distractor frequency conditions, the anterior N2 was modulated by valence of the distractor. Anterior N2 amplitudes were significantly larger for neutral images than positive images. While neutral images produced quantitatively larger anterior N2 amplitudes than negative images, this difference was not statistically significant.

There are a few possible interpretations of the finding that image valence modulated the anterior N2. Some researchers have argued that the magnitude of the anterior N2 reflects the amount of cognitive conflict, with larger amplitudes reflecting more conflict (see Nieuwenhuis et al., 2003). However, this cognitive conflict account does not fit well with the present data, as it would suggest that neutral distractors are more conflicting than emotional distractors. We know that emotional distractors are more potent, and produce more behavioural interference, than neutral distractors (Fox et al., 2012; Grimshaw et al., in prep; Yates et al., 2010), so they should logically also produce more cognitive conflict than neutral 
distractors. While the behavioural emotional distraction effect was not statistically significant for ERP subjects, the pattern of data was not at all indicative of greater neutral (than emotional) distraction under low distractor frequency conditions.

The attenuation of the anterior $\mathrm{N} 2$ by emotional images in the low distractor frequency condition could simply suggest that emotional distractions were not well controlled by reactive strategies. Larger magnitudes of the anterior $\mathrm{N} 2$ have been associated with more successful response inhibition (Eimer, 1993; Jodo \& Kayama, 1992; Kok, 1986; Pfefferbaum, et al., 1985). Extending this notion beyond response inhibition, it is possible that larger amplitudes of the anterior N2 reflect better control of conflict or distraction. By this logic, the enhanced anterior N2 in the presence of neutral, compared to emotional, distractors in the current study would be interpreted as more successful reactive control over neutral distraction than emotional distraction. Indeed, neutral distractors produce less distraction than emotional distractors under reactive control conditions suggesting that they are better controlled. Whether the better control of neutral (compared to emotional) distractions is due to decreased effort in monitoring for, and therefore controlling, emotional distractions (as would be consistent with a conflict monitoring hypothesis of the anterior N2; see Nieuwenhuis et al., 2003), or simply due to decreased ability to control emotional distractions is an interesting question - and one that needs to be examined in its own right.

Rather than the anterior N2 itself being modulated by valence of the distractor, an entirely different explanation is that the pattern of anterior N2 data could be the outcome of the positive deflection of the EPN, riding on top of a cognitive control related anterior N2. The EPN is sometimes accompanied by a positive shift for emotional (compared to neutral) information at fronto-central electrode sites - i.e., a reversal in the polarity of the EPN (see Schupp et al., 2003b, 2006a). Whether the anterior N2 is sensitive to emotion itself, or whether modulation of the anterior N2 by emotion is a byproduct of the frontal deflection of the EPN, will need to be examined in future studies. By directly comparing different factors across components - e.g., the time-course of emotional modulation, magnitude of the modulation by valence, and sensitivity to the same manipulations - it should become clearer whether they index the same, or independent, neural processes ${ }^{7}$. If they reflect the same

\footnotetext{
${ }^{7}$ The current experiment used different reference schemes for the anterior N2 and the EPN. To directly compare components it is ideal to use the same reference scheme. While using different reference schemes does not affect the timing of components, it will likely affect other factors (e.g., the magnitude of emotional modulation) that should be compared to determine whether they reflect the same, or independent, neural processes.
} 
process (i.e., both reflect the EPN - recruitment of attentional resources for the perceptual processing of emotional information) then they should have similar time-courses, similar magnitudes of emotional modulation and be affected similarly by the same manipulations.

\section{Similarities across ERP Components: The Positivity Bias}

One unexpected pattern evident across ERP components in Experiment 2 was that positive images elicited larger neural responses than negative images. This was unexpected as irrelevant positive and negative images (indeed the very same image set used to elicit ERP responses) produce comparable levels of distraction, as demonstrated by a previous study in our lab (Grimshaw et al., in prep), Experiment 1, and Experiment 2 (when data from all participants were included in analyses). The pattern of behavioural data for ERP participants did trend towards producing positive, but not negative, emotional distraction. Thus, it could be that the particular subset of ERP participants was more sensitive to positive, than negative, emotional distractors. If so, then it would be expected that positive images should elicit larger ERPs than negative images.

In a broader context, there has been much evidence for a 'negativity bias' in the literature - that is, negative stimuli affect behavioural responses to a greater extent, and elicit larger neural responses, than positive stimuli (e.g., Carratié, Mercado, Tapia \& Hinojosa, 2001; Ito, Larsen, Smith \& Cacioppo, 1998; Northoff et al., 2000; Peeters \& Czapinsky, 1990; Taylor, 1991). The negativity bias is argued to stem from an evolutionary advantage of responding quickly to threat (Carratié et al., 2001; also see Öhman, 1992). Indeed, responding slowly to pleasurable events has less dire consequences for survival than responding slowly to threat. The larger neural responses to positive, compared to negative, images in Experiment 2 are inconsistent with a negativity bias. However, studies that find evidence for a negativity bias tend to use positive stimuli that are less biologically relevant (and often less arousing) than the negative stimuli used (e.g., images of animals or high adrenaline sports versus images of threat or mutilated bodies; Hajcak et al., 2012). When biologically relevant positive images (e.g., erotic images), of equivalent arousal levels, are used, comparable neural responses for negative and positive stimuli have been reported (e.g., Franken, Muris, Nijs \& Strien 2008; Weinberg \& Hajcak, 2010). The finding that equally arousing positive and negative images elicit comparable neural responses is consistent with the finding that equally arousing positive and negative images produce comparable levels of distraction (Grimshaw et al., in prep.). Interestingly, there is some evidence for a positivity bias when equally arousing positive and negative images are used - that is, positive images have been found to elicit larger neural responses than negative images (e.g., Briggs \& Martin, 
2009; the EPN: Schupp et al., 2004a, 2006b). My findings are consistent with the positivity bias, suggesting that erotic images elicit larger neural responses than images of mutilated bodies, despite images being matched for subjective levels of arousal.

\section{Habituation}

Although I have interpreted the attenuation of ERP responses under high distractor frequency conditions as being caused by proactive control, it is also possible that the attenuation was caused by habituation. While this is an important consideration, it is unlikely with the EPN and LPP. Previous research suggests that the EPN does not habituate to repeated viewing of emotional images (Codispoti, Ferrari \& Bradley, 2007; Codispoti, Ferrari, De Cesarei \& Cardinale, 2006b; Schupp et al., 2006b), and while the overall magnitude of the LPP can attenuate to repeated picture presentations, affective modulation of the LPP persists across repetitions (Codispoti et al., 2006b, 2007; Codispoti, Ferrari \& Bradley, 2006a; Oloffson \& Polich, 2007) These previous studies suggest that habituation is not a likely explanation for the decrease in emotional modulation of the LPP and EPN under high distractor frequency conditions. Further, one pattern in the present data that refutes a habituation account (at least of the LPP) was that positive images modulated the LPP under both low and high distractor frequency conditions. If the attenuation of emotional modulation of ERP components under high distractor frequency was due to habituation, then modulation of the LPP by positive images should have similarly habituated.

It is plausible that the reduction of the anterior N2 under high distractor frequency conditions was due to habituation. Indeed, the anterior N2 has been found to be sensitive to stimulus novelty (see Folstein \& Van Petten, 2008), and decreasing the frequency of a stimulus has been found to elicit larger anterior N2 amplitudes (Nieuwenhuis et al., 2003). To determine whether habituation accounts for the attenuation of the anterior N2 under high distractor frequency conditions (and similarly for the reduction in emotional modulation of the EPN and LPP under high distractor frequency conditions - although a habituation account of the EPN and LPP seems unlikely), future studies could use a manipulation of cognitive control (other than distractor frequency) that keeps the absolute number of distractors presented consistent across conditions - for example, taxing cognitive control resources (De Fockert, Rees, Frith \& Lavie, 2001; Lavie \& De Fockert, 2005; Lavie, Hirst, De Fockert \& Viding, 2004), cueing the location of distractors (Lee \& Chao, 2012; Munneke et al., 2008), or using reward incentives (Locke \& Braver, 2008; Padmala \& Pessoa, 2011, 2014; Pessoa \& Engelmann, 2010). Importantly, if the absolute number of image presentations is matched 
across cognitive control conditions, then any attenuation of the ERPs elicited by distractors in the proactive control condition cannot be attributed to habituation.

\section{Future Directions: Extending our Understanding of how Proactive Mechanisms Control Emotional Distraction}

Finding a Reliable Index of Negative Distractor Processing.

One primary result from Experiment 2 was that the EPN did not reliably index emotional processing of negative distractors. The EPN was chosen as an index of early emotional processing as previous research has reliably observed emotional modulation of this component for both positive and negative distractors, across a range of experimental situations (see Hajcak., 2012; Olofsson et al., 2008; Schupp et al., 2006a). However, it is clear that the EPN was not sensitive to negative images in the current paradigm. To address the question of how proactive mechanisms control negative emotional distraction, future studies could use a different ERP component to index early perceptual processing of negative images. There are a few different early ERP components that could be used to index early perceptual processing of negative images (e.g., the C1 - see Stolarova, Keil \& Moratti, 2006; the N2pc - see Grimshaw et al., 2014; or the P1 - see Santesso et al., 2008). A good candidate component is the $\mathrm{C} 1$. The $\mathrm{C} 1$ is a visual evoked potential (emerging approximately $60-90 \mathrm{~ms}$ after stimulus onset) that is thought to reflect initial processing of the environment in primary visual areas (Di Russo, Matínez \& Hillyard, 2003; Pourtois \& Vuilleumier, 2006; Stolarova et al., 2006). A number of studies have found that the $\mathrm{C} 1$ is enhanced for emotional, compared to neutral, stimuli, and findings are particularly robust for negative stimuli (e.g., Pourtois, Grandjean, Sander \& Vuilleumier, 2004; Rellecke, Palazova, Sommer \& Schacht, 2011; Stolarova et al., 2006). The C1 may reliably index whether early perceptual processing is modulated by emotion allowing a future study to examine the mechanisms by which proactive control reduces negative emotional distraction.

\section{Is Anticipating the Valence or Emotionality of an upcoming Distractor}

\section{Necessary to Engage Proactive Control Mechanisms?}

One question that arises from the current studies is do we need to know that an upcoming distraction is emotional in order to effectively engage proactively mechanisms to control it. Because distractor valence was blocked in my experiments, participants expected distractors in a given block to be of a particular valence. It is not clear from my studies whether effective proactive control of emotional distraction is contingent on knowing the emotionality of distractors. Perhaps we even need to know the valence (i.e., positive or negative) of the upcoming distractor to recruit proactive control processes. It is possible that 
different proactive mechanisms control positive and negative distractions. Indeed, one recent theory (the Asymmetric Inhibition Model; Grimshaw \& Carmel, 2014) suggests that there is a distinction between the mechanisms used to control positive and negative distractors. The left dorsolateral prefrontal cortex (DLPFC; a region known to be involved in cognitive control processes; Kane \& Engle, 2002) is argued to inhibit negative distractions, and the right DLPFC is argued to inhibit positive distractions. To determine whether anticipating the valence (or emotionality) of a distractor is necessary for proactive control strategies to be effective, future studies could use a distraction task that evokes proactive control (e.g., presenting distractors on $75 \%$ of trials) and, critically, manipulate blocking style (distractor valence blocked versus intermixed). Intermixing distractors eliminates the ability to anticipate the valence of an upcoming distractor. If anticipating the valence of distractors is important for effectively using proactive control strategies, then proactive control should only be successfully evoked when distractor valence is blocked, but not intermixed.

Experimentally, this should manifest as increased distraction in the intermixed, compared to the blocked, condition.

\section{Is Proactive Control of Neutral Distraction easier than Proactive Control of}

\section{Emotional Distraction?}

While the current experiments establish that proactive control can effectively suppresses both emotional and neutral distractors, they do not establish whether emotional distractions are as easy to control as neutral distractions. Indeed, due to their potency, controlling attention to emotional distractors may be more effortful, and tax proactive control resources to a greater extent than controlling attention to neutral distractors. One way to determine whether emotional distractions require additional cognitive resources to proactively control would be to examine neural indices of cognitive control. One such index that could be used is alpha activity. Alpha comprises neural oscillations between 8 and 13Hz, and is inversely correlated with task engagement; decreased alpha activity is associated with increased task engagement and focus (Carp \& Compton, 2009; Gould, Rushworth, \& Nobre, 2011; Hanslmayr et al., 2007; Macdonald, Mathan, \& Yeung, 2011). By measuring alpha in an emotional distraction paradigm that evokes proactive control (i.e., a paradigm similar to the current study) it may be possible to determine whether emotional distractions require greater effort to effectively control than neutral distractions. To gauge the extent to which proactive strategies are engaged to control attention to an upcoming distractor, pre-stimulus alpha could be measured in the inter-trial interval - i.e., before the onset of a distractor (Carp $\&$ Compton, 2009). Measuring alpha before the onset of a distractor would ensure that 
anticipatory (i.e., proactive) control processes are being measured. If attention to emotional distractors requires more effort or cognitive resources to proactively control, then decreased alpha activity (i.e., increased cognitive resources allocated to the task) should be observed in blocks of emotional distractors, compared to neutral distractors.

\section{Limitations}

The current studies were designed to assess irrelevant emotional distraction. While distractors were distinct objects, presented in unattended locations, the distractors appeared at the same time as the target letter display. The onset of the letter display (and the distractor) indicated that the trial had begun. It is possible that distractors received greater perceptual prioritisation than they otherwise would have due to the relevance of their onset time (see Gibson \& Kelsey, 1998). In order to make the onset of the distractors irrelevant, the target task itself needs to be free of any dynamic changes (e.g., sudden onsets or movements) that could lead participants to adopt an attentional set for prioritising stimuli with abrupt onsets (Burnham, 2007; Forster \& Lavie, 2011).

One paradigm that could be adapted to examine emotional distraction under conditions that avoid abrupt onsets of targets comes from Forster and Lavie (2011). In this task, a matrix of letters and numbers is presented (and stays on the screen). Participants discriminate whether each item in the matrix is a letter or number (from top to bottom, and left to right, of the array). During the task, irrelevant distractors appear peripherally and their effect on target discrimination at that given time is measured. As the task involves no abrupt onset of targets, the onset of distractors is irrelevant. The task could be adapted to examine irrelevant emotional distraction by simply using emotional and neutral images as distractors. Using a paradigm that presents distractors at irrelevant times would help address the question of whether truly irrelevant emotional distractors are more potent than neutral distractors (as would be expected in a condition that evokes reactive control). Using a distractor frequency manipulation in this irrelevant distraction paradigm would also help determine whether proactive mechanisms can control attention to irrelevant emotional distractors (that have irrelevant onsets) as effectively as irrelevant neutral distractors.

A second primary limitation, that has been mentioned previously, is that the ERP data in Experiment 2 was noisy. The obvious approach to addressing this limitation is to include more trials, and more participants, to reduce noise. This will increase power to detect differences in ERP amplitudes across conditions. However, it is likely that a lot of noise was caused by individual differences. The use of proactive control is flexible and varies across individuals (Braver, 2012). While it is difficult to control for this variation in the data, these 
individual differences are perhaps interesting in their own right. Individual differences in cognitive control ability have been argued to underpin a number of psychopathologies (Braver, 2012; Burgess et al., 2010; Edwards et al., 2010; Eysenck et al., 2007; Mayberg, 1997; West et al., 2010). Braver (2012) even suggests that, rather than a general impairment in cognitive control, different populations may simply have differential tendencies to engage proactive versus reactive control strategies. Indeed, it is plausible that failure to engage proactive control to suppress positive distractions may contribute to acquiring, or maintaining, addiction (see Field \& Cox, 2008). Similarly, failure to engage proactive control to suppress negative distractions may contribute to acquiring, or maintaining, anxiety or depression (see Williams, Mathews \& MacLeod, 1996). Rather than treating individual variation as noise, future research could examine individual differences in cognitive control strategies used to control emotional distraction. This line of research is potentially informative about the role of controlling attention to emotion in mood and addiction disorders.

\section{Conclusions}

In this thesis I examined cognitive control over irrelevant emotional distractions. In Experiment 1, I determined whether proactive mechanisms control emotional distractions as effectively as neutral distractions. When reactive control was evoked, emotional distractors were more potent than neutral distractors. This finding is consistent with studies that have found evidence that entirely irrelevant emotional stimuli are more distracting than irrelevant neutral stimuli (e.g., Fox et al., 2012; Grimshaw et al., in prep.; Yates et al., 2010). Importantly, when proactive control was evoked, emotional images were only marginally more distracting than neutral images. This finding suggests that proactive mechanism control entirely irrelevant emotional distractions almost as effectively as irrelevant neutral distractions, and supports the argument that emotional attention is not independent of topdown control (Pessoa, 2009; Pessoa et al., 2002; Pourtois et al., 2013).

In Experiment 2, ERP responses to emotional and neutral distractors (i.e., images) were measured under proactive and reactive control conditions. The behavioural results of Experiment 2 replicated the pattern of results in Experiment 1 (although the statistical interaction between cognitive control strategy and emotional distraction was not significant when only the data for the subset of ERP participants was analysed). I examined the EPN and LPP to investigate how proactive mechanisms control attention to emotional distractors. If proactive control minimises emotional distraction by preventing early perceptual processing of emotional distractors, then I expected the EPN and LPP to be attenuated (or eliminated) in 
the high distractor frequency condition. Alternatively, if proactive control minimises emotional distraction by preventing sustained attention to, but not early perceptual processing of, emotional distractors, then I expected the LPP to be attenuated (or eliminated) in the high distractor frequency condition, but emotional modulation of the EPN to remain.

Under reactive control conditions positive images elicited a pronounced EPN and LPP (as expected), while negative images only elicited a pronounced LPP. The absence of an EPN to negative images was inconsistent with predictions, but adds to a growing body literature, which suggests that the EPN is particularly sensitive to positive, over negative, information (e.g., Schupp et al., 2004b, 2006a, 2012; Weinberg \& Hajcak, 2010). Importantly, as negative emotional distractors did not modulate the EPN, it could not be used to index early allocation of attentional resources to perceptually process negative distractors in Experiment 2.

Under proactive control conditions, the pronounced EPN to positive images was eliminated. This finding provides support for the hypothesis that proactive mechanisms control positive emotional distraction by reducing early perceptual processing of distractors. However, this interpretation of the data is not clear-cut as positive images elicited a pronounced LPP in the proactive control condition. It is possible that the LPP was elicited independent of active perceptual processing of distractors, or that some perceptual processing of positive distractors still occurred that was not indexed by the EPN. Negative images did not modulate the EPN or LPP under proactive control conditions. The reduction in the LPP suggests that proactive control minimises sustained processing of negative emotional distractors. However, it was not possible to determine whether proactive control minimised negative distraction by preventing early perceptual processing of distractors as the EPN was not a reliable index of negative distractor processing (as indicated by results from the low distractor frequency condition).

Finally, the anterior N2 was examined to determine whether it would effectively index cognitive control strategy used. Indeed, the anterior N2 was more pronounced under low, than high, distractor frequency conditions suggesting that it indexes reactive cognitive control processes.

The research conducted in the current thesis provides evidence that proactive mechanisms can control emotional distraction as effectively as neutral distraction. My research does not clearly provide an answer to the question of how proactive control minimises emotional distraction, but does pave the way for future experiments to address this question. 


\section{References}

Adolphs, R., Tranel, D., Damasio, H., \& Damasio, A. (1994). Impaired recognition of emotion in facial expressions following bilateral damage to the human amygdala. Nature, 372(6507), 669-672. doi:10.1038/372669a0

Adolphs, R., Tranel, D., Damasio, H., \& Damasio, A R. (1995). Fear and the human amygdala. The Journal of Neuroscience: The Official Journal of the Society for Neuroscience, 15(9), 5879-5891. doi:10.1016/j.conb.2008.06.006

American Electroencephalographic Society. (1994). Guidelines for standard electrode position nomenclature. Journal of Clinical Neurophysiology, 11(1), 111-113. Retrieved from http://journals.lww.com/clinicalneurophys/pages/default.aspx

Anderson, A. K., \& Phelps, E. A. (2001). Lesions of the human amygdala impair enhanced perception of emotionally salient events. Nature, 411(6835), 305-309. doi: $10.1038 / 35077083$

Attar, H. C., \& Müller, M. M. (2012). Selective attention to task-irrelevant emotional distractors is unaffected by the perceptual load associated with a foreground task. PloS One, 7(5). doi:10.1371/journal.pone.0037186

Augst, S., Kleinsorge, T., \& Kunde, W. (2014). Can we shield ourselves from task disturbance by emotion-laden stimulation? Cognitive, Affective \& Behavioral Neuroscience, 14(3), 1009-1025. doi:10.3758/s13415-013-0243-x

Barratt, D., \& Bundesen, C. (2012). Attentional capture by emotional faces is contingent on attentional control settings. Cognition \& Emotion, 26(7), 1223-1237. doi:10.1080/02699931.2011.645279

Beck, D. M., \& Lavie, N. (2005). Look here but ignore what you see: Effects of distractors at fixation. Journal of Experimental Psychology: Human Perception and Performance, 31(3), 592-607. doi:10.1037/0096-1523.31.3.592

Berggren, N., Koster, E. H. W., \& Derakshan, N. (2012). The effect of cognitive load in emotional attention and trait anxiety: An eye movement study. Journal of Cognitive Psychology, 24(1), 79-91. doi:10.1080/20445911.2011.618450

Blagrove, E., \& Watson, D. G. (2010). Visual marking and facial affect: Can an emotional face be ignored? Emotion, 10(2), 147-168. doi:10.1037/a0017743

Blagrove, E., \& Watson, D. G. (2014). Ignoring real faces: Effects of valence, threat, and salience. Attention, Perception \& Psychophysics, 76(3), 725-745. doi:10.3758/s13414-013-0572-4 
Botvinick, M. M., Braver, T. S., Barch, D. M., Carter, C. S., \& Cohen, J. D. (2001). Conflict monitoring and cognitive control. Psychological Review, 108(3), 624-652. doi:10.1037//0033-295X.I08.3.624

Bradley, M. M., Codispoti, M., Sabatinelli, D., \& Lang, P. J. (2001). Emotion and motivation II: Sex differences in picture processing. Emotion, 1(3), 300-319. doi:10.1037/15283542.1.3.300

Braver, T. S. (2012). The variable nature of cognitive control: A dual mechanisms framework. Trends in Cognitive Sciences, 16(2), 106-113. doi:10.1016/j.tics.2011.12.010

Braver, T. S., Krug, M. K., Chiew, K. S., Kool, W., Westbrook, J. A., Clement, N. J., . . Somerville, L. H. (2014). Mechanisms of motivation-cognition interaction: Challenges and opportunities. Cognitive, Affective \& Behavioral Neuroscience, 14, 443-72. doi:10.3758/s13415-014-0300-0

Braver, T. S., Paxton, J. L., Locke, H. S., \& Barch, D. M. (2009). Flexible neural mechanisms of cognitive control within human prefrontal cortex. Proceedings of the National Academy of Sciences, 106(18), 7351-7356. doi:10.1073/pnas.0808187106

Breiter, H. C., Etcoff N. L., Whalen P. J., Kennedy W. A., Rauch S. L., Buckner R., L., . . Rosen B, R. (1996). Response and habituation of the human amygdala during visual processing of facial expression. Neuron, 17(5), 875-887. doi:10.1016/S08966273(00)80219-6

Briggs, K. E., \& Martin, F. H. (2009). Affective picture processing and motivational relevance: Arousal and valence effects on ERPs in an oddball task. International Journal of Psychophysiology, 72(3), 299-306. doi:10.1016/j.ijpsycho.2009.01.009

Brosch, T., Pourtois, G., Sander, D., \& Vuilleumier, P. (2011). Additive effects of emotional, endogenous, and exogenous attention: Behavioral and electrophysiological evidence. Neuropsychologia, 49(7), 1779-1787. doi:10.1016/j.neuropsychologia.2011.02.056

Brown, K. W., Goodman, R. J., \& Inzlicht, M. (2012). Dispositional mindfulness and the attenuation of neural responses to emotional stimuli. Social Cognitive and Affective Neuroscience, 8(1), 93-99. doi:10.1093/scan/nss004

Burgess, G. C., \& Braver, T. S. (2010). Neural mechanisms of interference control in working memory: Effects of interference expectancy and fluid intelligence. PLoS ONE, 5(9), 1-11. doi:10.1371/journal.pone.0012861

Burgess, G. C., Depue, B. E., Ruzic, L., Willcutt, E. G., Du, Y. P., \& Banich, M. T. (2010). Attentional control activation relates to working memory in attention- 
deficit/hyperactivity disorder. Biological Psychiatry, 67(7), 632-640.

doi:10.1016/j.biopsych.2009.10.036

Burnham, B. R. (2007). Displaywide visual features associated with a search display's appearance can mediate attentional capture. Psychonomic Bulletin \& Review, 14(3), 392-422. doi:10.3758/BF03194082

Carp, J., \& Compton, R. J. (2009). Alpha power is influenced by performance errors. Psychophysiology, 46(2), 336-343. doi:10.1111/j.1469-8986.2008.00773.x

Carretié, L., Mercado, F., Tapia, M., \& Hinojosa, J. A. (2001). Emotion, attention, and the "negativity bias", studied through event-related potentials. International Journal of Psychophysiology, 41(1), 75-85. doi:10.1016/S0167-8760(00)00195-1

Chao, H. F. (2011). Active inhibition of a distractor word: The distractor precue benefit in the stroop color-naming task. Journal of Experimental Psychology: Human Perception and Performance, 37(3), 799-812. doi:10.1037/a0022191

Clark, L. A., \& Watson, D. (1995). The mini mood and anxiety symptom questionnaire (mini-MASQ). University of Iowa, 1995.

Codispoti, M., Ferrari, V., \& Bradley, M. M. (2006a). Repetitive picture processing: Autonomic and cortical correlates. Brain Research, 1068(1), 213-220. doi:10.1016/j.brainres.2005.11.009

Codispoti, M., Ferrari, V., \& Bradley, M. M. (2007). Repetition and event-related potentials: Distinguishing early and late processes in affective picture perception. Journal of Cognitive Neuroscience, 19(4), 577-586. doi:10.1162/jocn.2007.19.4.577

Codispoti, M., Ferrari, V., De Cesarei, A., \& Cardinale, R. (2006b). Implicit and explicit categorization of natural scenes. Progress in Brain Research, 156, 53-65. doi:10.1016/S0079-6123(06)56003-0

Corbetta, M., \& Shulman, G. L. (2002). Control of goal-directed and stimulus-driven attention in the brain. Nature Reviews Neuroscience, 3(3), 201-215. doi:10.1038/nrn755

Cuthbert, B. N., Schupp, H. T., Bradley, M. M., Birbaumer, N., \& Lang, P. J. (2000). Brain potentials in affective picture processing: Covariation with autonomic arousal and affective report. Biological Psychology, 52(2), 95-111. doi:10.1016/S03010511(99)00044-7

De Cesarei, A., \& Codispoti, M. (2006). When does size not matter? Effects of stimulus size on affective modulation. Psychophysiology, 43(2), 207-215. doi:10.1111/j.14698986.2006.00392.x 
De Fockert, J. W. Rees, G., Frith, C. D., \& Lavie, N (2001). The role of working memory in visual selective attention. Science, 291(5509), 1803-1806. doi:10.1126/science. 1056496

Derryberry, D., \& Reed, M. A. (2002). Anxiety-related attentional biases and their regulation by attentional control. Journal of Abnormal Psychology, 111(2), 225-236. doi:10.1037//0021-843X.111.2.225

Desimone, R., \& Duncan, J. (1995). Neural mechanisms of selective visual attention. Annual Review of Neuroscience, 18(1), 193-222. doi:10.1146/annurev.ne.18.030195.001205

Devue, C., Belopolsky, A. V., \& Theeuwes, J. (2011). The role of fear and expectancies in capture of covert attention by spiders. Emotion, 11(4), 768-775. doi: $10.1037 / \mathrm{a} 0023418$

Di Russo, F., Martinez, A., \& Hillyard, S. A. (2003). Source analysis of event-related cortical activity during visuo-spatial attention. Cerebral Cortex, 13(5), 486-499. doi:10.1093/cercor/13.5.486

Dolcos, F., \& Cabeza, R. (2002). Event-related potentials of emotional memory: Encoding pleasant, unpleasant, and neutral pictures. Cognitive, Affective \& Behavioral Neuroscience, 2(3), 252-263. doi:10.3758/CABN.2.3.252

Dunlap, W. P., Cortina, J. M., Vaslow, J. B., \& Burke, M. J. (1996). Meta-analysis of experiments with matched groups or repeated measures designs. Psychological Methods, 1(2), 170-177. doi:10.1037//1082-989X.1.2.170

Edwards, B. G., Barch, D. M., \& Braver, T. S. (2010). Improving prefrontal cortex function in schizophrenia through focused training of cognitive control. Frontiers in Human Neuroscience, 4, 1-12. doi:10.3389/fnhum.2010.00032

Eimer, M. (1993). Effects of attention and stimulus in a go/nogo task. Biological Psychology, 35(2), 123-138. doi:10.1016/0301-0511(93)90009-W

Eriksen, B. A., \& Eriksen, C. W. (1974). Effects of noise letters upon the identification of a target letter in a nonsearch task. Perception \& Psychophysics, 16(1), 143-149. doi:10.3758/BF03203267

Erthal, F. S., De Oliveira, L., Mocaiber, I., Pereira, M. G., Machado-Pinheiro, W., Volchan, E., \& Pessoa, L. (2005). Load-dependent modulation of affective picture processing. Cognitive, Affective \& Behavioral Neuroscience, 5(4), 388-395. doi:10.3758/CABN.5.4.388 
Eysenck, M. W., Derakshan, N., Santos, R., \& Calvo, M. G. (2007). Anxiety and cognitive performance: attentional control theory. Emotion, 7(2), 336-353. doi:10.1037/15283542.7.2.336

Falkenstein, M., Hoormann, J., \& Hohnsbein, J. (1999). ERP components in go/nogo tasks and their relation to inhibition. Acta Psychologica, 101(2-3), 267-291. doi:10.1016/S0001-6918(99)00008-6

Feinstein, J. S., Goldin, P. R., Stein, M. B., Brown, G. G., \& Paulus, M. P. (2002). Habituation of attentional networks during emotion processing. Neuroreport, 13(10), 1255-1258. doi:10.1097/00001756-200207190-00007

Fernandes, M. A., Koji, S., Dixon, M. J., \& Aquino, J. M. (2011). Changing the focus of attention: The interacting effect of valence and arousal. Visual Cognition, 19(9), 1191-1211. doi:10.1080/13506285.2011.618151

Field, M., \& Cox, W. M. (2008). Attentional bias in addictive behaviors: A review of its development, causes, and consequences. Drug and Alcohol Dependence, 97(1-2), 120. doi:10.1016/j.drugalcdep.2008.03.030

Flaisch, T., Häcker, F., Renner, B., \& Schupp, H. T. (2011). Emotion and the processing of symbolic gestures: An event-related brain potential study. Social Cognitive and Affective Neuroscience, 6(1), 109-118. doi:10.1093/scan/nsq022

Folstein, J. R., \& Van Petten, C. (2008). Influence of cognitive control and mismatch on the N2 component of the ERP: A review. Psychophysiology, 45(1), 152-170. doi:10.1111/j.1469-8986.2007.00602.x

Forster, S., \& Lavie, N. (2008a). Attentional capture by entirely irrelevant distractors, Visual Cognition, 16(2-3), 200-214. doi:10.1080/13506280701465049

Forster, S., \& Lavie, N. (2008b). Failures to ignore entirely irrelevant distractors: The role of load. Journal of Experimental Psychology: Applied, 14(1), 73-83. doi:10.1037/1076898X.14.1.73

Forster, S., \& Lavie, N. (2011). Entirely irrelevant distractors can capture and captivate attention, Psychonomic Bulletin \& Review, 18(6), 1064-1070. doi:10.3758/s13423011-0172-z

Foti, D., Hajcak, G., \& Dien, J. (2009). Differentiating neural responses to emotional pictures: Evidence from temporal-spatial PCA. Psychophysiology, 46(3), 521-530. doi:10.1111/j.1469-8986.2009.00796.x 
Fox, E., Yates, A., \& Ashwin, C. (2012). Trait anxiety and perceptual load as determinants of emotion processing in a fear conditioning paradigm, 12(2), 236-249. doi: $10.1037 / \mathrm{a} 0025321$

Franken, I. H. A., Muris, P., Nijs, I., \& van Strien, J. W. (2008). Processing of pleasant information can be as fast and strong as unpleasant information: Implications for the negativity bias. Netherlands Journal of Psychology, 64(4), 168-176. doi:10.1007/BF03076419

Gibson, B. S., \& Kelsey, E. M. (1998). Stimulus-driven attentional capture is contingent on attentional set for displaywide visual features. Journal of Experimental Psychology: Human Perception and Performance, 24(3), 699-706. doi:10.1037/00961523.24.3.699

Gould, I. C., Rushworth, M. F., \& Nobre, A. C. (2011). Indexing the graded allocation of visuospatial attention using anticipatory alpha oscillations. Journal of Neurophysiology, 105(3), 1318-1326. doi:10.1152/jn.00653.2010

Gratton, G., Coles, M. G. H., \& Donchin, E. (1983). A new method for off-line removal of ocular artifact. Electroencephalography and Clinical Neurophysiology, 55(4), 468484. doi:10.1016/0013-4694(83)90135-9

Grimshaw, G. M., \& Carmel, D. (2014). An asymmetric inhibition model of hemispheric differences in emotional processing. Frontiers in Psychology, 5, 1-7. doi:10.3389/fpsyg.2014.00489

Grimshaw, G. M., Foster, J. J., \& Corballis, P. M. (2014). Frontal and parietal EEG asymmetries interact to predict attentional bias to threat. Brain and Cognition, 90, 7686. doi:10.1016/j.bandc.2014.06.008

Grimshaw, G. M., Kranz, L. S., Moody, R., Carmel, D., Devue, C. (in prep.). Entirely irrelevant emotional distractors are more potent than irrelevant neutral distractors.

Haas, B. W., Omura, K., Constable, R. T., \& Canli, T. (2006). Interference produced by emotional conflict associated with anterior cingulate activation. Cognitive, Affective \& Behavioral Neuroscience, 6(2), 152-156. doi:10.3758/CABN.6.2.152

Haas, B. W., Omura, K., Constable, R. T., \& Canli, T. (2007). Emotional conflict and neuroticism: Personality-dependent activation in the amygdala and subgenual anterior cingulate. Behavioral Neuroscience, 121(2), 249-256. doi:10.1037/07357044.121.6.1173 
Hajcak, G., \& Nieuwenhuis, S. (2006). Reappraisal modulates the electrocortical response to unpleasant pictures. Cognitive, Affective \& Behavioral Neuroscience, 6(4), 291-297. doi:10.3758/CABN.6.4.291

Hajcak, G., \& Olvet, D. M. (2008). The persistence of attention to emotion: Brain potentials during and after picture presentation. Emotion, 8(2), 250-255. doi:10.1037/15283542.8.2.250

Hajcak, G., Weinberg, A., MacNamara, A., \& Foti. (2012) ERPs and the study of emotion. In S. J. Luck \& E. S. Kappenman (Eds.), The Oxford Handbook of Event-Related Potential Components (pp. 441-473). New York: Oxford University Press.

Hanslmayr, S., Aslan, A., Staudigl, T., Klimesch, W., Herrmann, C. S., \& Bäuml, K. H. (2007). Prestimulus oscillations predict visual perception performance between and within subjects. NeuroImage, 37(4), 1465-1473.

doi:10.1016/j.neuroimage.2007.07.011

Henderson, H. A. (2010). Electrophysiological correlates of cognitive control and the regulation of shyness in children. Developmental Neuropsychology, 35(2), 177-193. doi:10.1080/87565640903526538

Herbert, C., Junghofer, M., \& Kissler, J. (2008). Event related potentials to emotional adjectives during reading. Psychophysiology, 45(3), 487-498. doi:10.1111/j.14698986.2007.00638.x

Hodsoll, S., Viding, E., \& Lavie, N. (2011). Attentional capture by irrelevant emotional distractor faces. Emotion, 11(2), 346-353. doi:10.1037/a0022771

Holmes, A., Mogg, K., de Fockert, J., Nielsen, M. K., \& Bradley, B. P. (2014). Electrophysiological evidence for greater attention to threat when cognitive control resources are depleted. Cognitive, Affective \& Behavioral Neuroscience, 14(2), 827835. doi:10.3758/s13415-013-0212-4

Holmes, A., Nielsen, M. K., Tipper, S., \& Green, S. (2009). An electrophysiological investigation into the automaticity of emotional face processing in high versus low trait anxious individuals. Cognitive, Affective \& Behavioral Neuroscience, 9(3), 323334. doi:10.3758/CABN.9.3.323

Hu, K., Padmala, S., \& Pessoa, L. (2013). Interactions between reward and threat during visual processing. Neuropsychologia, 51(9), 1763-1772. doi:10.1016/j.neuropsychologia.2013.05.025 
Ikeda, K., Sugiura, A., \& Hasegawa, T. (2013). Fearful faces grab attention in the absence of late affective cortical responses. Psychophysiology, 50(1), 60-69. doi:10.1111/j.14698986.2012.01478.x

Ito, T. A., Larsen, J. T., Smith, N. K., \& Cacioppo, J. T. (1998). Negative information weighs more heavily on the brain: The negativity bias in evaluative categorizations. Journal of Personality and Social Psychology, 75(4), 887-900. doi:10.1037/00223514.75.4.887

Jodo, E., \& Kayama, Y. (1992). Relation of a negative ERP component to response inhibition in a go/no-go task. Electroencephalography and Clinical Neurophysiology, 82(6), 477-482. doi:10.1016/0013-4694(92)90054-L

Junghöfer, M., Bradley, M. M., Elbert, T. R., \& Lang, P. J. (2001). Fleeting images: A new look at early emotion discrimination. Psychophysiology, 38(2), 175-178. doi:10.1017/S0048577201000762

Kane, M. J., \& Engle, R. W. (2002). The role of prefrontal cortex in working-memory capacity, executive attention, and general fluid intelligence: An individual-differences perspective. Psychonomic Bulletin \& Review, 9(4), 637-671. doi:10.3758/BF03196323

Kleinsorge, T. (2007). Anticipatory modulation of interference induced by unpleasant pictures. Cognition \& Emotion, 21(2), 404-421. doi:10.1080/02699930600625032

Kleinsorge, T. (2009). Anticipation selectively enhances interference exerted by pictures of negative valence. Experimental Psychology, 56(4), 228-235. doi:10.1027/16183169.56.4.228

Koenig, S., \& Mecklinger, A. (2008). Electrophysiological correlates of encoding and retrieving emotional events. Emotion, 8(2), 162-173. doi:10.1037/1528-3542.8.2.162

Kok, A. (1986). Effects of degradation of visual stimuli on components of the event-related potential (ERP) in the go/nogo reaction tasks. Biological Psychology, 23(1), 21-38. doi:10.1016/0301-0511(86)90087-6

Koster, E. H. W., Crombez, G., Verschuere, B., Van Damme, S., \& Wiersema, J. R. (2006). Components of attentional bias to threat in high trait anxiety: Facilitated engagement, impaired disengagement, and attentional avoidance. Behaviour Research and Therapy, 44(12), 1757-1771. doi:10.1016/j.brat.2005.12.011

Kunde, W., \& Mauer, N. (2008). Sequential modulations of valence processing in the emotional stroop task. Experimental Psychology, 55(3), 151-156. doi:10.1027/16183169.55.3.151 
Lang, P. J., Bradley, M. M., \& Cuthbert, B. N. (2008). International affective picture system (IAPS): Affective ratings of pictures and instruction manual. Technical report A-8. Lavie, N. (1995). Perceptual load as a necessary condition for selective attention. Journal of Experimental Psychology: Human Perception and Performance, 21(3), 451-468. doi:10.1037/0096-1523.21.3.451

Lavie, N. (2005). Distracted and confused?: Selective attention under load. Trends in Cognitive Sciences, 9(2), 75-82. doi:10.1016/j.tics.2004.12.004

Lavie, N., \& De Fockert, J. (2005). The role of working memory in attentional capture. Psychonomic Bulletin \& Review, 12(4), 669-674. doi:10.3758/BF03196756

Lavie, N., Hirst, A., De Fockert, J. W., \& Viding, E. (2004). Load theory of selective attention and cognitive control. Journal of Experimental Psychology: General, 133(3), 339-354. doi:10.1037/0096-3445.133.3.339

LeDoux, J. E. (1996). The emotional brain. New York: Simon \& Schuster.

LeDoux, J. E. (2003). Emotion circuits in the brain. In S. Hyman (Ed.), Fear and Anxiety: The Science of Menatal Health (pp. 259-288). New York: Routledge.

Lee, Y. C., \& Chao, H. F. (2012). The role of active inhibitory control in psychological wellbeing and mindfulness. Personality and Individual Differences, 53(5), 618-621. doi:10.1016/j.paid.2012.05.001

Lichtenstein-Vidne, L., Henik, A., \& Safadi, Z. (2012). Task relevance modulates processing of distracting emotional stimuli. Cognition \& Emotion, 26(1), 42-52. doi:10.1080/02699931.2011.567055

Lim, S. L., Padmala, S., \& Pessoa, L. (2008). Affective learning modulates spatial competition during low-load attentional conditions. Neuropsychologia, 46(5),12671278. doi: 10.1016/j.neuropsychologia.2007.12.003

Lipp, O. V, \& Waters, A. M. (2007). When danger lurks in the background: Attentional capture by animal fear-relevant distractors is specific and selectively enhanced by animal fear. Emotion, 7(1), 192-200. doi:10.1037/1528-3542.7.1.192

Locke, H. S., \& Braver, T. S. (2008). Motivational influences on cognitive control: Behavior, brain activation, and individual differences. Cognitive, Affective \& Behavioral Neuroscience, 8(1), 99-112. doi:10.3758/cabn.8.1.99

Luck, S. J. (2005). An introduction to the event-related potential technique. Cambridge, MA: MIT Press. 
Luck, S. J, (2010). Is it legitimate to compare conditions with different numbers of trials? UC-Davis Center for Mind \& Brain, 1-4. Retrieved from http://erpinfo.org/Members/sjluck/Mean_Peak_Noise.pdf

Macdonald, J. S. P., Mathan, S., \& Yeung, N. (2011). Trial-by-trial variations in subjective attentional state are reflected in ongoing prestimulus EEG alpha oscillations. Frontiers in Psychology, 2, 1-16. doi:10.3389/fpsyg.2011.00082

MacLeod, C., Mathews, A, \& Tata, P. (1986). Attentional bias in emotional disorders. Journal of Abnormal Psychology, 95(1), 15-20. doi:10.1037/0021-843X.95.1.15

Mayberg, H. S. (1997). Limbic-cortical dysregulation: a proposed model of depression. The Journal of neuropsychiatry and clinical neurosciences, 9(3), 471-481. doi:10.1176/jnp.9.3.471

McRae, K., Ochsner, K. N., Mauss, I. B., Gabrieli, J. J. D., \& Gross, J. J. (2008). Gender differences in emotion regulation: An fMRI study of cognitive reappraisal. Group Processes \& Intergroup Relations, 11(2), 143-162. doi:10.1177/1368430207088035

Miltner, W. H. R., Krieschel, S., Hecht, H., Trippe, R., \& Weiss, T. (2004). Eye movements and behavioral responses to threatening and nonthreatening stimuli during visual search in phobic and nonphobic subjects. Emotion, 4(4), 323-339. doi:10.1037/15283542.4.4.323

Mogg, K., \& Bradley, B. P. (2005). Attentional bias in generalized anxiety disorder versus depressive disorder. Cognitive Therapy and Research, 29(1), 29-45. doi:10.1007/s10608-005-1646-y

Mogg, K., Bradley, B. P., \& Williams, R. (1995). Attentional bias in anxiety and depression: The role of awareness. The British Journal of Clinical Psychology, 34(1), 17-36. doi:10.1111/j.2044-8260.1995.tb01434.x

Morris, J. S., Friston, K. J., Büchel, C., Frith, C. D., Young, A. W., Calder, A. J., \& Dolan, R. J. (1998). A neuromodulatory role for the human amygdala in processing emotional facial expressions. Brain, 121(1), 47-57. doi:10.1093/brain/121.1.47

Moser, J. S., Hajcak, G., Bukay, E., \& Simons, R. F. (2006). Intentional modulation of emotional responding to unpleasant pictures: An ERP study. Psychophysiology, 43(3), 292-296. doi:10.1111/j.1469-8986.2006.00402.x

Munneke, J., Van der Stigchel, S., \& Theeuwes, J. (2008). Cueing the location of a distractor: An inhibitory mechanism of spatial attention? Acta Psychologica, 129(1), 101-107. doi:10.1016/j.actpsy.2008.05.004 
Nieuwenhuis, S., Yeung, N., \& Cohen, J. D. (2004). Stimulus modality, perceptual overlap, and the go/no-go N2. Psychophysiology, 41(1), 157-160. doi:10.1046/j.14698986.2003.00128.x

Nieuwenhuis, S., Yeung, N., van den Wildenberg, W., \& Ridderinkhof, K. R. (2003). Electrophysiological correlates of anterior cingulate function in a go/no-go task: Effects of response conflict and trial type frequency. Cognitive, Affective \& Behavioral Neuroscience, 3(1), 17-26. doi:10.3758/CABN.3.1.17

Norberg, J., \& Wiens, S. (2013). Effects of attention manipulations on motivated attention to feared and nonfeared negative distracters in spider fear. BMC Neuroscience, 14(1), 113. doi:10.1186/1471-2202-14-139

Northoff, G., Richter, A., Gessner, M., Schlagenhauf, F., Fell, J., Baumgart, F., . . Heinze, H. J. (2000). Functional dissociation between medial and lateral prefrontal cortical spatiotemporal activation in negative and positive emotions: a combined fMRI/MEG study. Cerebral Cortex, 10(1), 93-107. doi:10.1093/cercor/10.1.93

Öhman, A. (1992). Orienting and attention: Preferred preattentive processing of potentially phobic stimuli. In B. A. Campbell, H. Hayne \& R. Richardson (Eds.), Attention and Information Processing in Infants and Adults: Perspectives from Human and Animal Research (pp. 263-295). New Jersey: Lawrence Erlbaum Associates

Okon-Singer, H., Lichtenstein-Vidne, L., \& Cohen, N. (2013). Dynamic modulation of emotional processing. Biological Psychology, 92(3), 480-491. doi:10.1016/j.biopsycho.2012.05.010

Okon-Singer, H., Tzelgov, J., \& Henik, A. (2007). Distinguishing between automaticity and attention in the processing of emotionally significant stimuli. Emotion, 7(1), 147-157. doi:10.1037/1528-3542.7.1.147

Olofsson, J. K., Nordin, S., Sequeira, H., \& Polich, J. (2008). Affective picture processing: An integrative review of ERP findings. Biological Psychology, 77(3), 247-265. doi:10.1016/j.biopsycho.2007.11.006

Olofsson, J. K., \& Polich, J. (2007). Affective visual event-related potentials: Arousal, repetition, and time-on-task. Biological Psychology, 75(1), 101-108. doi:10.1016/j.biopsycho.2006.12.006

Padmala, S., \& Pessoa, L. (2011). Reward reduces conflict by enhancing attentional control and biasing visual cortical processing. Journal of Cognitive Neuroscience, 23(11), 3419-3432. doi:10.1162/jocn_a_00011 
Padmala, S., \& Pessoa, L. (2014). Motivation versus aversive processing during perception. Emotion, 14(3), 450-454. doi:10.1037/a0036112

Pandey, A. K., Kamarajan, C., Tang, Y., Chorlian, D. B., Roopesh, B. N., Manz, N., . . Porjesz, B. (2012). Neurocognitive deficits in male alcoholics: An ERP/sLORETA analysis of the N2 component in an equal probability go/noGo task. Biological Psychology, 89(1), 170-182. doi:10.1016/j.biopsycho.2011.10.009

Peeters, G., \& Czapinski, J. (1990). Positive-negative asymmetry in evaluations: The distinction between affective and informational negativity effects. European Review of Social Psychology, 1(1), 33-60. doi:10.1080/14792779108401856

Pessoa, L. (2009). How do emotion and motivation direct executive control? Trends in Cognitive Sciences, 13(4), 160-166. doi:10.1016/j.tics.2009.01.006

Pessoa, L., \& Engelmann, J. B. (2010). Embedding reward signals into perception and cognition. Frontiers in Neuroscience, 4, 1-8. doi:10.3389/fnins.2010.00017

Pessoa, L., Kastner, S., \& Ungerleider, L. G. (2002). Attentional control of the processing of neural and emotional stimuli. Cognitive Brain Research, 15(1), 31-45. doi:10.1016/S0926-6410(02)00214-8

Pfefferbaum, A., Ford, J. M., Weller, B. J., \& Kopell, B. S. (1985). ERPs to response production and inhibition. Electroencephalography and Clinical Neurophysiology, 60(5), 423-434. doi:10.1016/0013-4694(85)91017-X

Phaf, R. H., \& Kan, K. J. (2007). The automaticity of emotional stroop: A meta-analysis. Journal of Behavior Therapy and Experimental Psychiatry, 38(2), 184-199. doi:10.1016/j.jbtep.2006.10.008

Phan, K. L., Wager, T., Taylor, S. F., \& Liberzon, I. (2002). Functional neuroanatomy of emotion: A meta-analysis of emotion activation studies in PET and fMRI. NeuroImage, 16(2), 331-348. doi:10.1006/nimg.2002.1087

Pourtois, G., Grandjean, D., Sander, D., \& Vuilleumier, P. (2004). Electrophysiological correlates of rapid spatial orienting towards fearful faces. Cerebral Cortex, 14(6), 619-633. doi:10.1093/cercor/bhh023

Pourtois, G., Schettino, A., \& Vuilleumier, P. (2013). Brain mechanisms for emotional influences on perception and attention: What is magic and what is not. Biological Psychology, 92(3), 492-512. doi:10.1016/j.biopsycho.2012.02.007

Pourtois, G., \& Vuilleumier, P. (2006). Dynamics of emotional effects on spatial attention in the human visual cortex. Progress in Brain Research, 156, 67-91. doi:10.1016/S0079-6123(06)56004-2 
Rellecke, J., Palazova, M., Sommer, W., \& Schacht, A. (2011). On the automaticity of emotion processing in words and faces: Event-related brain potentials evidence from a superficial task. Brain and Cognition, 77(1), 23-32. doi:10.1016/j.bandc.2011.07.001

Ridderinkhof, K. R. (2002). Micro- and macro-adjustments of task set: Activation and suppression in conflict tasks. Psychological Research, 66(4), 312-323. doi:10.1007/s00426-002-0104-7

Sander, D., Grafman, J., \& Zalla, T. (2003). The human amygdala: An evolved system for relevance detection. Reviews in the Neurosciences, 14(4), 303-316. doi:10.1515/REVNEURO.2003.14.4.303

Santesso, D. L., Meuret, A. E., Hofmann, S. G., Mueller, E. M., Ratner, K. G., Roesch, E. B., \& Pizzagalli, D. A. (2008). Electrophysiological correlates of spatial orienting towards angry faces: A source localization study. Neuropsychologia, 46(5), 13381348. doi:10.1016/j.neuropsychologia.2007.12.013

Schneider, W., Eschman, A., \& Zuccolotto, A. (2002a). E-prime reference guide. Pittsburgh, PA: Psychology Software Tools.

Schneider, W., Eschman, A., \& Zuccolotto, A. (2002b). E-prime user's guide. Pittsburgh, PA: Psychology Software Tools.

Schupp, H. T., Cuthbert, B. N., Bradley, M. M., Cacioppo, J. T., Ito, T., \& Lang, P. J. (2000). Affective picture processing: The late positive potential is modulated by motivational relevance. Psychophysiology, 37, 257-261. doi:10.1111/1469-8986.3720257

Schupp, H. T., Flaisch, T., Stockburger, J., \& Junghöfer, M. (2006a). Emotion and attention: Event-related brain potential studies. Progress in Brain Research, 156, 31-51. doi:10.1016/S0079-6123(06)56002-9

Schupp, H. T., Junghöfer, M., Weike, A. I., \& Hamm, A. O. (2003a). Attention and emotion: An ERP analysis of facilitated emotional stimulus processing. Neuroreport, 14(8), 1107-1110. doi:10.1097/00001756-200306110-00002

Schupp, H. T., Junghöfer, M., Weike, A. I., \& Hamm, A. O. (2003b). Emotional facilitation of sensory processing in the visual cortex. Psychological Science, 14(1), 7-13. doi:10.1111/1467-9280.01411

Schupp, H. T., Junghöfer, M., Weike, A. I., \& Hamm, A. O. (2004a). The selective processing of briefly presented affective pictures: An ERP analysis. Psychophysiology, 41(3), 441-449. doi:10.1111/j.1469-8986.2004.00174.x 
Schupp, H. T., Ohman, A., Junghöfer, M., Weike, A. I., Stockburger, J., \& Hamm, A. O. (2004b). The facilitated processing of threatening faces: An ERP analysis. Emotion, 4(2), 189-200. doi:10.1037/1528-3542.4.2.189

Schupp, H. T., Schmälzle, R., Flaisch, T., Weike, A. I., \& Hamm, A. O. (2012). Affective picture processing as a function of preceding picture valence: An ERP analysis. Biological Psychology, 92(1), 81-87. doi:10.1016/j.biopsycho.2013.02.006

Schupp, H. T., Stockburger, J., Bublatzky, F., Junghöfer, M., Weike, A. I., \& Hamm, A. O. (2007). Explicit attention interferes with selective emotion processing in human extrastriate cortex. BMC Neuroscience, 8(16). 1-12. doi:10.1186/1471-2202-8-16

Schupp, H. T., Stockburger, J., Codispoti, M., Junghöfer, M., Weike, A. I., \& Hamm, A. O. (2006b). Stimulus novelty and emotion perception: The near absence of habituation in the visual cortex. Neuroreport, 17(4), 365-369.

doi:10.1097/01.wnr.0000203355.88061.c6

Scott, G. G., O’Donnell, P. J., Leuthold, H., \& Sereno, S. C. (2009). Early emotion word processing: Evidence from event-related potentials. Biological Psychology, 80(1), 95104. doi:10.1016/j.biopsycho.2008.03.010

Stolarova, M., Keil, A., \& Moratti, S. (2006). Modulation of the C1 visual event-related component by conditioned stimuli: Evidence for sensory plasticity in early affective perception. Cerebral Cortex, 16(6), 876-887. doi:10.1093/cercor/bhj031

Sussman, T. J., Heller, W., Miller, G. A., \& Mohanty, A. (2013). Emotional Distractors Can Enhance Attention. Psychological Science, 24(11), 2322-2328. doi:10.1177/0956797613492774

Syrjänen, E., \& Wiens, S. (2013). Gender moderates valence effects on the late positive potential to emotional distracters. Neuroscience Letters, 551, 89-93. doi:10.1016/j.neulet.2013.07.018

Taylor, S. E. (1991). Asymmetrical effects of positive and negative events: The mobilizationminimization hypothesis. Psychological Bulletin, 110(1), 67-85. doi:10.1037/00332909.110.1.67

Van den Berg, B., Krebs, R. M., Lorist, M. M., \& Woldorff, M. G. (2014). Utilization of reward-prospect enhances preparatory attention and reduces stimulus conflict. Cognitive, Affective \& Behavioral Neuroscience, 14(2), 561-577. doi:10.3758/s13415-014-0281-z 
Van der Stigchel, S., \& Theeuwes, J. (2006). Our eyes deviate away from a location where a distractor is expected to appear. Experimental Brain Research, 169(3), 338-349. doi:10.1007/s00221-005-0147-2

Viviani, R. (2013). Emotion regulation, attention to emotion, and the ventral attentional network. Frontiers in Human Neuroscience, 7, 1 - 24. doi:10.3389/fnhum.2013.00746

Vuilleumier, P. (2005). How brains beware: Neural mechanisms of emotional attention. Trends in Cognitive Sciences, 9(12), 585-594. doi:10.1016/j.tics.2005.10.011

Vuilleumier, P., Richardson, M. P., Armony, J. L., Driver, J., \& Dolan, R. J. (2004). Distant influences of amygdala lesion on visual cortical activation during emotional face processing. Nature Neuroscience, 7(11), 1271-1278. doi:10.1038/nn1341

Weinberg, A., \& Hajcak, G. (2010). Beyond good and evil: The time-course of neural activity elicited by specific picture content. Emotion, 10(6), 767-782. doi:10.1037/a0020242

West, R., Choi, P., \& Travers, S. (2010). The influence of negative affect on the neural correlates of cognitive control. International Journal of Psychophysiology, 76(2), 107-117. doi:10.1016/j.ijpsycho.2010.03.002

Wiens, S., Molapour, T., Overfeld, J., \& Sand, A. (2012). High negative valence does not protect emotional event-related potentials from spatial inattention and perceptual load. Cognitive, Affective, \& Behavioral Neuroscience, 12(1), 151-160. doi:10.3758/s13415-011-0072-8

Wiens, S., Sand, A., Norberg, J., \& Andersson, P. (2011). Emotional event-related potentials are reduced if negative pictures presented at fixation are unattended. Neuroscience Letters, 495(3), 178-182. doi:10.1016/j.neulet.2011.03.042

Wiens, S., \& Syrjänen, E. (2013). Directed attention reduces processing of emotional distracters irrespective of valence and arousal level. Biological Psychology, 94(1), 4454. doi:10.1016/j.biopsycho.2013.05.001

Willenbockel, V., Sadr, J., Fiset, D., Horne, G. O., Gosselin, F. \& Tanaka, J. W. (2010). Controlling low-level image properties: the SHINE toolbox. Behavior Research Methods, 42(3), 671-684. doi:10.3758/BRM.42.3.671

Williams, J. M. G., Mathews, A., \& MacLeod, C. (1996). The emotional Stroop task and psychopathology. Psychological Bulletin, 120(1), 3-24. doi:10.1037/00332909.120.1.3 
Wronka, E., \& Walentowska, W. (2011). Attention modulates emotional expression processing. Psychophysiology, 48(8), 1047-1056. doi:10.1111/j.14698986.2011.01180.x

Yates, A., Ashwin, C., \& Fox, E. (2010). Does emotion processing require attention? The effects of fear conditioning and perceptual load. Emotion, 10(6), 822-830. doi:10.1037/a0020325

Yiend, J. (2010). The effects of emotion on attention: A review of attentional processing of emotional information. Cognition \& Emotion, 24(1), 3-47.

doi:10.1080/02699930903205698 


\section{Appendix A}

Ratings of Images Used in the Distraction Task

Table A1

Mean (SD) valence and arousal ratings for images used in the distraction task (taken from Lang, Bradley \& Cuthbert, 2008)

\begin{tabular}{|c|c|c|}
\hline & Valence & Arousal \\
\hline $\begin{array}{l}\text { Image } \\
\text { Number }\end{array}$ & Mean & Mean \\
\hline \multicolumn{3}{|l|}{$\underline{\text { Neutral }}$} \\
\hline 2026 & $4.85(0.85)$ & $3.38(1.88)$ \\
\hline 2102 & $5.13(0.99)$ & $2.92(2.02)$ \\
\hline 2221 & $4.33(1.24)$ & $3.05(1.83)$ \\
\hline 2305 & $5.14(0.86)$ & $3.05(1.88)$ \\
\hline 2393 & $4.92(1.05)$ & $2.95(1.95)$ \\
\hline 2397 & $4.93(1.05)$ & $2.56(1.65)$ \\
\hline 2411 & $5.06(0.89)$ & $2.96(1.90)$ \\
\hline 2512 & $4.78(0.94)$ & 3.29 (1.94) \\
\hline 2593 & $5.73(1.50)$ & $3.22(1.96)$ \\
\hline 2595 & $4.97(1.31)$ & 3.65 (1.89) \\
\hline 2745.1 & $5.38(1.22)$ & $3.31(1.95)$ \\
\hline 2840 & $4.90(1.23)$ & $2.55(1.76)$ \\
\hline \multicolumn{3}{|l|}{$\underline{\text { Negative }}$} \\
\hline 3015 & $1.34(0.71)$ & $6.11(2.87)$ \\
\hline 3030 & $1.51(1.07)$ & $7.13(1.88)$ \\
\hline 3059 & $1.47(0.95)$ & $6.50(2.52)$ \\
\hline 3103 & $1.71(1.02)$ & $6.60(2.07)$ \\
\hline 3131 & $1.40(0.84)$ & $6.62(2.30)$ \\
\hline 3140 & $1.50(0.97)$ & $6.94(1.68)$ \\
\hline 3150 & $1.98(1.54)$ & $6.94(2.07)$ \\
\hline 3195 & $1.79(1.06)$ & $6.42(2.53)$ \\
\hline 3550.1 & $1.92(1.34)$ & $6.62(1.90)$ \\
\hline 9253 & $1.60(0.99)$ & $5.65(2.58)$ \\
\hline 9405 & $1.59(1.02)$ & $6.77(2.22)$ \\
\hline
\end{tabular}




\begin{tabular}{rll}
9420 & $1.87(1.54)$ & $6.10(2.37)$ \\
\hline Positive & & \\
4658 & $6.08(2.05)$ & $6.16(2.17)$ \\
4659 & $6.15(2.01)$ & $6.47(2.18)$ \\
4660 & $7.22(1.40)$ & $6.31(1.95)$ \\
4668 & $6.31(1.70)$ & $6.85(1.65)$ \\
4680 & $6.91(1.92)$ & $6.07(2.26)$ \\
4690 & $6.43(1.84)$ & $5.79(2.17)$ \\
4693 & $5.63(1.91)$ & $6.56(1.76)$ \\
4694 & $6.22(1.69)$ & $5.99(2.09)$ \\
4695 & $6.38(1.55)$ & $6.25(2.04)$ \\
4697 & $5.58(1.73)$ & $6.28(1.79)$ \\
4698 & $6.38(1.56)$ & $6.58(1.79)$ \\
4800 & $5.45(2.28)$ & $6.39(1.91)$ \\
\hline
\end{tabular}




\section{Appendix B}

Questionnaire Items for the Mini-MASQ (Clark \& Watson, 1995)

1. Felt really happy (reverse scored)

2. Felt tense or "high strung"

3. Felt depressed

4. Was short of breath

5. Felt withdrawn from other people

6. Felt dizzy or lightheaded

7. Felt hopeless

8. Hands were cold or sweaty

9. Felt like I had a lot to look forward to (reverse scored)

10. Hands were shaky

11. Felt like nothing was very enjoyable

12. Felt keyed up, "on edge"

13. Felt worthless

14. Had trouble swallowing

15. Felt like I had a lot of interesting things to do (reverse scored)

16. Had hot or cold spells

17. Felt like a failure

18. Felt like I was choking

19. Felt really lively, "up" (reverse scored)

20. Felt uneasy

21. Felt discouraged

22. Muscles twitched or trembled

23. Felt like I had a lot of energy

24. Was trembling or shaking

25. Felt like I was having a lot of fun (reverse scored)

26. Had a very dry mouth 


\section{Appendix C}

Questionnaire Items for the Attentional Control Scale (Derryberry \& Reed, 2002)

1. It's very hard for me to concentrate on a difficult task when there are noises around (reverse scored)

2. When I need to concentrate and solve a problem, I have trouble focusing my attention (reverse scored)

3. When I am working hard on something, I still get distracted by events around me (reverse scored)

4. My concentration is good even if there is music in the room around me

5. When concentrating, I can focus my attention so that I become unaware of what's going on in the room around me

6. When I am reading or studying, I am easily distracted if there are people talking in the same room (reverse scored)

7. When trying to focus my attention on something, I have difficulty blocking out distracting thoughts (reverse scored)

8. I have a hard time concentrating when I'm excited about something (reverse scored)

9. When concentrating I ignore feelings of hunger or thirst

10. I can quickly switch from one task to another

11. It takes me a while to get really involved in a new task (reverse scored)

12. It is difficult for me to coordinate my attention between the listening and writing required when taking notes during lectures (reverse scored)

13. I can become interested in a new topic very quickly when I need to

14. It is easy for me to read or write while I'm also talking on the phone

15. I have trouble carrying on two conversations at once (reverse scored)

16. I have a hard time coming up with new ideas quickly (reverse scored)

17. After being interrupted or distracted, I can easily shift my attention back to what I was doing before

18. When a distracting thought comes to mind, it is easy for me to shift my attention away from it

19. It is easy for me to alternate between two different tasks

20. It is hard for me to break from one way of thinking about something and look at it from another point of view (reverse scored) 